\title{
Rhodium-Catalyzed Redox-Neutral Cross- Dehydrogenative Alkenylation of Arylhydrazines for Polymer Synthesis
}

\author{
Benfa Chu, Xuan Wu, Ziwen Fu, Weiping Wu, Bin Wang, and Jin Zhu * \\ Department of Polymer Science and Engineering, School of Chemistry and Chemical \\ Engineering, State Key Laboratory of Coordination Chemistry, Nanjing University, \\ Nanjing 210023, China \\ *Corresponding author. Email: jinz@nju.edu.cn; Phone: +86-25-89686291; Fax:$$
+86-25-83317761
$$

1. General Consideration

2. Materials.

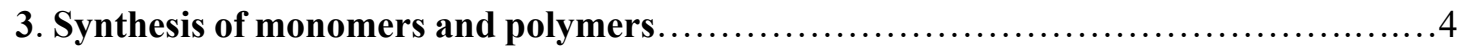

4. Typical polymerization procedure ........................................................

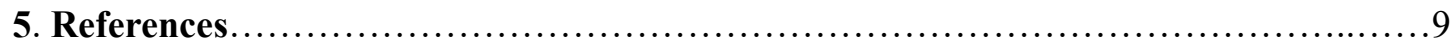

6. Figure S1-S43. Characterization data for monomers and polymers....................10-31

7. Figure S44. Time-dependent UV-vis spectral changes for a DMF solution of P-II,III and

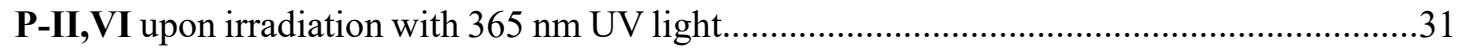

8. Figure S45-S47. Time-dependent UV-vis absorption and emission spectral measured in $\mathrm{CHCl}_{3}$ solution of dimer, P-I and P-II,III .32

9. Figure S48. SEC chromatograms of the polymerization of P-I prepared by using different solvents. .33

10. Table S1. Polymerization of P-I by using $\mathrm{Rh}$ catalyst with different ratios. .33

11. Figure S49. SEC chromatograms of the polymerization of P-I prepared by using Rh catalyst with different ratios. .33

12. Table S2. Polymerization of $\mathbf{P}-\mathbf{I}$ by using additive of HOAc with different ratios. .34

13. Figure S50. SEC chromatograms of the polymerization of P-I prepared by using additive of HOAc with different ratios. .34 
14. Figure S51. SEC chromatograms of the polymerization of P-II,III prepared by using different solvents.

15. Figure S52. SEC chromatograms of the polymer of P-II,III, P-II,IV, P-II,V prepared by using $\mathrm{CH}_{3} \mathrm{OH}$ as solvent.

16. Figure S53. SEC chromatograms of the polymerization of P-II,VI prepared by using different solvents. 35

17. Figure S54. SEC chromatograms of the polymerization of P-II,VII prepared by using different solvents. 35

18. Figure S55. SAXS pattern of P-II,VI upon cooling from $125^{\circ} \mathrm{C}$. .36

19. Figure S56. UV-vis absorption spectra of P-I, P-II,III, P-II,IV and P-II,VI.. .36

20. Figure S57. Fluorescence recovery response of P-I to the addition of different concentrations of EDTA. .36

21. Figure S58. UV-vis absorption spectra of $\boldsymbol{S}$-P-II,VII and PL spectra of spectra of $\boldsymbol{S}$-PII,VII .37

22. Figure S59. TGA trace of P-I with different molecular weight.. .37

23. Figure S60. TGA trace of P-I, P-II,III, P-II,IV, P-II,V P-II,VI, and P-II,VII.. .38 


\section{General Consideration}

Gel permeation chromatography (GPC) data were measured using a Polymer Laboratories PL-GPC 50 instrument equipped with a $5 \mu \mathrm{m}$ guard column, a $5 \mu \mathrm{m}$ mixed-D columns and a refractive index (RI) detector (from Agilent Technology). HPLC grade $N, N$-dimethylformamide (DMF) was used as an eluent at a flow rate of $1 \mathrm{~mL} / \mathrm{min}$. The number-average molecular weight $\left(M_{\mathrm{n}}\right)$ and polydispersity $\left(M_{\mathrm{w}} / M_{\mathrm{n}}\right)$ data are reported relative to polystyrene standards. For the reported $M_{\mathrm{n}}$ in the range of approximately $4 \mathrm{kDa}$ or lower, the value is not considered to be accurate since the GPC trace has already approached the solvent regime. The ${ }^{1} \mathrm{H}$ and ${ }^{13} \mathrm{C}$ NMR spectra were recorded using a Bruker $500 \mathrm{MHz}$ or $600 \mathrm{MHz}$ spectrometer $\{\mathrm{H}\}$. The chemical shifts are expressed in ppm in reference to the residual deuterated solvent and the coupling constants are given in Hz. Data for ${ }^{1} \mathrm{H}$ NMR are recorded as follows: chemical shift (ppm), multiplicity (s, singlet; d, doublet; t, triplet; q, quartet; m, multiplet; br, broad), coupling constant $(\mathrm{Hz})$ and integration. Data for ${ }^{13} \mathrm{C}$ NMR are reported in terms of chemical shift $(\delta$, ppm). Highresolution mass spectrometric data were obtained using Bruker Apex IV RTMS. FT-IR spectra of polymers were recorded on a PerkinElmer Spectrum BX FT-IR system using $\mathrm{KBr}$ pellets. Circular dichroism (CD) spectra were recorded on a JASCO J1500 spectropolarimeter, and the length of the sample cell was $1 \mathrm{~cm}$. Circularly polarized luminescence (CPL) spectra were recorded with a JASCO CPL-300 spectrofluoropolarimeter and the excitation wavelength was $380 \mathrm{~nm}$, scan speed was 50 $\mathrm{nm} / \mathrm{min}$, number of scans was 1 , and slit width was $3000 \mu \mathrm{m}$. UV-vis absorption spectra were measured using Shanghai Metash Instruments UV-8000S UV-Vis-NIR spectrophotometer. Photoluminescence (PL) measurements were carried out on HORIBA Fluolog3 spectrophotometer, and fluorescence lifetime was determined by using HORIBA FL-3 3D fluorescence spectrometer. Thermal gravimetric analysis (TGA) was performed by an SDT851e/SF/1100 ${ }^{\circ} \mathrm{C}$ TGA instrument under nitrogen flow at a heating rate of 10 ${ }^{\circ} \mathrm{C} \min ^{-1}$ from 25 to $800{ }^{\circ} \mathrm{C}$. Differential scanning calorimeter (DSC) was performed on a Q2000 DSC system in nitrogen atmosphere. An indium standard was used for temperature and enthalpy calibrations. All the samples were first heated from 25 to $300{ }^{\circ} \mathrm{C}$ at a rate of $30^{\circ} \mathrm{C} \mathrm{min}-1$ and held at this temperature for $3 \mathrm{~min}$ to eliminate the thermal history, then they were cooled to $25^{\circ} \mathrm{C}$ and heated again from 25 to $300^{\circ} \mathrm{C}$ at a heating or cooling rate of $10^{\circ} \mathrm{C}^{\mathrm{min}^{-1}}$. 


\section{Materials}

All commercial reagents were used without additional purification, unless otherwise stated. Anhydrous solvent was purchased from commercial sources and transferred under argon atmosphere. Analytical thin layer chromatography (TLC) was performed on precoated silica gel 60 GF254 plates. Flash column chromatography was performed using Tsingdao silica gel (60, particle size $0.040-0.063$ $\mathrm{mm})$. Visualization on TLC was achieved by use of UV light $(254 \mathrm{~nm})$ or iodine.

\section{Synthesis of monomers and polymers}

\section{Scheme S1. Synthesis of monomer I}

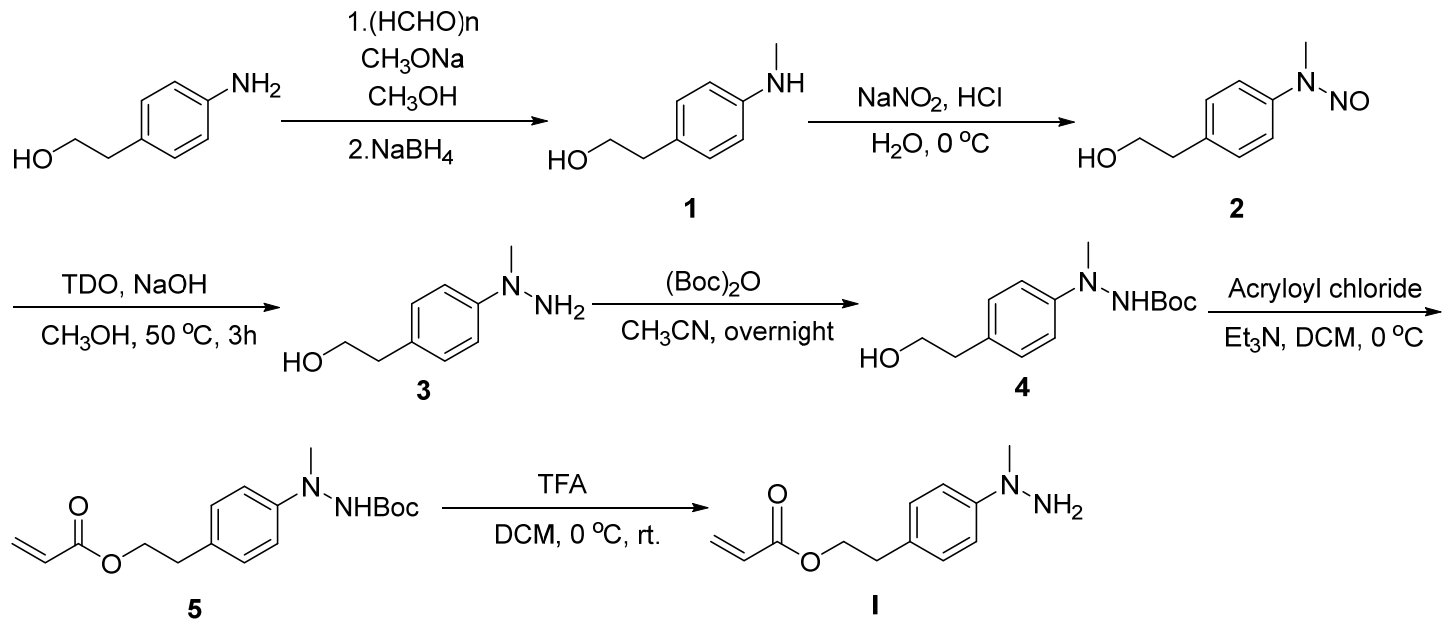

Compound $\mathbf{1}$ and compound $\mathbf{2}$ were prepared according to the reported literatures ${ }^{1}$ and the structures were confirmed by ${ }^{1} \mathrm{H}$ NMR. Compound 1: ${ }^{1} \mathrm{H}$ NMR $(500 \mathrm{MHz}, \mathrm{DMSO}) \delta 6.92(\mathrm{~d}, J=1.2 \mathrm{~Hz} 2 \mathrm{H}, \mathrm{ArH})$, $6.44(\mathrm{~d}, J=2.5 \mathrm{~Hz} 2 \mathrm{H}, \mathrm{ArH}), 5.37(\mathrm{~s}, 1 \mathrm{H}, \mathrm{NH}), 4.53-4.51(\mathrm{~m}, 1 \mathrm{H}, \mathrm{OH}), 3.50-3.48\left(\mathrm{~m}, 2 \mathrm{H}, \mathrm{OCH}_{2}\right)$, $2.63\left(\mathrm{~s}, 3 \mathrm{H}, \mathrm{CH}_{3}\right), 2.57-2.54\left(\mathrm{~m}, 2 \mathrm{H}, \mathrm{CH}_{2}\right) .{ }^{13} \mathrm{C}$ NMR (125 MHz, DMSO) $\delta 148.25,129.31,126.11$, $111.69,62.96,38.48,30.06$. Compound 2: ${ }^{1} \mathrm{H}$ NMR (500 MHz, DMSO) $\delta 7.54(\mathrm{~d}, J=1.2 \mathrm{~Hz} 2 \mathrm{H}, \mathrm{ArH})$, $7.38(\mathrm{~d}, J=2.5 \mathrm{~Hz} 2 \mathrm{H}, \mathrm{ArH}), 4.70(\mathrm{t}, J=3.8 \mathrm{~Hz} 1 \mathrm{H} \mathrm{OH}), 3.65\left(\mathrm{dd}, J=2.5 \mathrm{~Hz} 2 \mathrm{H} \mathrm{OCH}_{2}\right), 3.42(\mathrm{~s}, 3 \mathrm{H}$ $\left.\mathrm{CH}_{3}\right), 2.79\left(\mathrm{t}, J=3.8 \mathrm{~Hz}, 2 \mathrm{H} \mathrm{CH}\right.$ ). ${ }^{13} \mathrm{C}$ NMR $(125 \mathrm{MHz}, \mathrm{DMSO}) \delta 140.12,139.19,129.97,119.52$, $62.05,38.44,31.87$.

Synthesis of $3^{2}: 2(3.6 \mathrm{~g}, 0.02 \mathrm{~mol})$ was allowed to stir in methanol $(2 \mathrm{~mL})$ approximately for $5 \mathrm{~min}$ at $50{ }^{\circ} \mathrm{C}$ to which aqueous solution of sodium hydroxide $(1 \mathrm{M}, 6$ equiv.) followed by thiourea dioxide (TDO,3 equiv.) was added. The reaction was allowed to stir for $3 \mathrm{~h}$ and the progress of the reaction was monitored by TLC. After completion, the reaction mixture was diluted with chloroform and washed with water. The organic layer was dried over anhydrous sodium sulphate, concentrated and subjected for column chromatography (ethyl acetate/hexane $=2: 1$ ) to obtain corresponding pure substituted $\mathbf{3}$ as a yellowish oil. (2.9 g, 87\%). ${ }^{1} \mathrm{H}$ NMR (500 MHz, DMSO) $\delta 6.99$ (d, $\left.J=2.5 \mathrm{~Hz} 2 \mathrm{H}, \mathrm{ArH}\right), 6.88$ (d, $J=1.2$ $\mathrm{Hz} 2 \mathrm{H}, \mathrm{ArH}), 4.55(\mathrm{t}, J=2.5 \mathrm{~Hz} 1 \mathrm{H}, \mathrm{OH}), 4.21\left(\mathrm{~s}, 2 \mathrm{H}, \mathrm{NH}_{2}\right), 3.53-3.49\left(\mathrm{~m}, 2 \mathrm{H}, \mathrm{OCH}_{2}\right), 2.95(\mathrm{~s}, 3 \mathrm{H}$, 
$\left.\mathrm{CH}_{3}\right), 2.61-2.58\left(\mathrm{~m}, 2 \mathrm{H}, \mathrm{CH}_{2}\right) \cdot{ }^{13} \mathrm{C}$ NMR (125 MHz, DMSO) $\delta 151.68,128.92,127.98,114.60,62.83$, 44.23, 37.33. HRMS (ESI) Calcd. for $\mathrm{C}_{9} \mathrm{H}_{15} \mathrm{ON}_{2}:[\mathrm{M}+\mathrm{H}]+$, 167.1179. Found: $\mathrm{m} / \mathrm{z}$ 167.1178.

Synthesis of $4^{3}$ : A mixture of $3(3.36 \mathrm{~g}, 0.02 \mathrm{~mol})$, and dichloromethane $(20 \mathrm{~mL})$ was placed in a round-bottom flask equipped with a magnetic stir bar. The mixture was stirred vigorously, and the temperature was maintained at $0{ }^{\circ} \mathrm{C}$ by the addition of additional ice. To this mixture was added a $15 \mathrm{~mL}$ solution of $(\mathrm{Boc})_{2} \mathrm{O}(2.62 \mathrm{~g}, 0.024 \mathrm{~mol})$ in dichloromethane over the course of $15 \sim 20 \mathrm{~min}$. After stirring overnight at $40{ }^{\circ} \mathrm{C}$, the mixture was concentrated in vacuum and purified by column chromatography (ethyl acetate/hexane $=1: 1$ ) to obtain corresponding pure substituted 4 as a white solid. (4.04 g, 76\%). ${ }^{1} \mathrm{H}$ NMR (500 MHz, DMSO) $\delta 9.06$ (s, $\left.1 \mathrm{H}, \mathrm{NH}\right), 7.02$ (d, $\left.J=2.5 \mathrm{~Hz} 2 \mathrm{H}, \mathrm{ArH}\right), 6.64$ (d, $J=2.5 \mathrm{~Hz} 2 \mathrm{H}$, $\mathrm{ArH}), 4.56(\mathrm{t}, J=2.5 \mathrm{~Hz} 2 \mathrm{H}, \mathrm{OH}), 3.54-3.50\left(\mathrm{~m}, 2 \mathrm{H}, \mathrm{OCH}_{2}\right), 3.02\left(\mathrm{~s}, 3 \mathrm{H}, \mathrm{CH}_{3}\right), 2.60(\mathrm{t}, J=3.8 \mathrm{~Hz} 2 \mathrm{H}$, $\left.\mathrm{CH}_{2}\right), 1.41$ (s, 9H, $\left.\mathrm{C}\left(\mathrm{CH}_{3}\right)_{3}\right){ }^{13} \mathrm{C}$ NMR (125 MHz, DMSO) $\delta 154.95,148.64,129.23,128.93,112.30$, 79.03, 62.72, 40.53, 38.27, 28.24. HRMS (ESI) Calcd. for $\mathrm{C}_{14} \mathrm{H}_{22} \mathrm{O}_{3} \mathrm{~N}_{2} \mathrm{~N}_{\mathrm{a}}$ : [M+Na]+, 289.1523. Found: $\mathrm{m} / \mathrm{z} 289.1521$.

Synthesis of $5^{4}$ : A mixture of $4(2.7 \mathrm{~g}, 0.01 \mathrm{~mol})$ and $\mathrm{Et}_{3} \mathrm{~N}(3.1 \mathrm{~g}, 0.03 \mathrm{~mol})$ were dissolved in dichloromethane $(30 \mathrm{~mL})$, a dichloromethane $(10 \mathrm{~mL})$ solution of acryloyl chloride $(1.35 \mathrm{~g}, 0.015 \mathrm{~mol})$ was added to the solution dropwise at $0^{\circ} \mathrm{C}$. The reaction was monitored by TLC. After completion, the reaction mixture concentrated in vacuum and purified by column chromatography (ethyl acetate/hexane $=1: 1)$ to obtain corresponding pure substituted III as a yellowish oil ${ }^{4}$ ( $\left.2.0 \mathrm{~g}, 63 \%\right) .{ }^{1} \mathrm{H}$ NMR $(500 \mathrm{MHz}$, DMSO) $\delta 9.08$ (s,1H, NH), 7.08 (d, $J=2.5 \mathrm{~Hz} 2 \mathrm{H}, \mathrm{ArH}), 6.67$ (d, $J=2.5 \mathrm{~Hz} 2 \mathrm{H}, \mathrm{ArH}), 6.30$ (d, $J=3.8$ $\left.\mathrm{Hz} 1 \mathrm{H},=\mathrm{CH}_{2}\right), 6.17-6.11(\mathrm{~m}, 1 \mathrm{H},=\mathrm{CH}), 5.92\left(\mathrm{dd}, J=2.5 \mathrm{~Hz} 1 \mathrm{H},=\mathrm{CH}_{2}\right), 4.24\left(\mathrm{t}, J=3.8 \mathrm{~Hz} 2 \mathrm{H}, \mathrm{OCH}_{2}\right)$, $3.03\left(\mathrm{~s}, 3 \mathrm{H}, \mathrm{CH}_{3}\right), 2.81\left(\mathrm{t}, J=3.8 \mathrm{~Hz} 2 \mathrm{H}, \mathrm{CH}_{2}\right), 1.41\left(\mathrm{~s}, 9 \mathrm{H}, \mathrm{C}\left(\mathrm{CH}_{3}\right)_{3}\right) .{ }^{13} \mathrm{C} \mathrm{NMR}(125 \mathrm{MHz}, \mathrm{DMSO}) \delta$ 165.50, 154.96, 148.99, 131.55, 129.29, 128.45,127.20, 112.38, 79.10, 65.08, 40.46, 33.49, 28.23. HRMS (ESI) Calcd. for $\mathrm{C}_{17} \mathrm{H}_{24} \mathrm{O}_{3} \mathrm{~N}_{5} \mathrm{Na}$ : [M+Na]+, 343.1628. Found: m/z 343.1625.

Synthesis of $\boldsymbol{I}^{3}$ : The mixture of $5(3.2 \mathrm{~g} 0.01 \mathrm{~mol})$ and dichloromethane $(20 \mathrm{~mL})$ was stirred vigorously, and the temperature was maintained at $0{ }^{\circ} \mathrm{C}$ by adding ice. The trifluoroacetic acid $(10.0 \mathrm{~mL}$, $0.027 \mathrm{~mol}$ ) was added drop by drop to the reaction mixture stirring overnight at $25^{\circ} \mathrm{C}$. After quenching of the reaction by the addition of saturated aqueous $\mathrm{NaHCO}_{3}$ solution, it was extracted with dichloromethane. The combined organic phase was then washed with brine, dried over $\mathrm{Na}_{2} \mathrm{SO}_{4}$, and evaporated to dryness to give a crude product. This material was further purified by silica gel column chromatography (ethyl acetate/hexane $=1: 1$ ), afforded monomer $\mathbf{I}$ as a yellowish oil $(1.8 \mathrm{~g}, 82 \%) .{ }^{1} \mathrm{H}$ NMR (500 MHz, DMSO) $\delta 7.03$ (d, $J=2.5 \mathrm{~Hz} 2 \mathrm{H}, \mathrm{ArH}), 6.90$ (d, $J=2.5 \mathrm{~Hz} 2 \mathrm{H}, \mathrm{ArH}), 6.30$ (q, $J=3.8$ $\left.\mathrm{Hz} 1 \mathrm{H},=\mathrm{CH}_{2}\right), 6.14(\mathrm{q}, J=2.5 \mathrm{~Hz} 1 \mathrm{H},=\mathrm{CH}), 5.92\left(\mathrm{dd}, J=2.5 \mathrm{~Hz} 1 \mathrm{H},=\mathrm{CH}_{2}\right), 4.26\left(\mathrm{~s}, 2 \mathrm{H}, \mathrm{NH}_{2}\right), 4.22$ (t, $\left.J=3.8 \mathrm{~Hz} 2 \mathrm{H}, \mathrm{OCH}_{2}\right), 2.96$ (s, 3H, CH ), 2.79 (t, $\left.J=3.8 \mathrm{~Hz} 2 \mathrm{H}, \mathrm{CH}_{2}\right) \cdot{ }^{13} \mathrm{C} \mathrm{NMR}(125 \mathrm{MHz}$, DMSO) $\delta$ 165.51, 151.95, 131.57, 128.99, 128.47, 126.12, 113.44, 65.19, 44.02, 33.65, 33.54. HRMS (ESI) Calcd. for $\mathrm{C}_{12} \mathrm{H}_{17} \mathrm{O}_{2} \mathrm{~N}_{2}:[\mathrm{M}+\mathrm{H}]+$, 221.1285. Found: $\mathrm{m} / \mathrm{z} 221.1284$. 
Scheme S2. Synthesis of monomer I-I, IV, Azo-OH, V, VI, and R/S-VII.<smiles>Nc1ccc(Oc2ccc(N)cc2)cc1</smiles>

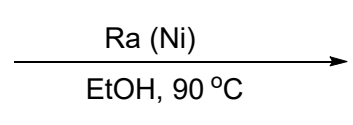<smiles>CCNc1ccc(Oc2ccc(NC(C)(O)[N+](=O)[O-])cc2)cc1</smiles><smiles>CCN(CC)c1ccc(Oc2ccc(N(CC)O[O-])cc2)cc1</smiles>

7

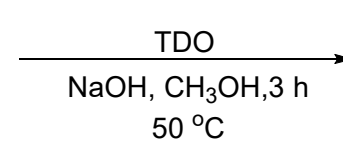<smiles>CCN(N)c1ccc(Oc2ccc(N(N)CC)cc2)cc1</smiles>

II<smiles>C=CC(=O)Oc1ccc(C(C)(C)c2ccc(OC(=O)C=C)cc2)cc1</smiles><smiles>Nc1ccc(O)cc1</smiles><smiles>Cc1ccccc1</smiles><smiles>Oc1ccc(/N=N/c2ccc(O)cc2)cc1</smiles>

Azo-OH<smiles>Oc1ccc(/N=N/c2ccc(O)cc2)cc1</smiles><smiles>CCONCCCCCCCCCCOC</smiles><smiles>C=CC(=O)Oc1ccc(/N=N/c2ccc(OC(=O)C=C)cc2)cc1</smiles><smiles>Oc1ccc2ccccc2c1-c1c(O)ccc2ccccc12</smiles>

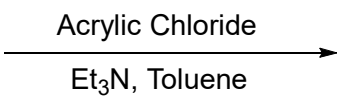

$R / S-B I N O L$<smiles>C=CC(=O)Oc1ccc2ccccc2c1-c1c(OC(=O)C=C)ccc2ccccc12</smiles>

$R / S-V I I$

Synthesis of 6: Three-necked flask fitted with an efficient stirrer, a stopper and a reflux condenser are placed in order, $0.025 \mathrm{~mol}$ of 4,4'-Oxydianiline, $50 \mathrm{~mL}$ of ethanol, about $3 \mathrm{~g}$ of Raney nickel. The mixture is heated under reflux with stirring for a total of 24 hours. then cooled to room temperature and filtered. and purified by phase was evaporated to remove solvent and purified by column chromatography (ethyl acetate/hexane $=4: 1)$ to obtain white flocculent solid, Yield $=89 \% .{ }^{1} \mathrm{H}$ NMR (500 MHz, DMSO) $\delta 6.72-6.68(\mathrm{~m}, 4 \mathrm{H}, \mathrm{ArH}), 6.52-6.49(\mathrm{~m}, 4 \mathrm{H}, \mathrm{ArH}), 5.26(\mathrm{t}, J=2.5 \mathrm{~Hz} 2 \mathrm{H}, \mathrm{NH}), 3.00-2.95(\mathrm{~m}, 4 \mathrm{H}$, $\left.\mathrm{CH}_{2}\right), 1.14\left(\mathrm{t}, J=3.8 \mathrm{~Hz} 6 \mathrm{H}, \mathrm{CH}_{3}\right) .{ }^{13} \mathrm{C}$ NMR $(125 \mathrm{MHz}$, DMSO) $\delta 148.36,144.94,119.10,112.87$, 37.97, 14.56. HRMS (ESI) Calcd. for $\mathrm{C}_{16} \mathrm{H}_{21} \mathrm{ON}_{5}$ : $[\mathrm{M}+\mathrm{H}]+$, 257.1648. Found: $\mathrm{m} / \mathrm{z} 257.1646$.

Compound 7, II were prepared followed the similar procedure described above, and the characterization data are showed below:

Compound 7: 7 as yellow solid, yield $=92 \%,{ }^{1} \mathrm{H}$ NMR (500 MHz, DMSO) $\delta 7.68-7.66(\mathrm{~m}, 4 \mathrm{H}$, $\mathrm{ArH}), 7.25-7.23(\mathrm{~m}, 4 \mathrm{H}, \mathrm{ArH}), 4.06\left(\mathrm{q}, J=2.5 \mathrm{~Hz} 4 \mathrm{H}, \mathrm{CH}_{2}\right), 1.05\left(\mathrm{t}, J=3.8 \mathrm{~Hz} 6 \mathrm{H}, \mathrm{CH}_{3}\right) .{ }^{13} \mathrm{C}^{\mathrm{NMR}}$ (125 MHz, DMSO) $\delta 155.75,136.77,122.23,119.76,39.17,11.46$, HRMS (ESI) Calcd. for $\mathrm{C}_{16} \mathrm{H}_{19} \mathrm{O}_{3} \mathrm{~N}_{4}$ : $[\mathrm{M}+\mathrm{H}]+$, 315.1452. Found: $\mathrm{m} / \mathrm{z} 315.1449$. 
Compound I-I: II as brown solid, yield $=64 \%,{ }^{1} \mathrm{H}$ NMR $(500 \mathrm{MHz}, \mathrm{DMSO}) \delta 6.97-6.94(\mathrm{~m}, 4 \mathrm{H}$, $\mathrm{ArH}), 6.79-6.76(\mathrm{~m}, 4 \mathrm{H}, \mathrm{ArH}), 4.08\left(\mathrm{~s}, 4 \mathrm{H}, \mathrm{NH}_{2}\right), 3.33$ (q, $\left.J=1.2 \mathrm{~Hz} 4 \mathrm{H}, \mathrm{CH}_{2}\right), 1.07$ (t, $J=3.8 \mathrm{~Hz} 6 \mathrm{H}$, $\mathrm{CH}_{3}$ ). ${ }^{13} \mathrm{C}$ NMR (125 MHz, DMSO) $\delta 149.18,147.96,118.64,114.79,49.23,10.13$. HRMS (ESI) Calcd. for $\mathrm{C}_{16} \mathrm{H}_{23} \mathrm{ON}_{4}:[\mathrm{M}+\mathrm{H}]+$, 2 287.1866. Found: $\mathrm{m} / \mathrm{z} 287.1863$.

Compound V: V as white powder, yield $=71 \%,{ }^{1} \mathrm{H}$ NMR (500 MHz, DMSO) $\delta 7.28$ (d, $J=2.5 \mathrm{~Hz}$ 4H, ArH), 7.09 (d, J = 2.5 Hz, ArH), 6.52 (dd, $J=3.8 \mathrm{~Hz} 2 \mathrm{H}, \mathrm{CH}_{2}$ ), $6.42-6.36$ (m, 2H, CH), 6.12 (d, $J$ $\left.=2.5 \mathrm{~Hz} 2 \mathrm{H}, \mathrm{CH}_{2}\right), 1.66\left(\mathrm{~s}, 6 \mathrm{H}, \mathrm{CH}_{3}\right) \cdot{ }^{13} \mathrm{C} \mathrm{NMR}(125 \mathrm{MHz}, \mathrm{DMSO}) \delta 164.25,148.18,147.73,133.57$, $127.78,127.66,121.25,42.10,30.53$.

Compound Azo-OH $\mathbf{\text { : }}$ Azo-OH as black solid, Yield $=76 \%,{ }^{1} \mathrm{H}$ NMR (500 MHz, DMSO): $\delta 10.11$ (s, 2H, OH), 7.72 - 7.70 (m, 4H, ArH), $6.91-6.89(\mathrm{~m}, 4 \mathrm{H}, \mathrm{ArH}) .{ }^{13} \mathrm{C}$ NMR (125 MHz, DMSO) $\delta 160.79$, $146.71,121.47,115.90$.

Monomer $\mathrm{VI}^{6}$ : Azo-OH (2 mmol), $\mathrm{Et}_{3} \mathrm{~N}(2 \mathrm{mmol})$, and acryloyl chloride $(6 \mathrm{mmol})$ were dissolved in dehydrated THF $(100 \mathrm{~mL})$. The solution was mixed at $0^{\circ} \mathrm{C}$ for $4 \mathrm{~h}$ and then reacted at $40^{\circ} \mathrm{C}$ for $24 \mathrm{~h}$. The reaction was stopped by pouring $500 \mathrm{~mL}$ of DI water into the reaction mixture. The solution was then extracted with chloroform several times and dried over $\mathrm{MgSO}_{4}$. The resultant solid was recrystallized in methanol twice to give pure monomer VI (yield: 69\%). ${ }^{1} \mathrm{H}$ NMR (500 MHz, DMSO): $\delta 8.00$ - 7.98 (m, 4H, ArH), 7.46 - 7.44(m, 4H, ArH), 6.60 (dd, $\left.J=5.0 \mathrm{~Hz} 2 \mathrm{H}, \mathrm{CH}_{2}\right), 6.49$ - 6.44 (m, 2H, $\mathrm{CH}), 6.22\left(\mathrm{dd}, J=1.2 \mathrm{~Hz} 2 \mathrm{H}, \mathrm{CH}_{2}\right) .{ }^{13} \mathrm{C}$ NMR $(125 \mathrm{MHz}$, DMSO) $\delta 163.99,152.63,149.68,134.23$, 127.54, 123.99, 123.02. HRMS (ESI) Calcd. for $\mathrm{C}_{18} \mathrm{H}_{15} \mathrm{O}_{4} \mathrm{~N}_{4}:[\mathrm{M}+\mathrm{H}]+$, 323.1026. Found: $\mathrm{m} / \mathrm{z} 323.1024$.

Compound $\boldsymbol{R} / \boldsymbol{S}$-VII ${ }^{6}: \boldsymbol{R} / \boldsymbol{S}$-BINOL ( $\left.2 \mathrm{mmol}\right), \mathrm{Et}_{3} \mathrm{~N}(2 \mathrm{mmol})$, and acryloyl chloride $(6 \mathrm{mmol})$ were dissolved in dehydrated Toluene $(100 \mathrm{~mL})$. The solution was mixed at $0{ }^{\circ} \mathrm{C}$ for $20 \mathrm{~min}$ then reacted at 25 ${ }^{\circ} \mathrm{C}$ for $1 \mathrm{~h}$. The reaction was stopped by pouring $500 \mathrm{~mL}$ of DI water into the reaction mixture. The solution was then extracted with EA several times and dried over $\mathrm{MgSO}_{4}$. The resulting clear solution was concentrated to dryness under reduced pressure and was purified over silica gel column chromatography (PE: DCM/10:1) to afford compound $\boldsymbol{R} / \boldsymbol{S}$-VII $(0.193 \mathrm{~g}, 70 \%$ yield) as a white crystalline solid. ${ }^{1} \mathrm{H}$ NMR $(500 \mathrm{MHz}, \mathrm{CDCl} 3) \delta 8.05$ (4H, dd, $J=1.25$, Ar-H), $7.53-7.45$ (4H, m, ArH), $7.34-7.31(4 \mathrm{H}, \mathrm{m}, \mathrm{Ar}-\mathrm{H}), 6.10\left(2 \mathrm{H}, \mathrm{d}, J=3.75, \mathrm{CH}_{2}\right) .5 .95-5.85(2 \mathrm{H}, \mathrm{m}, \mathrm{CH}), 5.66(2 \mathrm{H}, \mathrm{d}, J=2.5$, $\mathrm{CH}_{2}$ ). $\delta$ 164.33, 146.08, 133.56, 132.47, 131.77, 129.80, 128.28, 127.65, 127.03, 126.38, 125.99, 123.86, 122.93 .

Typical polymerization procedure: A 10-mL sized screw-cap vial with septum and a magnetic bar were dried in oven for $1 \mathrm{~h}$. Under an atmosphere of nitrogen, and loaded with monomers $(0.5 \mathrm{mmol})$, $\left[\mathrm{RhCp}^{*}\left(\mathrm{CH}_{3} \mathrm{CN}\right)_{3}\right]\left(\mathrm{SbF}_{6}\right)_{2}(2.0 \mathrm{~mol} \%), \mathrm{HOAc}(1.2$ eq.). $2 \mathrm{~mL}$ of solvent was injected into the test tube via syringe. The reaction mixtures were then stirred for $12 \mathrm{~h}$ (-AA- and -BB- type monomer stirred for $24 \mathrm{~h})$ at $60^{\circ} \mathrm{C}$. When the reaction reaches the time. The crude residue of polymer was precipitated (P-I: diethyl ether; P-II,VI:CH $\mathrm{CH}_{3} \mathrm{OH}$, and P-II,III, P-II,IV, P-II,V, P-II,VII: hexane) at room temperature; collected by centrifugation, washed with diethyl ether or hexane, and dried under vacuum to afford polymers. The $M_{\mathrm{n}}$ and $M_{\mathrm{w}} / M_{\mathrm{n}}$ of these polymers were characterized by SEC. 
P-I. $86.2 \%$ yield. SEC: $M_{\mathrm{n}}=12.4 \mathrm{kDa}, M_{\mathrm{w}} / M_{\mathrm{n}}=1.39 .{ }^{1} \mathrm{H}$ NMR (500 MHz, $\left.\mathrm{CDCl}_{3}, 25{ }^{\circ} \mathrm{C}\right): \delta 7.81$ - 7.78 (m, ArH), 7.30 - 7.18 (m, ArH), 6.24 - 6.22 (m, CH), 6.37 - 6.33 (m, CH), 4.38 - 4.00 (br, NH and $\left.\mathrm{OCH}_{2}\right), 2.92-2.87\left(\mathrm{~m}, \mathrm{CH}_{2}\right.$ and $\left.\mathrm{CH}_{3}\right) ;{ }^{13} \mathrm{C} \mathrm{NMR}\left(150 \mathrm{MHz}, \mathrm{CDCl}_{3}, 25^{\circ} \mathrm{C}\right): \delta 167.49,146.79,140.51$, 132.54, 128.78, 126.53, 120.08, 118.24, 111.45, 65.49, 34.50, 31.02. Anal. Calcd (\%) for $\mathrm{C}_{12} \mathrm{H}_{13} \mathrm{NO}_{2}: \mathrm{C}$, 70.92; H, 6.45; N, 6.89; Found: C, 69.08; H, 6.58; N, 7.15.

P-II,III. $74.1 \%$ yield. SEC: $M_{\mathrm{n}}=14.9 \mathrm{kDa}, M_{\mathrm{w}} / M_{\mathrm{n}}=1.28 .{ }^{1} \mathrm{H}$ NMR $\left(500 \mathrm{MHz}, \mathrm{CDCl}_{3}, 2{ }^{\circ} \mathrm{C}\right): \delta$ 7.79 - $7.76(\mathrm{~m}, \mathrm{CH}), 7.30$ - $6.82(\mathrm{~m}, \mathrm{ArH}), 6.62-6.59(\mathrm{~m}, \mathrm{ArH}), 6.26-6.22(\mathrm{~m}, \mathrm{CH}), 4.35-3.66(\mathrm{~m}$, $\mathrm{NHCH}_{2}$ and $\left.\mathrm{OCH}_{2}\right), 3.16-3.10(\mathrm{br}, \mathrm{NH}), 1.80-1.10\left(\mathrm{~m}, \mathrm{OCH}_{2}\right.$ and $\left.\mathrm{CH}_{3}\right) .{ }^{13} \mathrm{C} \mathrm{NMR}\left(150 \mathrm{MHz}, \mathrm{CDCl}_{3}\right.$, $\left.25^{\circ} \mathrm{C}\right): \delta 167.45,149.45,143.35,139.94,122.53,120.94,119.92,119.09,118.70,117.93,115.90,114.29$, $113.16,64.32,49.30,44.11,39.25,33.64,29.44,25.70,25.54,15.34,15.13,10.78,10.02$.

P-II,IV. 77.2\% yield. SEC: $M_{\mathrm{n}}=12.9 \mathrm{kDa}, M_{\mathrm{w}} / M_{\mathrm{n}}=1.39 .{ }^{1} \mathrm{H}$ NMR $\left(500 \mathrm{MHz}, \mathrm{CDCl}_{3}, 25{ }^{\circ} \mathrm{C}\right): \delta$ 7.82 - 7.77 (m, CH), 7.07 - 6.93 (m, ArH), 6.42 (br, 1H, CH), 6.29 - 6.25 (br, CH), 4.32 - $4.23(\mathrm{~m}$, $\left.\mathrm{NHCH}_{2}\right), 3.95-3.17$ (m, NH and $\left.\mathrm{OCH}_{2} \underline{\mathrm{CH}_{2}} \mathrm{O}\right), 1.50-0.84\left(\mathrm{~m}, \mathrm{CH}_{3}\right) .{ }^{13} \mathrm{C} \mathrm{NMR}(150 \mathrm{MHz}, \mathrm{CDCl} 3$, $\left.25^{\circ} \mathrm{C}\right): \delta 173.88,171.02,165.85,149.06,143.08,138.99,126.22,120.46,118.76,118.06,112.76,72.45$, $70.50,69.14,63.55,61.60,46.32,43.69,38.87,37.99,33.23,31.49,22.57,14.78,13.25,10.53,9.65$.

P-II,V. $67.7 \%$ yield. SEC: $M_{\mathrm{n}}=10.3 \mathrm{kDa}, M_{\mathrm{w}} / M_{\mathrm{n}}=1.29 .{ }^{1} \mathrm{H}$ NMR $\left(500 \mathrm{MHz}, \mathrm{CDCl}_{3}, 25{ }^{\circ} \mathrm{C}\right): \delta$ 7.96 - $7.93(\mathrm{~m}, \mathrm{CH}), 7.21$ - $6.67(\mathrm{~m}, \mathrm{ArH}), 6.44-6.38(\mathrm{br}, \mathrm{CH}), 3.94-2.72\left(\mathrm{~m}, \mathrm{NH}\right.$ and $\left.\mathrm{CH}_{2} \mathrm{CH}_{3}\right), 1.71$ $1.62\left(\mathrm{~m}, \mathrm{CH}_{2} \mathrm{CH}_{3}\right), 1.30-0.85\left(\mathrm{~m}, 6 \mathrm{H}, \mathrm{C}\left(\mathrm{CH}_{3}\right)_{2}\right) ;{ }^{13} \mathrm{C} \mathrm{NMR}\left(150 \mathrm{MHz}, \mathrm{CDCl}_{3}, 25^{\circ} \mathrm{C}\right): \delta 166.13,164.07$, 155.32, 150.13, 148.82, 147.96, 143.70, 141.08, 128.33, 128.15, 128.08, 121.66, 121.20, 120.69, 120.04, $119.10,117.73,116.61,115.19,114.30,112.91,42.81,42.35,41.87,39.10,33.08,31.28,30.51,30.09$, $29.64,27.53,25.80,23.02,15.17,14.44,12.97,11.55,10.60,9.41,1.34,-0.18$.

P-II,VI. $71 \%$ yield. SEC: $M_{\mathrm{n}}=12.6 \mathrm{kDa}, M_{\mathrm{w}} / M_{\mathrm{n}}=1.26 .{ }^{1} \mathrm{H}$ NMR $\left(500 \mathrm{MHz}, \mathrm{CDCl}_{3}, 25{ }^{\circ} \mathrm{C}\right): \delta$ 7.93 - 7.72 (m, ArH and $\mathrm{CH}), 7.29$ - $6.68(\mathrm{~m}, \mathrm{ArH}), 6.47-6.44(\mathrm{~m}, \mathrm{CH}), 3.85-2.82\left(\mathrm{~m}, \mathrm{NH}\right.$ and $\mathrm{CH}_{2}$ ), 1.29 - $0.84\left(\mathrm{~m}, \mathrm{CH}_{3}\right) .{ }^{13} \mathrm{C} \mathrm{NMR}\left(150 \mathrm{MHz}, \mathrm{CDCl}_{3}, 2{ }^{\circ} \mathrm{C}\right): \delta 171.22,165.44,152.92,152.67,151.98$, $150.81,150.39,150.13,149.25,146.62,145.92,145.77,143.44,141.91,124.98,124.46,124.13,124.10$, $124.03,123.70,123.67,123.42,122.58,122.33,122.24,122.19,122.10,120.19,119.77,119.45,119.28$, $119.18,119.09,119.01,118.03,116.97,116.87,116.71,116.02,115.91,113.21,77.27,46.60,46.43$, $43.90,41.20,39.03,38.81,33.76,15.08,14.87,11.28,10.38,9.79,8.74,8.61,0.03$.

P-II,VII. 69.3\%. yield. SEC: $M_{\mathrm{n}}=7.2 \mathrm{kDa}, M_{\mathrm{w}} / M_{\mathrm{n}}=1.47 .{ }^{1} \mathrm{H} \mathrm{NMR}\left(500 \mathrm{MHz}, \mathrm{CDCl}_{3}, 25{ }^{\circ} \mathrm{C}\right)$ : $\delta 7.93-7.78$ (m, ArH and $\mathrm{CH}), 7.47-6.63(\mathrm{~m}, \mathrm{ArH}$ and $\mathrm{CH}), 4.25-3.7$ (m, $\mathrm{NH}$ and $\left.\mathrm{CH}_{2}\right), 1.44-0.89$ (m, $\left.\mathrm{CH}_{3}\right) .{ }^{13} \mathrm{C}$ NMR $\left(150 \mathrm{MHz}, \mathrm{CDCl}_{3}, 25{ }^{\circ} \mathrm{C}\right): \delta 167.20,165.49,152.03,149.11,148.28,147.14$, $143.59,141.24,133.87,133.60,132.38,131.71,130.48,129.75,128.31,127.09,126.35,126.14,124.92$, $123.79,123.22,122.66,122.19,120.12,118.65,116.94,116.00,115.39,114.41,114.02,112.94,49.66$, $47.09,43.71,39.05,34.67,33.48,32.69,29.97,15.33,15.05,12.94,11.80,11.21,8.96,0.29$. 


\section{References}

(1) Liu, Z. S.; Zeng, H.; Zhang, W, J.; Song, C.; Yang, F.; Liu, Y.; Zhu, J. Rh(III)-catalyzed N-nitrosodirected C-H Olefination Polymerization. Polymer 2019,172, 152-159.

(2) Chaudharya, P.; Guptaa, S.; Sureshbabub, P.; Sabiah, S.; Kandasamy, J. A Metal Free Reduction of aryl- $N$-nitrosamines to Corresponding Hydrazines Using Sustainable Reductant Thiourea Dioxide. Green Chem., 2016,18, 6215-6221.

(3) Zhou, S. G.; Wang, J. H.; Zhang, F. F.; Song, C.; Zhu. J. A Versatile, Traceless C-H Activation-Based Approach for the Synthesis of Heterocycles. Org. Lett. 2016, 18(10), 2427-2430.

(4) Zhang, B. H.; Lv, X. Q.; Zhu, A. Q.; Zheng, J. W.; Yang, Y. Q.; An, Z. S. Morphological Stabilization of Block Copolymer Worms Using Asymmetric Cross-Linkers during Polymerization-Induced SelfAssembly. Macromolecules 2018, 51(8), 2776-2784.

(5) Niyomsin, S.; Chirachanchai, S. Poly(acrylic acid) with Benzoxazine-Based Supramolecular Crosslinker for Responsive and Reversible Functional Hydrogel. European Polymer Journal 2018, 105 , 451-458.

(6) Li, C. Z.; Moon, J.; Yun, G-H.; Kim, H.; Cho. M. Influence of External Loads on Structure and Photoactuation in Densely Crosslinked Azo-Incorporated Liquid Crystalline Polymers. Polymer 2017, $129,252-260$. 


\section{Figure S1-S43 Characterization data for monomers and polymers}
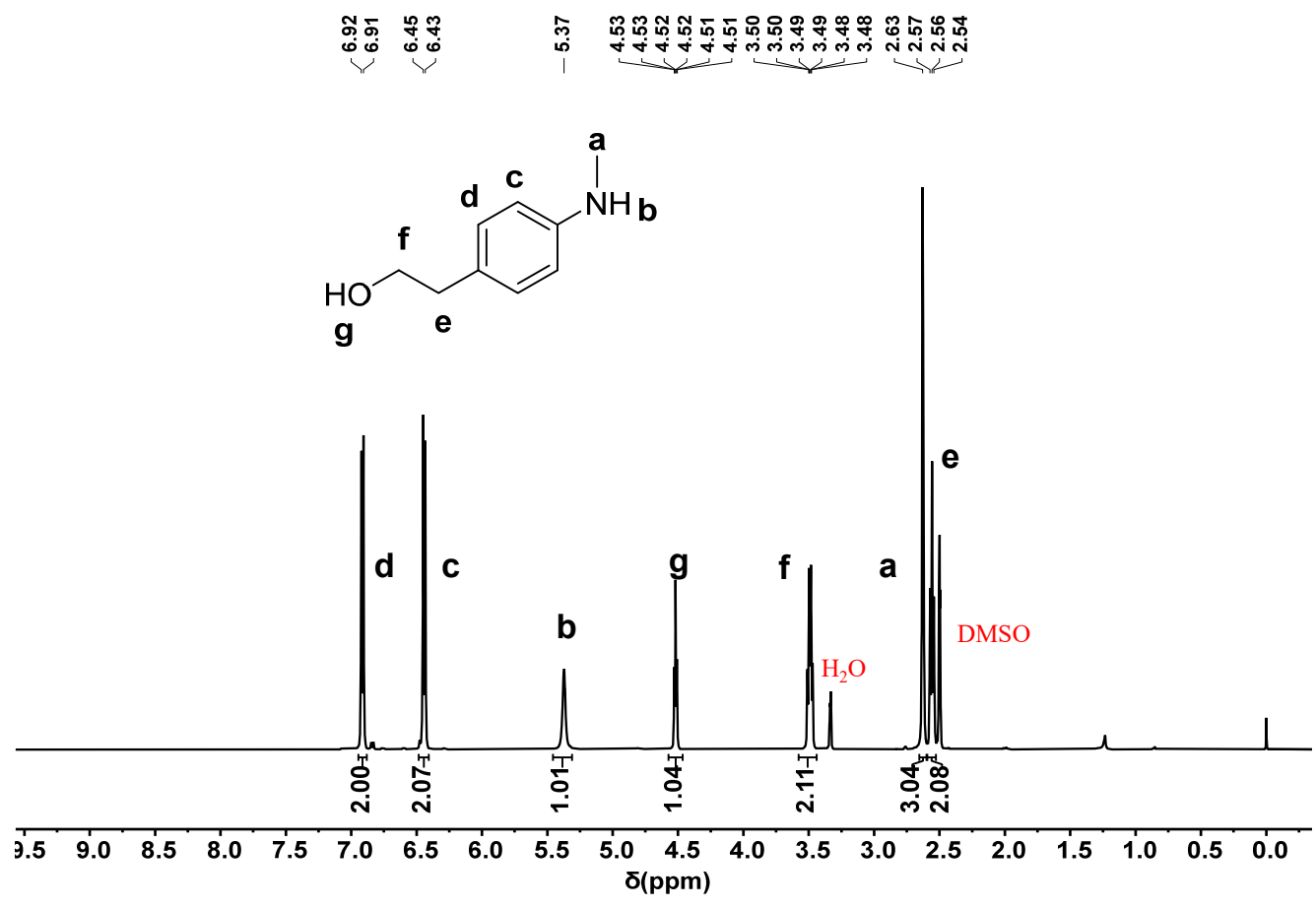

Figure S1. ${ }^{1} \mathrm{H}$ NMR $(500 \mathrm{MHz})$ spectrum of Compound $\mathbf{1}$ measured in DMSO at $25^{\circ} \mathrm{C}$.

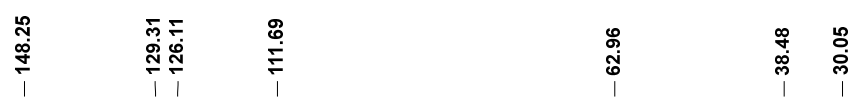

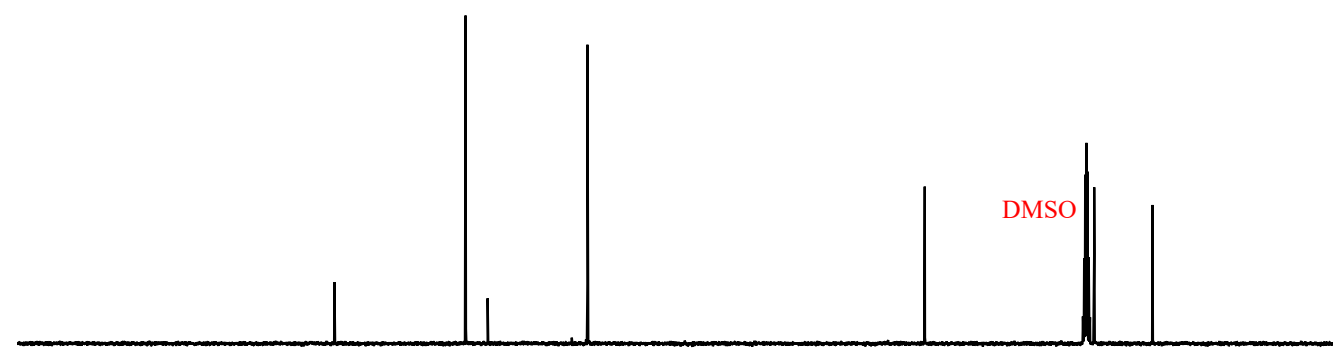

$\begin{array}{lllllllllllllllllll}190 & 180 & 170 & 160 & 150 & 140 & 130 & 120 & 110 & \begin{array}{c}100 \\ \delta(\mathrm{ppm})\end{array} & 90 & 80 & 70 & 60 & 50 & 40 & 30 & 20 & 10\end{array}$

Figure S2. ${ }^{13} \mathrm{C}$ NMR $(125 \mathrm{MHz})$ spectrum of Compound $\mathbf{1}$ measured in DMSO at $25{ }^{\circ} \mathrm{C}$. 
<smiles>CN(C)c1ccc(CCOP)cc1</smiles>

a

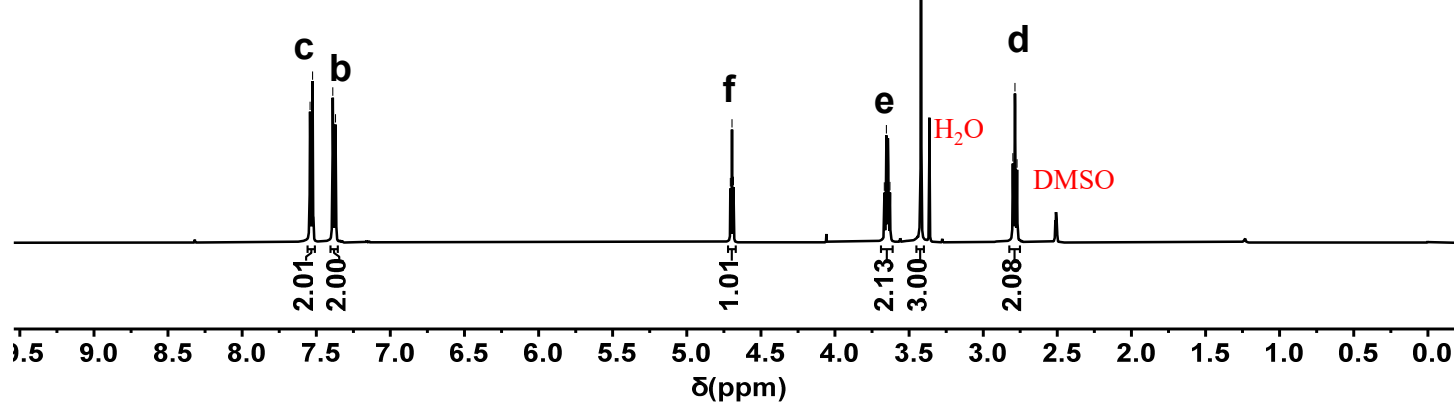

Figure S3. ${ }^{1} \mathrm{H}$ NMR $(500 \mathrm{MHz})$ spectrum of Compound 2 measured in DMSO at $25^{\circ} \mathrm{C}$.
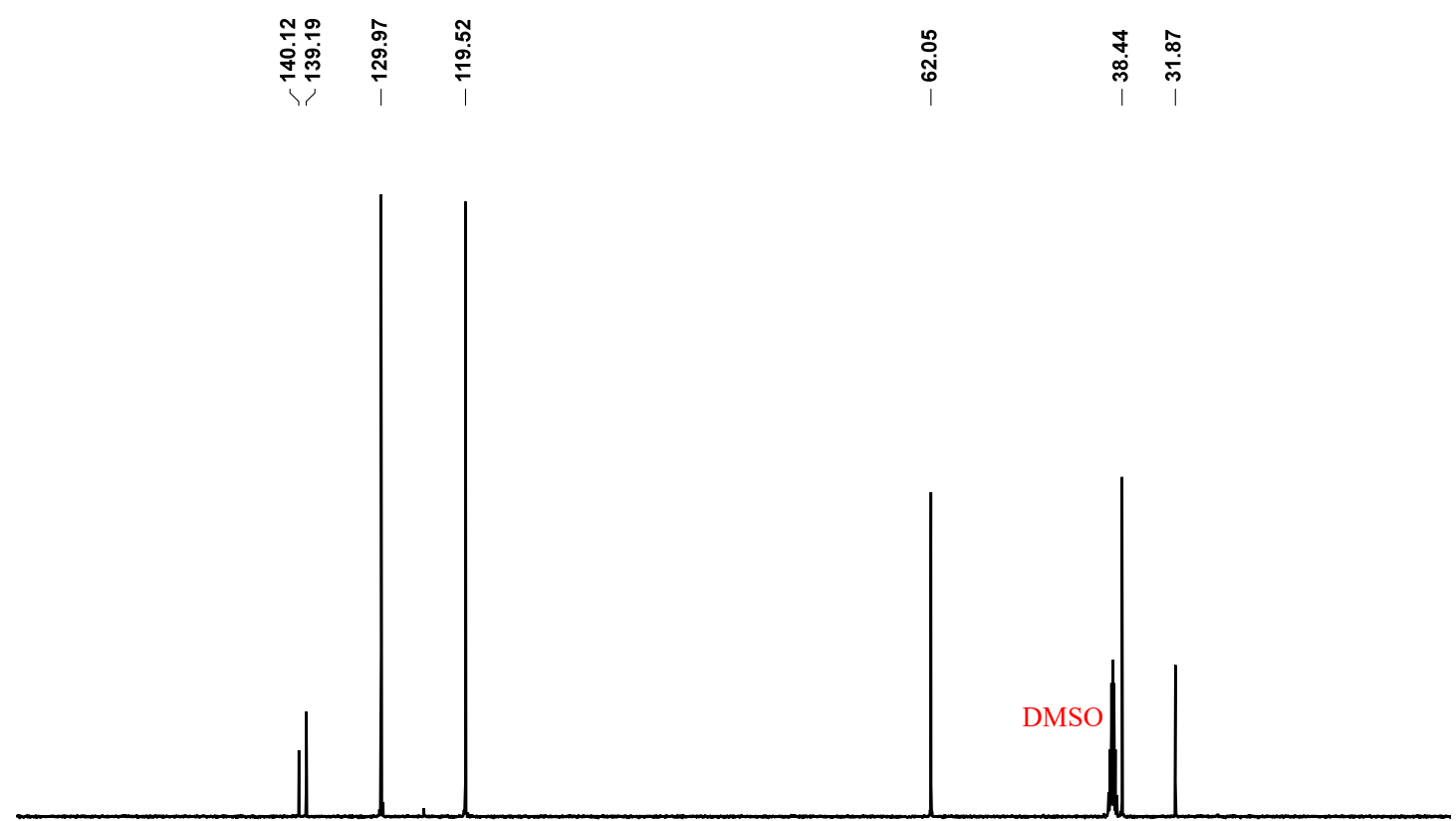

$\begin{array}{llllllllllllllllll}170 & 160 & 150 & 140 & 130 & 120 & 110 & 100 & 90 & 80 & 70 & 60 & 50 & 40 & 30 & 20 & 10 & 0\end{array}$

Figure S4. ${ }^{13} \mathrm{C}$ NMR $(125 \mathrm{MHz})$ spectrum of Compound 2 measured in DMSO at $25{ }^{\circ} \mathrm{C}$. 
<smiles>CC(O)Cc1ccc(N(C)N)cc1</smiles>

a

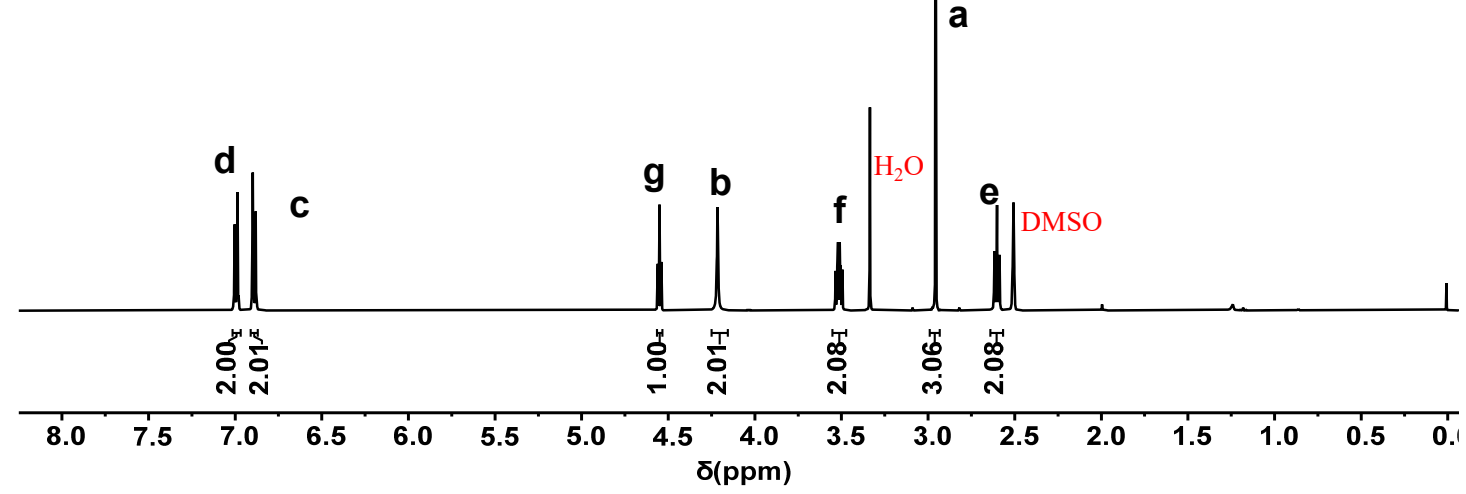

Figure S5. ${ }^{1} \mathrm{H}$ NMR $(500 \mathrm{MHz})$ spectrum of Compound 3 measured in DMSO at $25^{\circ} \mathrm{C}$.
$\stackrel{\infty}{\stackrel{0}{5}}$
i্ৰ:

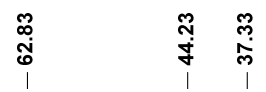

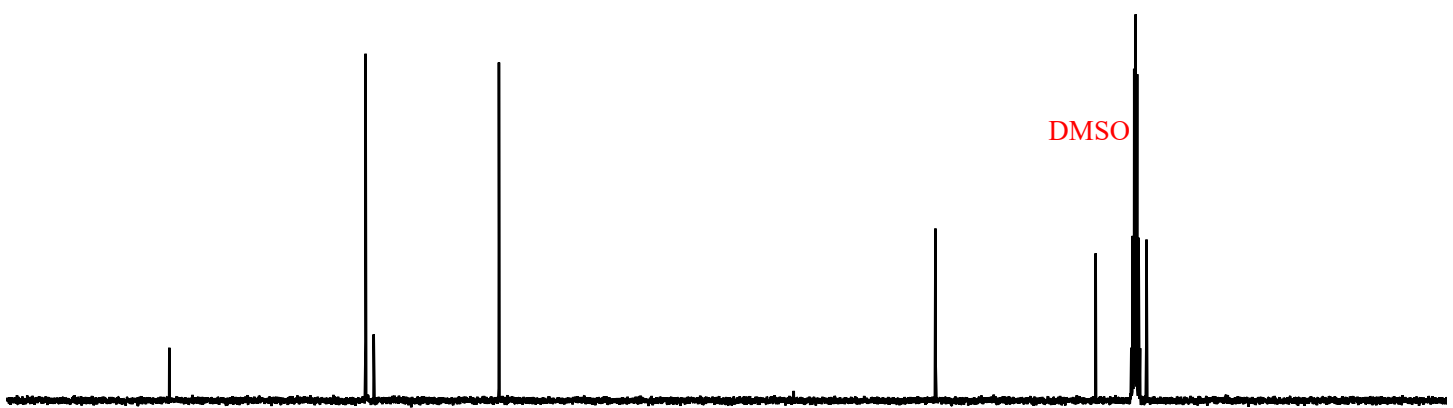

\begin{tabular}{lllllllllllllllll}
\hline 70 & 160 & 150 & 140 & 130 & 120 & 110 & 100 & 90 & 80 & 70 & 60 & 50 & 40 & 30 & 20 & 10
\end{tabular}

Figure S6. ${ }^{13} \mathrm{C}$ NMR $(125 \mathrm{MHz})$ spectrum of Compound 3 measured in DMSO at $25{ }^{\circ} \mathrm{C}$. 
<smiles>CC(CO)c1ccc(N(C)NC(=O)OC(C)(C)C)cc1</smiles>

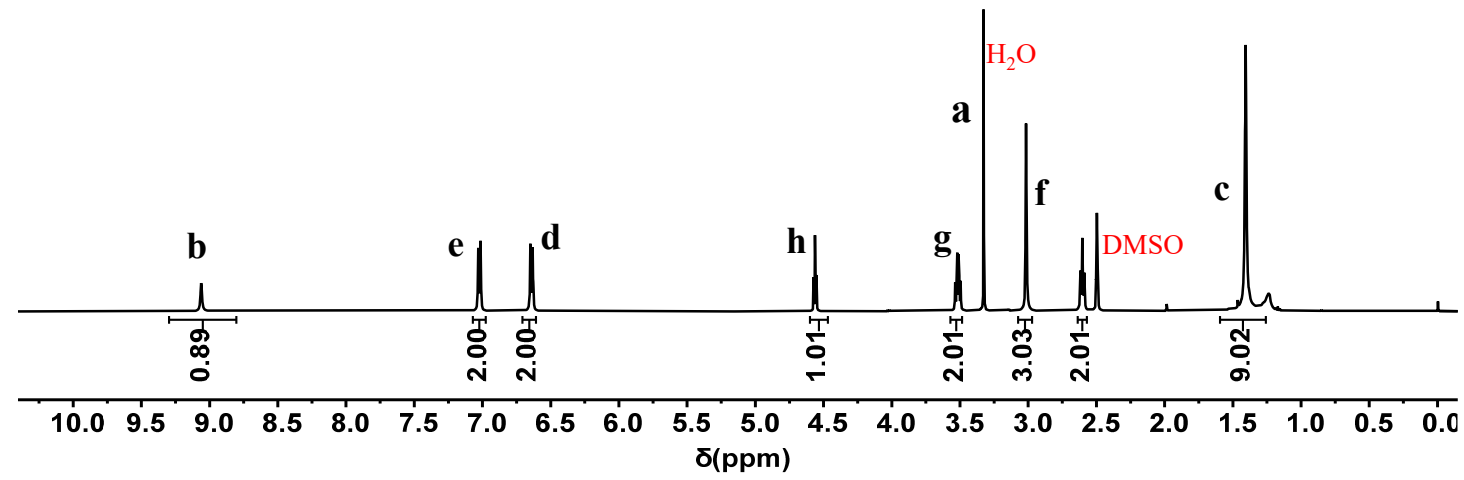

Figure S7. ${ }^{1} \mathrm{H}$ NMR $(500 \mathrm{MHz})$ spectrum of Compound 4 measured in DMSO at $25^{\circ} \mathrm{C}$.

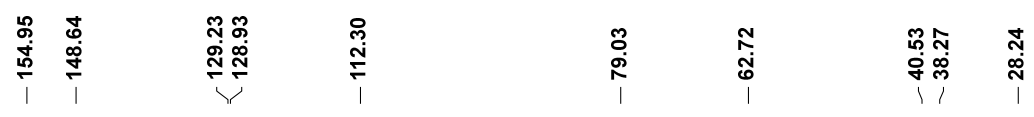

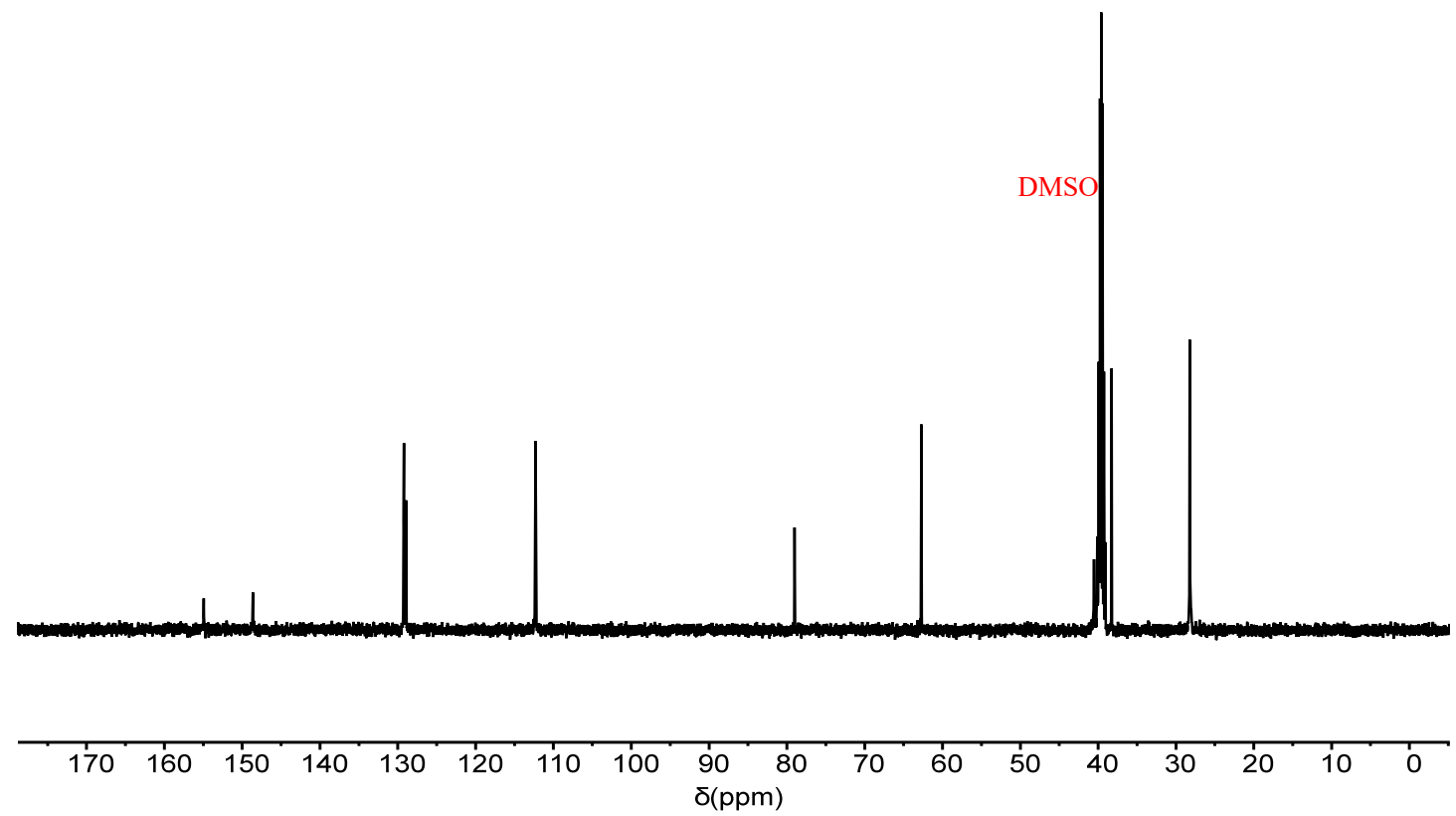

Figure S8. ${ }^{13} \mathrm{C}$ NMR $(125 \mathrm{MHz})$ spectrum of Compound 4 measured in DMSO at $25{ }^{\circ} \mathrm{C}$. 
<smiles>CC(C)(C)OC(=O)NN([10IH])c1ccc(C(I)COC(=O)/C=C\I)cc1</smiles><smiles>CCO</smiles>

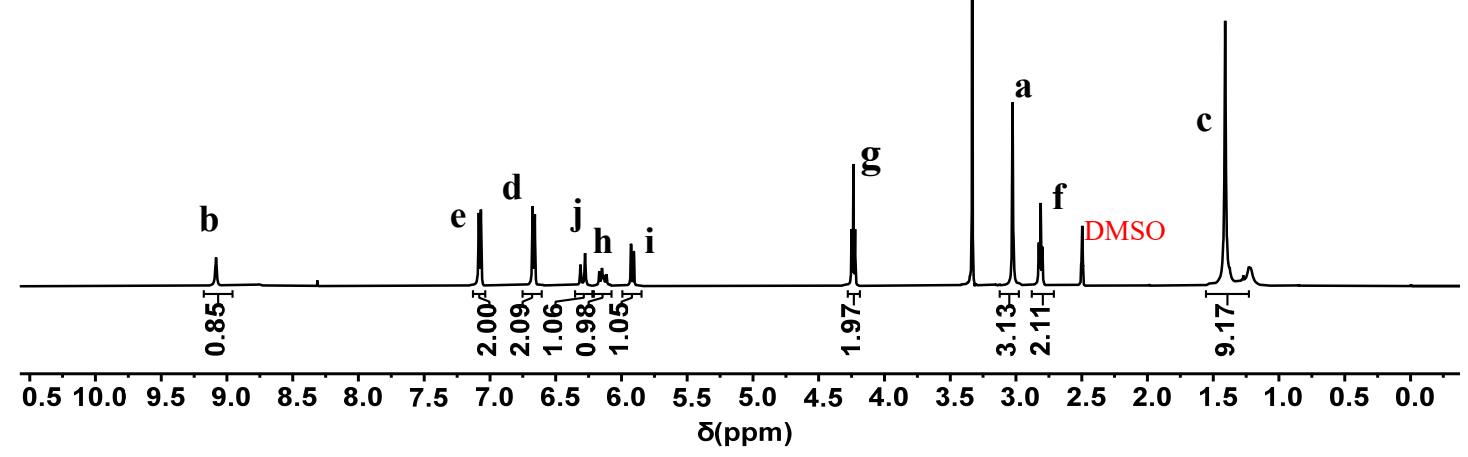

Figure S9. ${ }^{1} \mathrm{H}$ NMR $(500 \mathrm{MHz})$ spectrum of Compound 5 measured in DMSO at $25^{\circ} \mathrm{C}$.

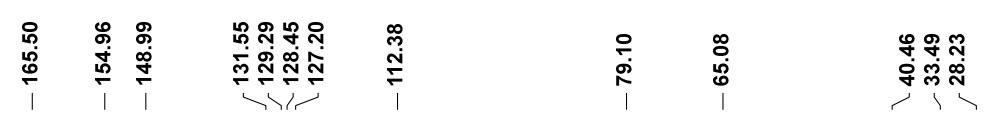

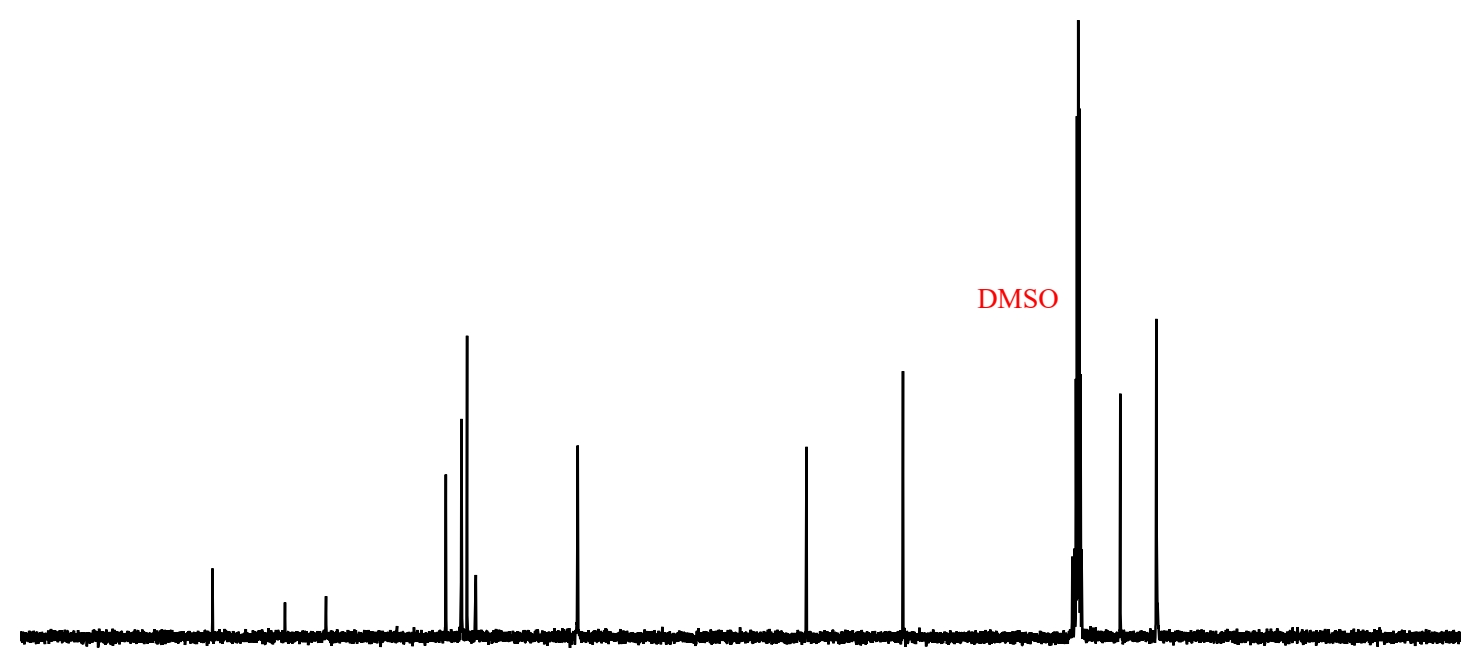

$\begin{array}{rllllllllllllllllllll}190 & 180 & 170 & 160 & 150 & 140 & 130 & 120 & 110 & 100 & \begin{array}{c}90 \\ \delta(\mathrm{ppm})\end{array} & 80 & 70 & 60 & 50 & 40 & 30 & 20 & 10 & 0 & -10\end{array}$

Figure S10. ${ }^{13} \mathrm{C}$ NMR $(125 \mathrm{MHz})$ spectrum of Compound 5 measured in DMSO at $25{ }^{\circ} \mathrm{C}$. 

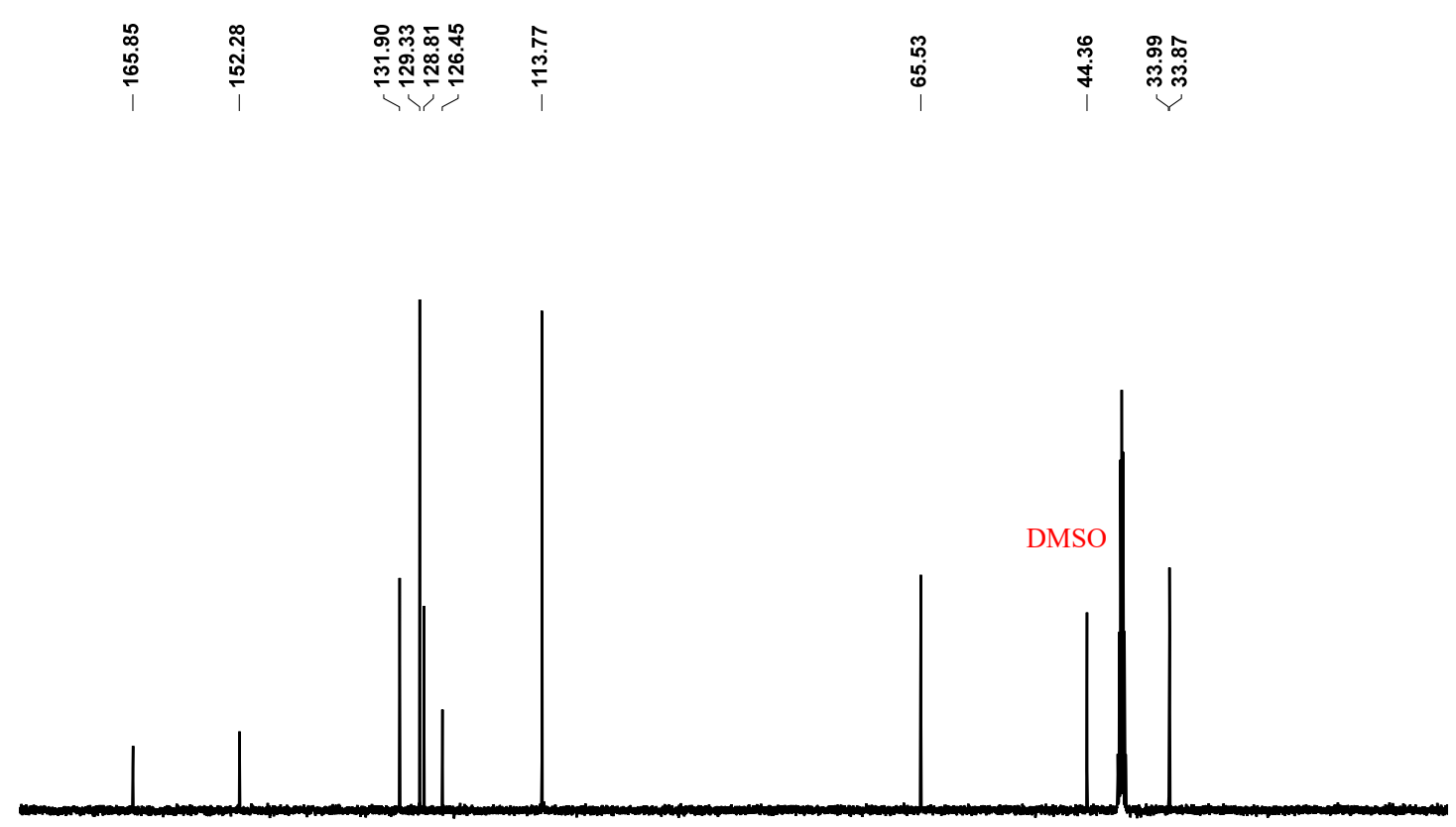

\begin{tabular}{lllllllllllllllllll}
\hline 80 & 170 & 160 & 150 & 140 & 130 & 120 & 110 & 100 & $\begin{array}{c}90 \\
\delta(\mathrm{ppm})\end{array}$ & 80 & 70 & 60 & 50 & 40 & 30 & 20 & 10 & 0
\end{tabular}

Figure S11 ${ }^{13} \mathrm{C}$ NMR $(125 \mathrm{MHz})$ spectrum of monomer I measured in DMSO at $25{ }^{\circ} \mathrm{C}$.

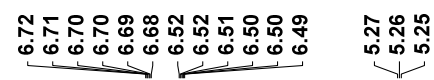

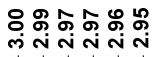

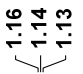<smiles>CCNc1ccc(Oc2ccc(NCC)cc2)cc1</smiles>

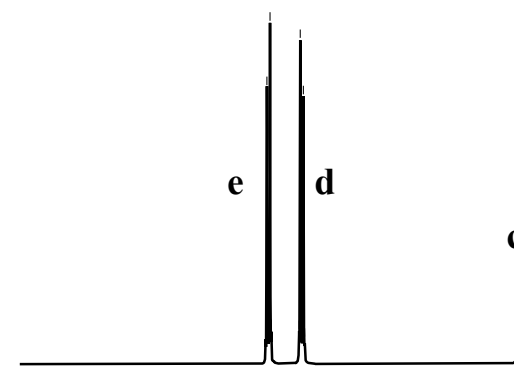

a

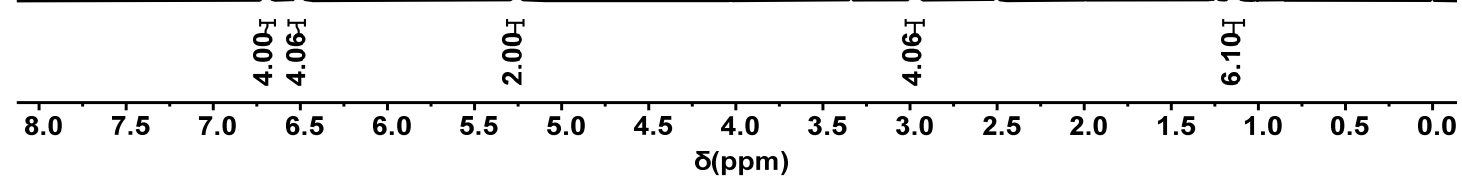

Figure S12. ${ }^{1} \mathrm{H}$ NMR $(500 \mathrm{MHz})$ spectrum of Compound 6 measured in DMSO at $25^{\circ} \mathrm{C}$. 

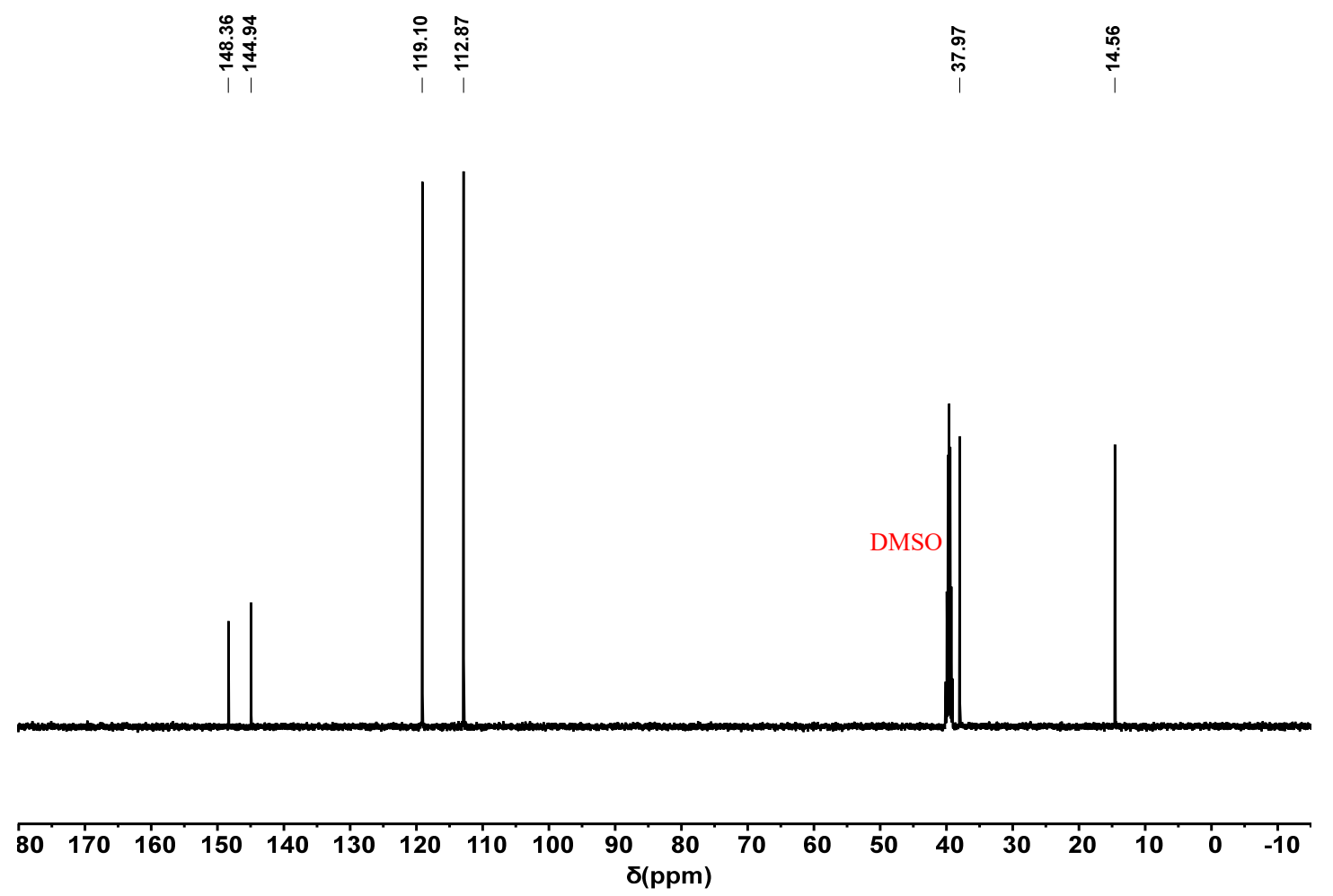

Figure S13. ${ }^{13} \mathrm{C}$ NMR $(125 \mathrm{MHz})$ spectrum of Compound 6 measured in DMSO at $25{ }^{\circ} \mathrm{C}$.

\begin{tabular}{|c|c|}
\hline : & 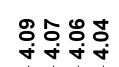 \\
\hline
\end{tabular}<smiles>CCN(O)c1ccc(Oc2ccc(N([18OH])CC)cc2)cc1</smiles>

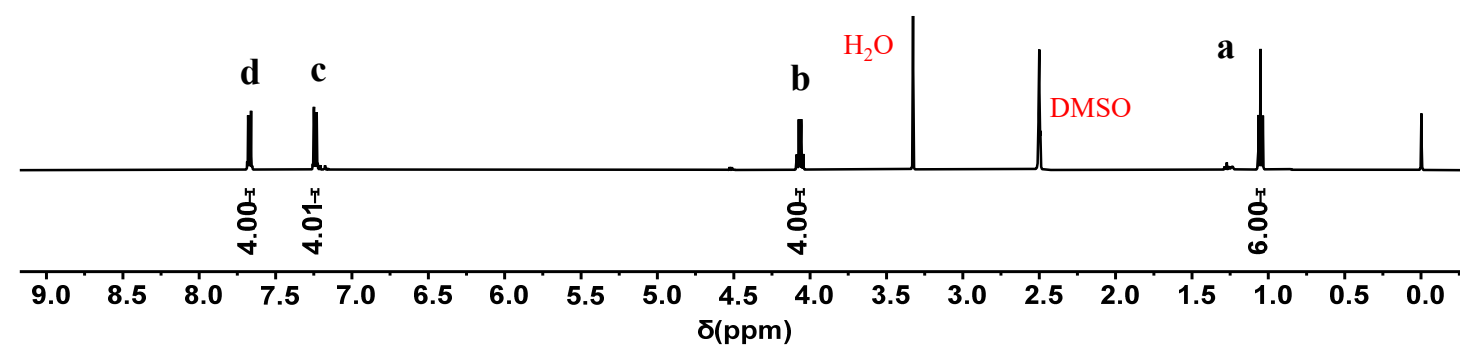

Figure S14. ${ }^{1} \mathrm{H}$ NMR $(500 \mathrm{MHz})$ spectrum of Compound 7 measured in DMSO at $25{ }^{\circ} \mathrm{C}$. 

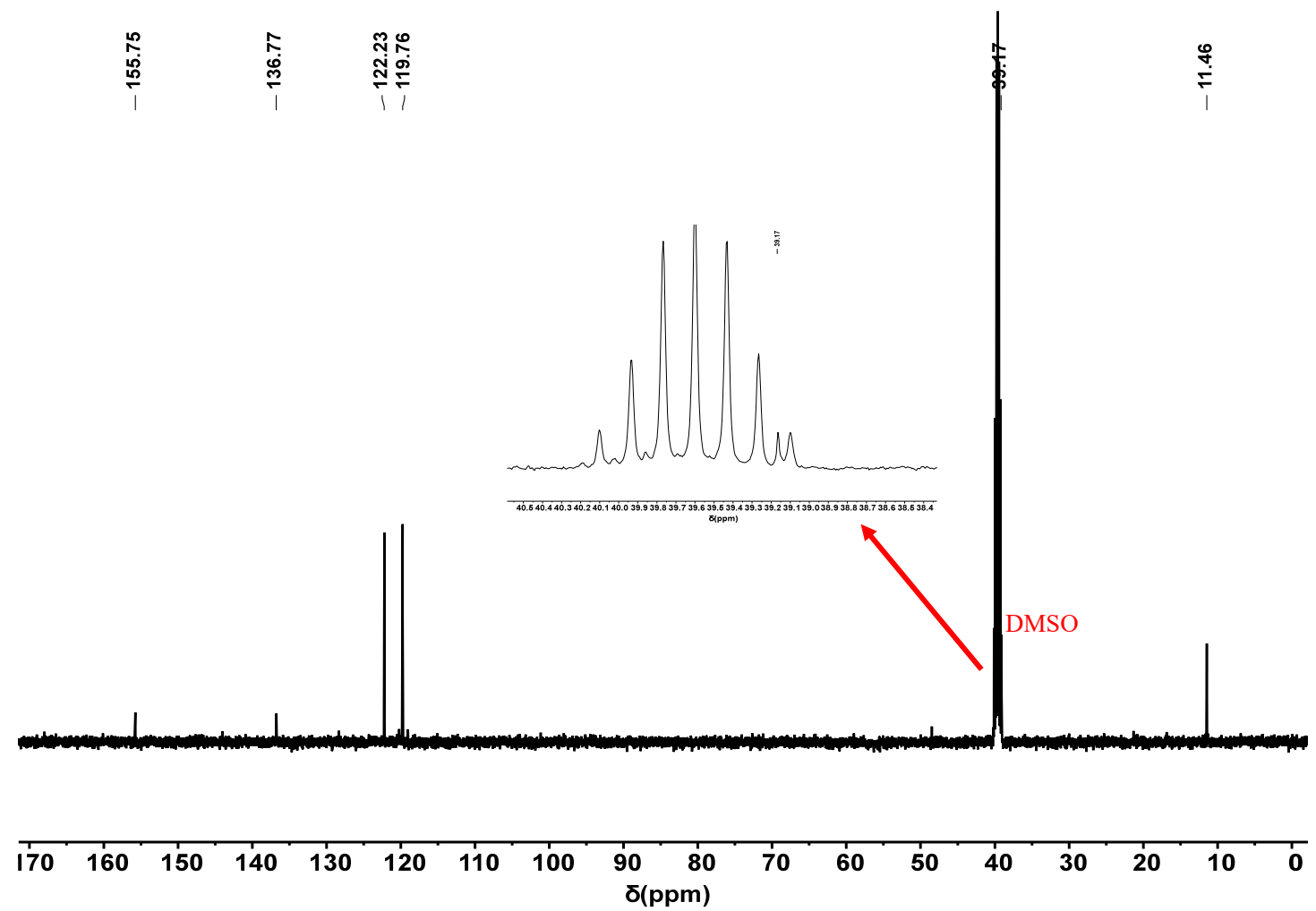

Figure S15. ${ }^{13} \mathrm{C}$ NMR $(125 \mathrm{MHz})$ spectrum of Compound 7 measured in DMSO at $25{ }^{\circ} \mathrm{C}$.

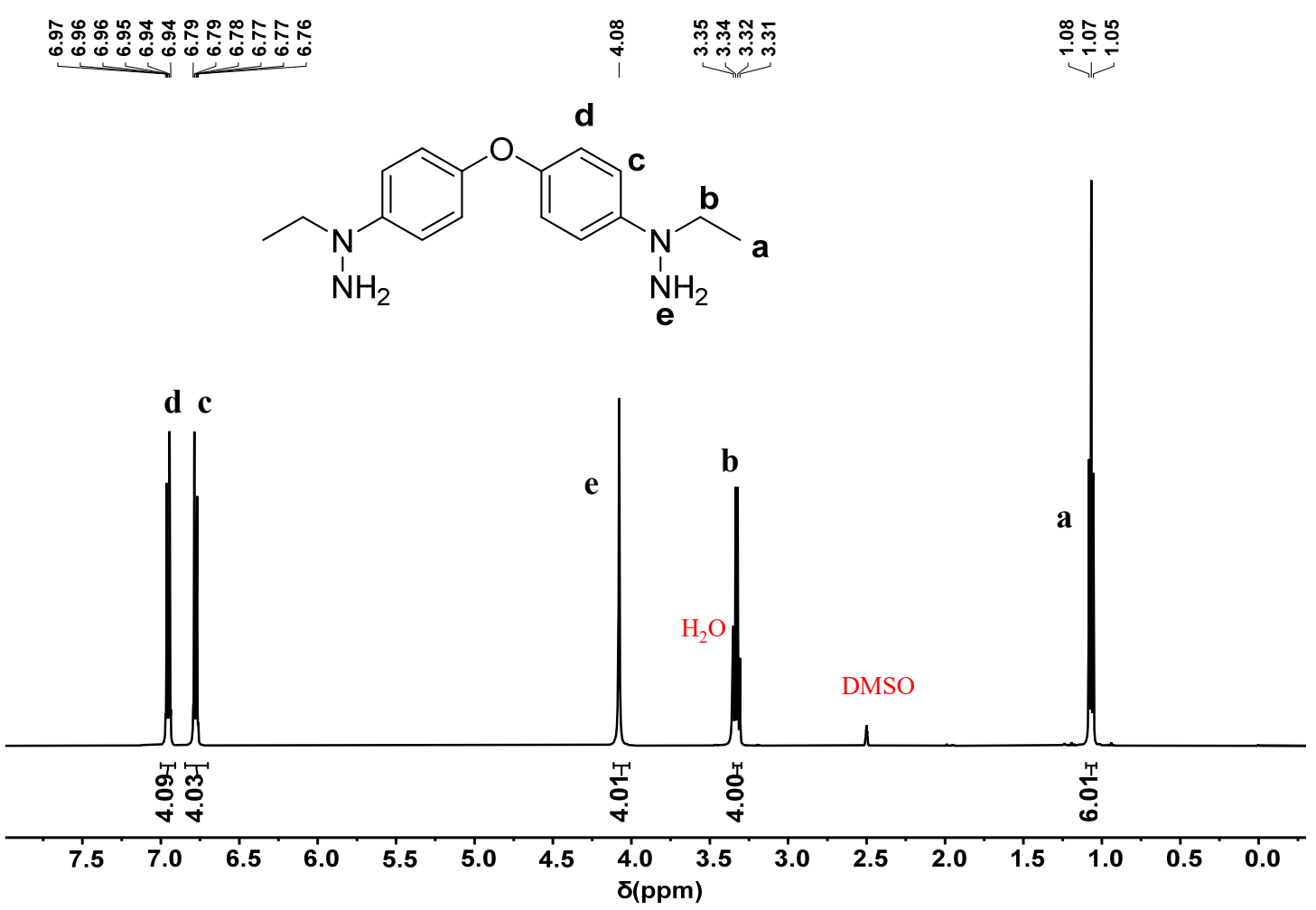

Figure S16. ${ }^{1} \mathrm{H}$ NMR $(500 \mathrm{MHz})$ spectrum of monomer II measured in DMSO at $25^{\circ} \mathrm{C}$. 

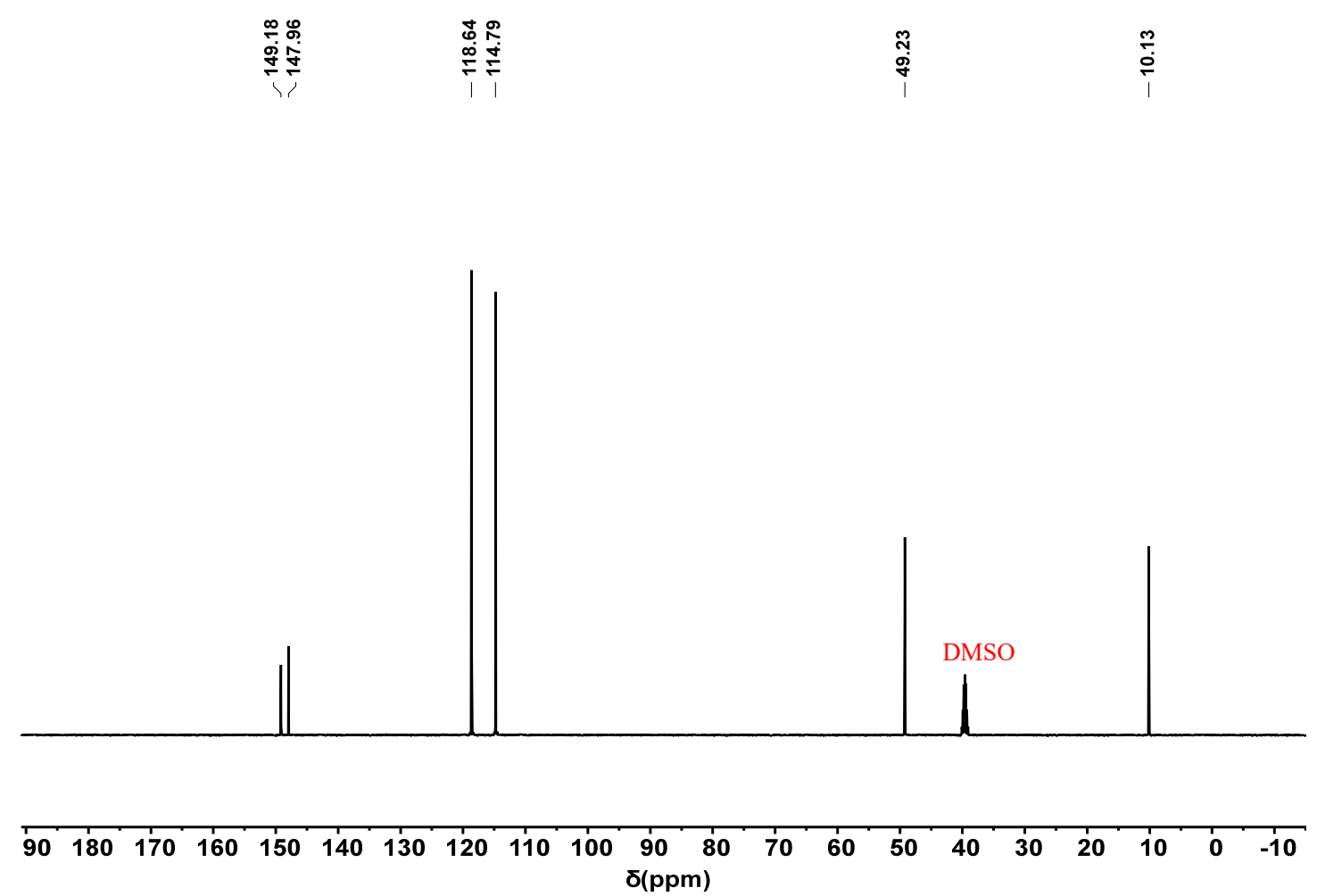

Figure S17. ${ }^{13} \mathrm{C}$ NMR $(125 \mathrm{MHz})$ spectrum of monomer II measured in DMSO at $25{ }^{\circ} \mathrm{C}$.

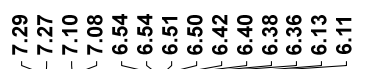<smiles>C=CC(=O)Oc1ccc(C(C)(I)c2ccc(OC(=O)C=C)cc2)cc1</smiles>

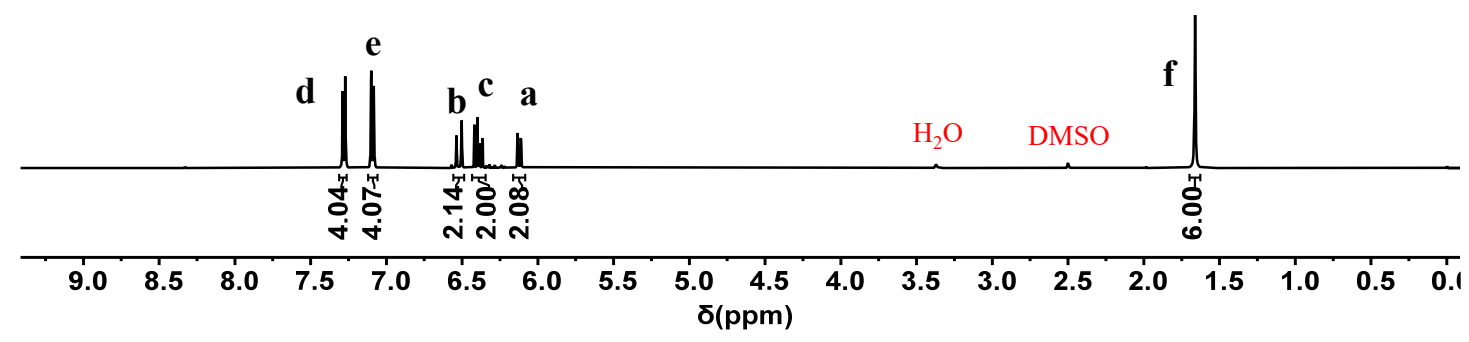

Figure S18. ${ }^{1} \mathrm{H}$ NMR $(500 \mathrm{MHz})$ spectrum of Compound $\mathbf{V}$ measured in DMSO at $25{ }^{\circ} \mathrm{C}$. 

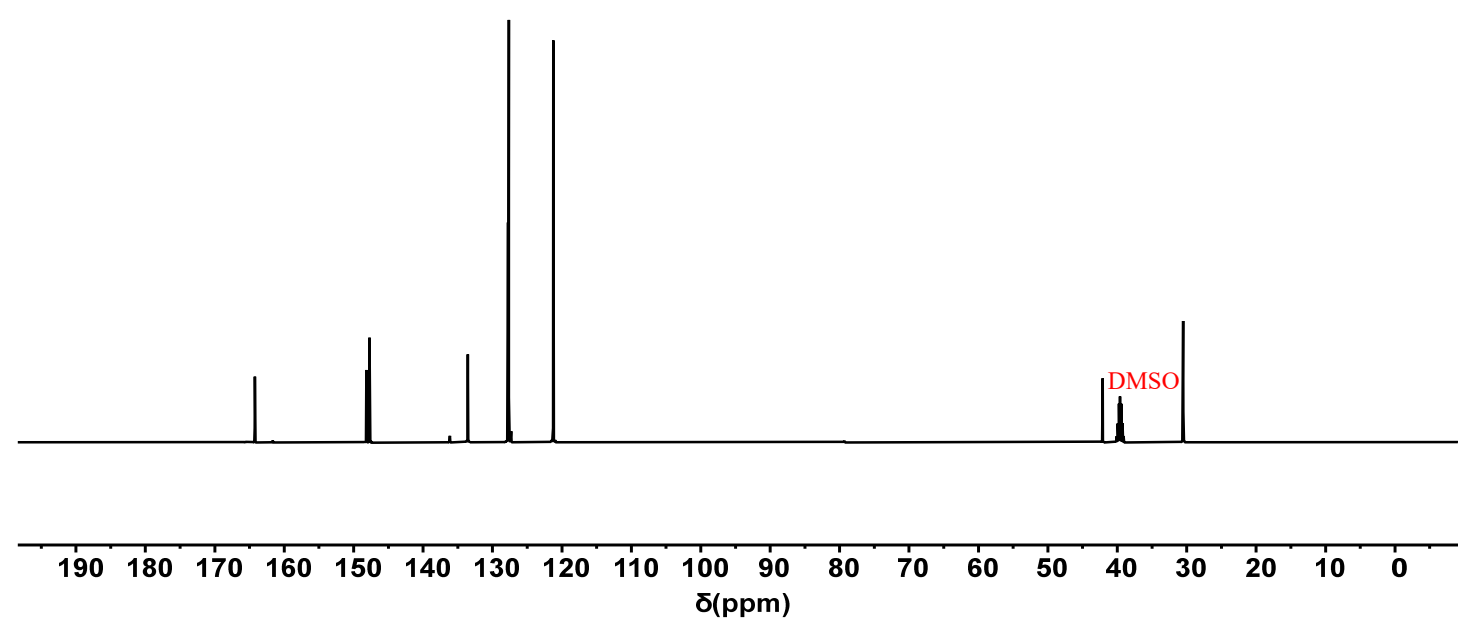

Figure S19. ${ }^{13} \mathrm{C}$ NMR $(125 \mathrm{MHz})$ spectrum of Compound $\mathbf{V}$ measured in DMSO at $25{ }^{\circ} \mathrm{C}$.
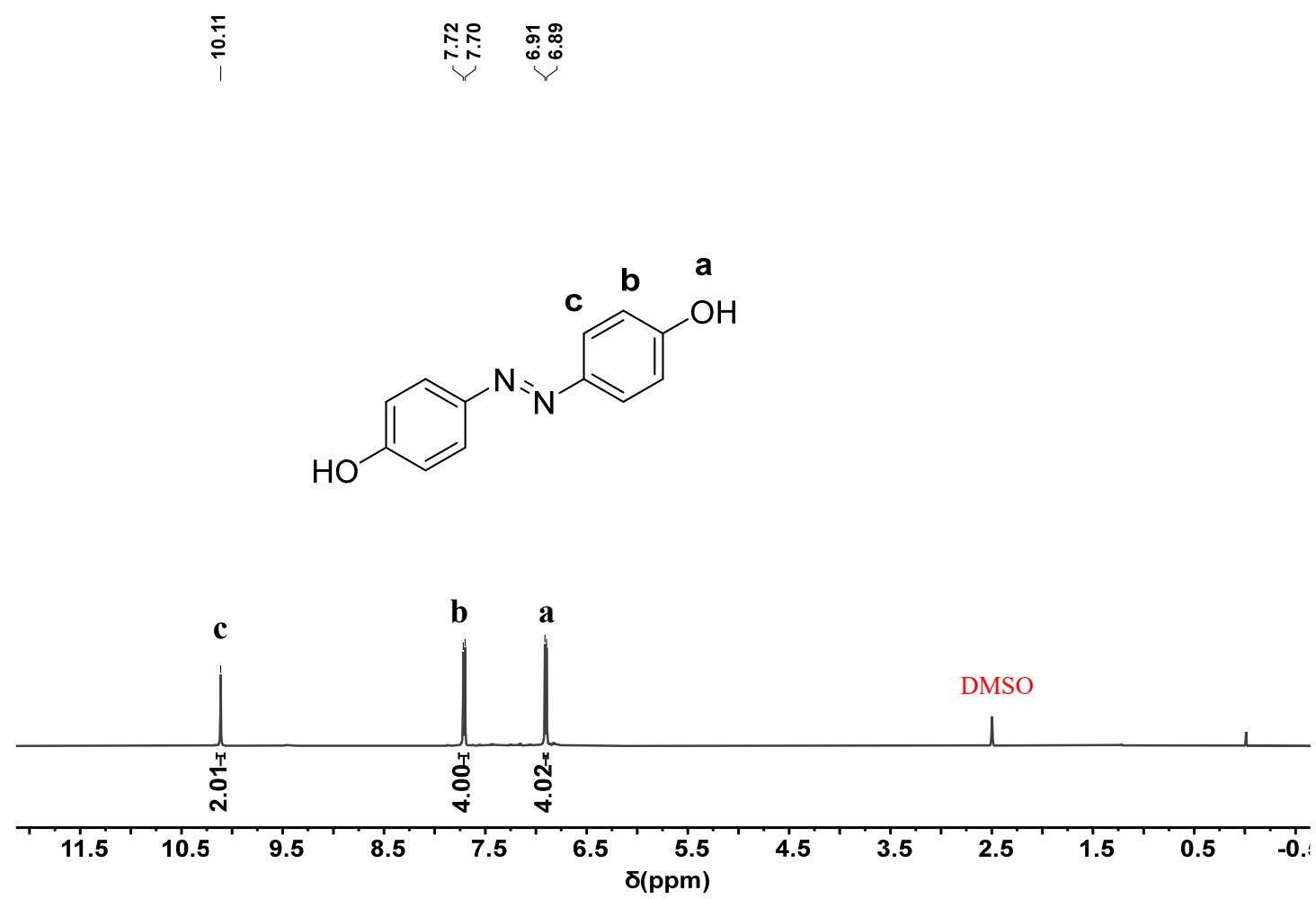

Figure S20. ${ }^{1} \mathrm{H}$ NMR $(500 \mathrm{MHz})$ spectrum of Compound Azo-OH measured in DMSO at $25{ }^{\circ} \mathrm{C}$. 


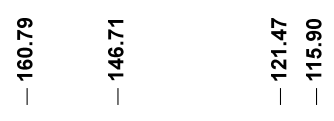

DMSO

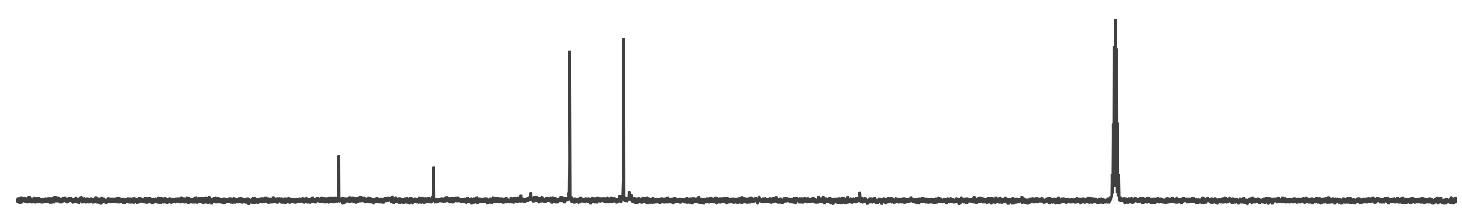

\begin{tabular}{lllllllllllllllllllllll}
\hline 10 & 200 & 190 & 180 & 170 & 160 & 150 & 140 & 130 & 120 & 110 & 100 & 90 & 80 & 70 & 60 & 50 & 40 & 30 & 20 & 10 & 0 & -10
\end{tabular} $\delta(\mathrm{ppm})$

Figure S21. ${ }^{13} \mathrm{C}$ NMR (125 MHz) spectrum of Compound Azo-OH measured in DMSO at $25{ }^{\circ} \mathrm{C}$.<smiles>C=CC(=O)Oc1ccc(N=Nc2ccc(OC(=O)C=C)cc2)cc1</smiles>

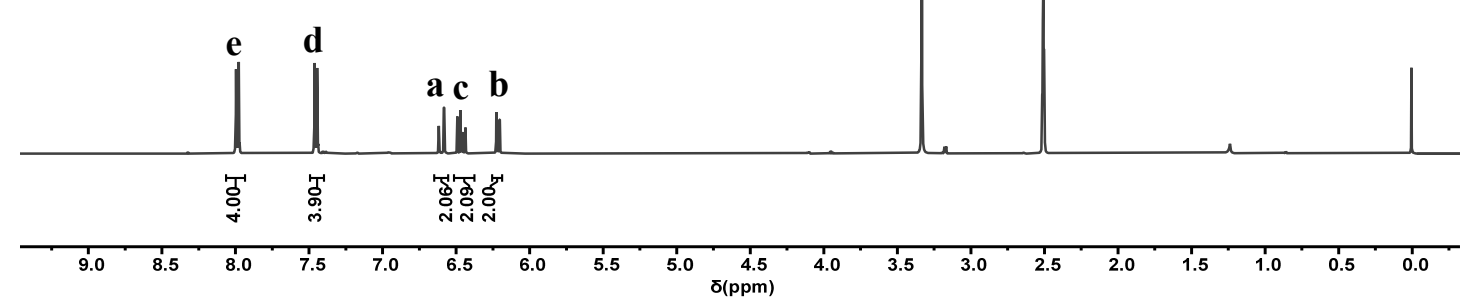

Figure S22. ${ }^{1} \mathrm{H}$ NMR $(500 \mathrm{MHz})$ spectrum of Compound VI measured in DMSO at $25^{\circ} \mathrm{C}$. 

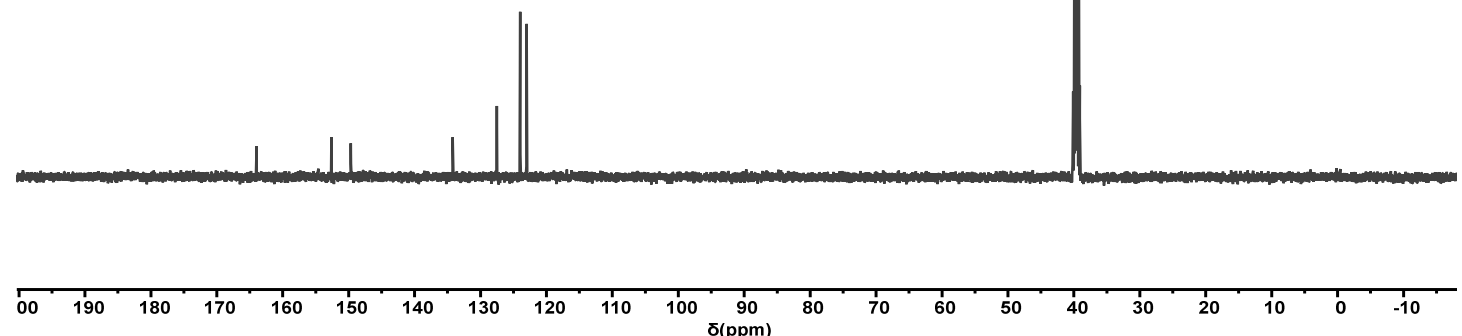

Figure S23. ${ }^{13} \mathrm{C}$ NMR $(125 \mathrm{MHz})$ spectrum of Compound VI measured in DMSO at $25{ }^{\circ} \mathrm{C}$<smiles>C=CC(=O)Oc1ccc2ccccc2c1-c1c(OC(=O)C=CI)ccc2ccccc12</smiles>

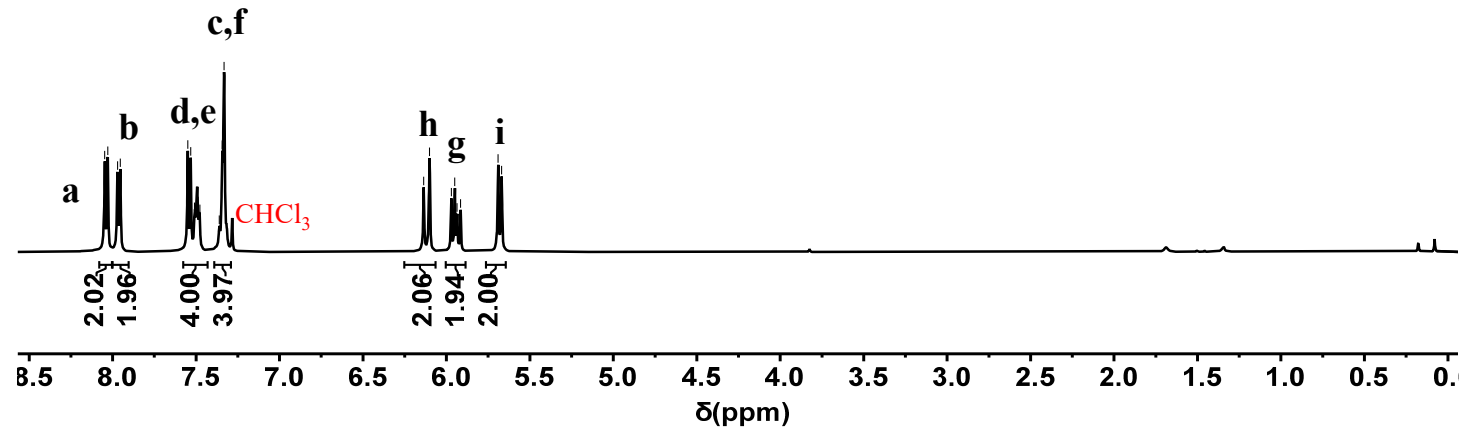

Figure S24. ${ }^{1} \mathrm{H}$ NMR $(500 \mathrm{MHz})$ spectrum of Compound $\boldsymbol{S}$-VII measured in $\mathrm{CDCl}_{3}$ at $25{ }^{\circ} \mathrm{C}$. 


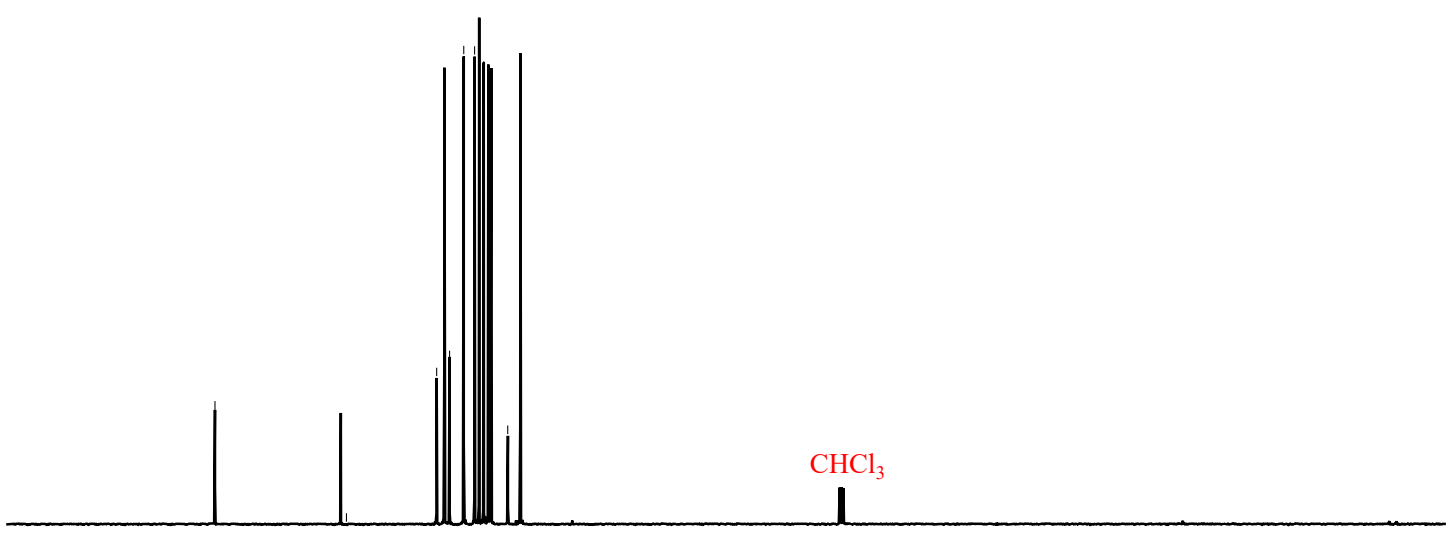

$\begin{array}{llllllllllllllllllll}190 & 180 & 170 & 160 & 150 & 140 & 130 & 120 & 110 & \begin{array}{c}100 \\ \delta(\mathrm{ppm})\end{array} & 80 & 70 & 60 & 50 & 40 & 30 & 20 & 10 & 0\end{array}$

Figure S25. ${ }^{13} \mathrm{C}$ NMR $(125 \mathrm{MHz})$ spectrum of Compound $\boldsymbol{S}$-VII measured in $\mathrm{CDCl}_{3}$ at $25{ }^{\circ} \mathrm{C}$.

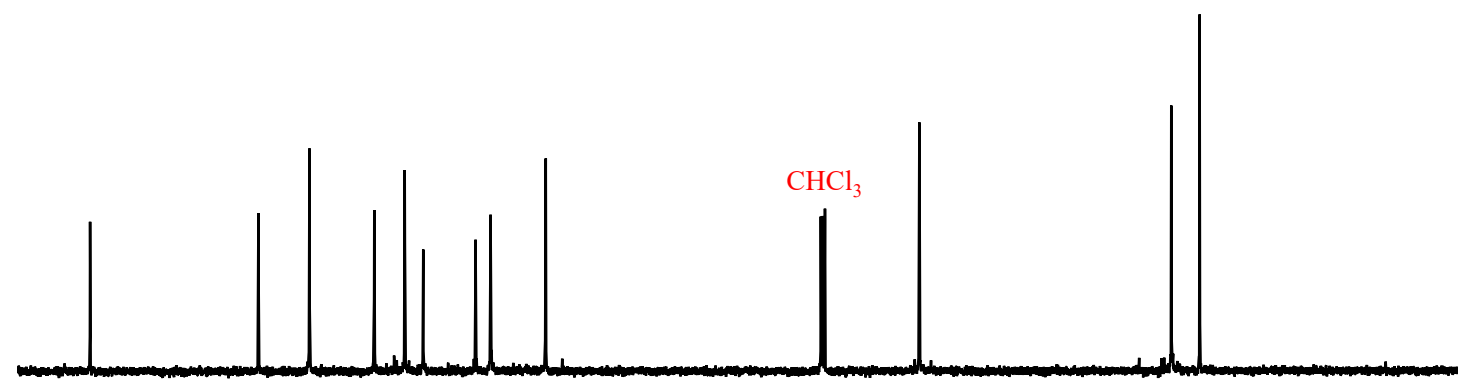

$\begin{array}{llllllllllllllllll}170 & 160 & 150 & 140 & 130 & 120 & 110 & 100 & \begin{array}{c}90 \\ \delta(\mathrm{ppm})\end{array} & 80 & 70 & 60 & 50 & 40 & 30 & 20 & 10 & C_{10}^{\prime}\end{array}$

Figure S26. ${ }^{13} \mathrm{C}$ NMR $(125 \mathrm{MHz})$ spectrum of $\mathbf{P}-\mathbf{I}$ measured in $\mathrm{CDCl}_{3}$ at $25{ }^{\circ} \mathrm{C}$. 
(a)

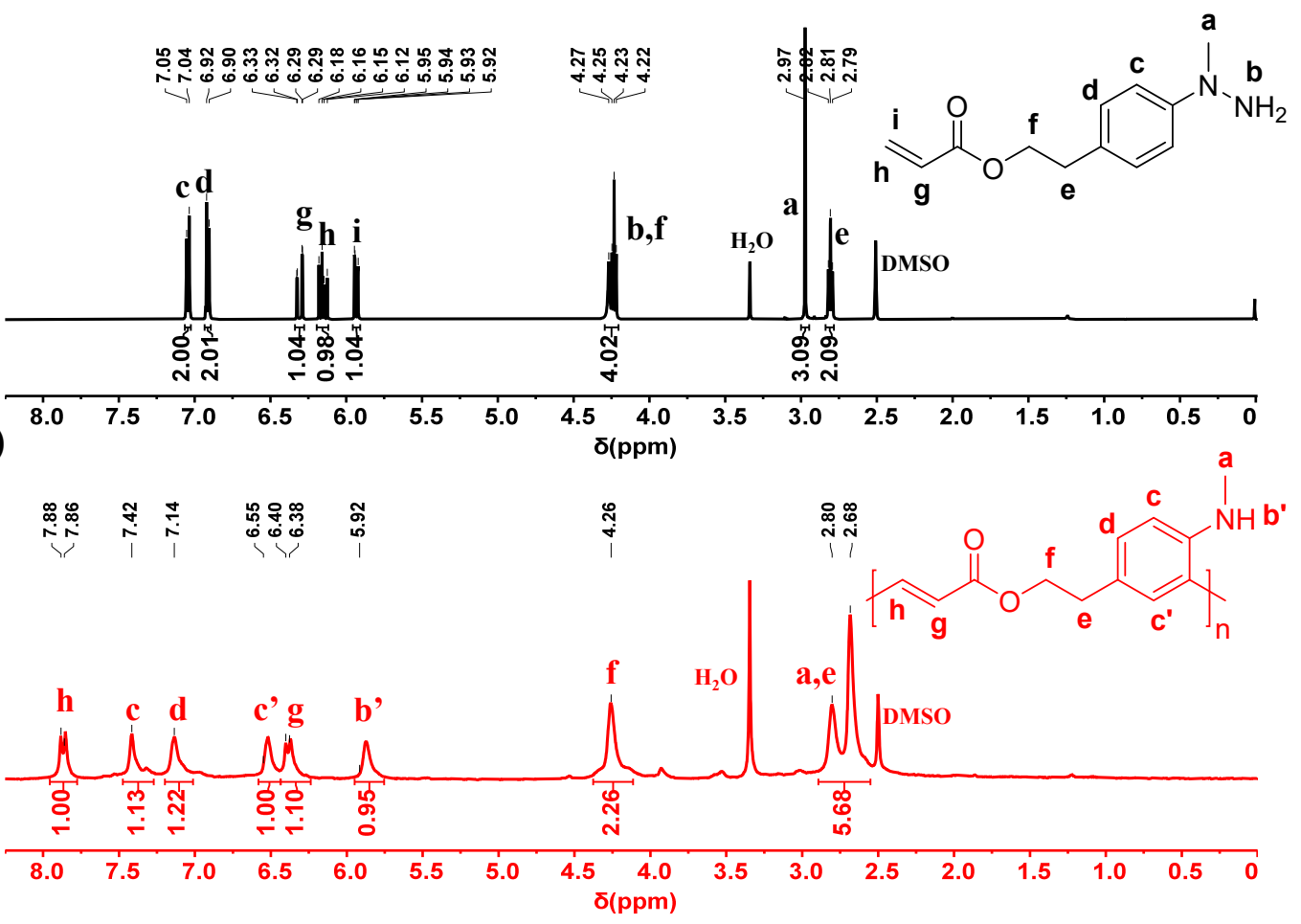

Figure S27. ${ }^{1} \mathrm{H}$ NMR (500 MHz) spectra of (a) monomer I, (b) P-I measured in DMSO at $25{ }^{\circ} \mathrm{C}$.

(a)

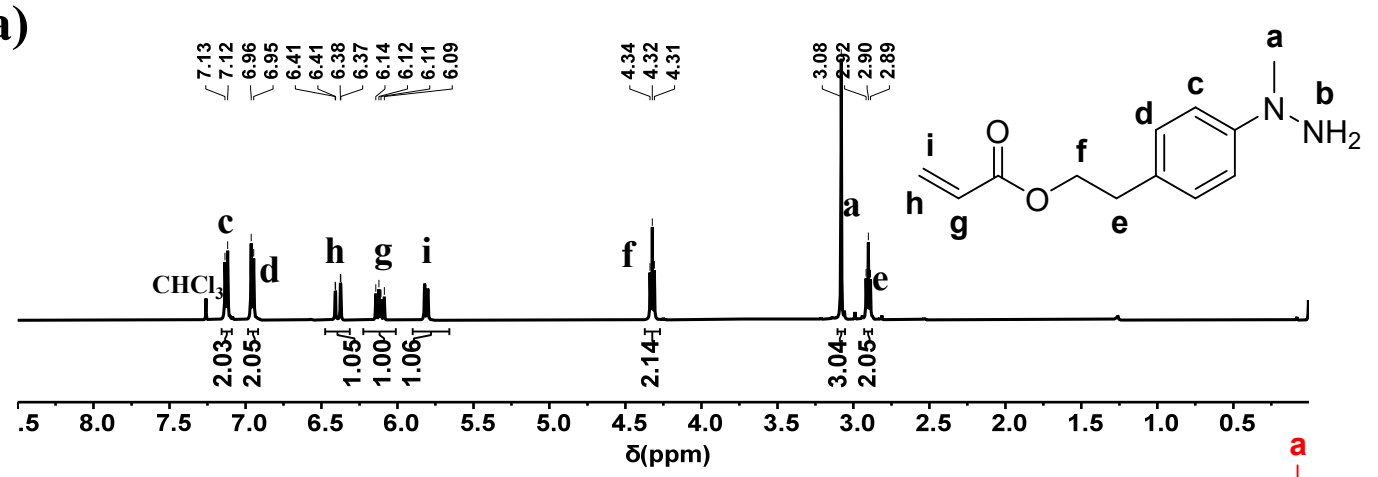

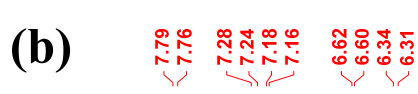

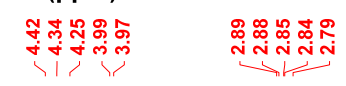

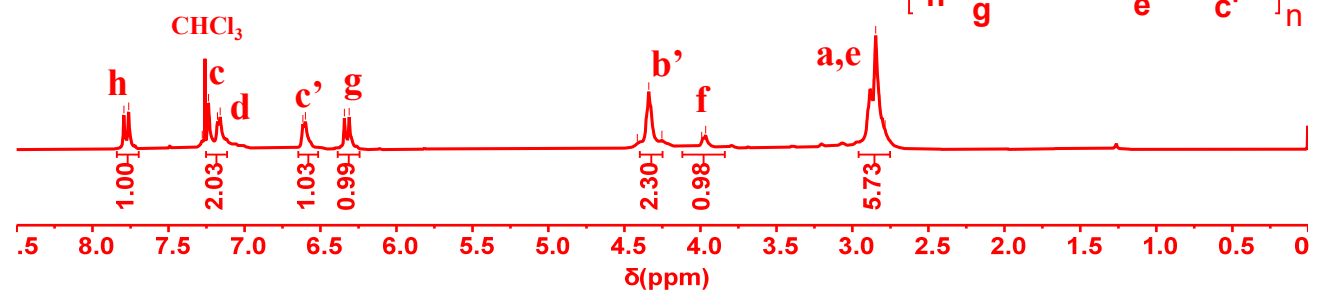

Figure S28. ${ }^{1} \mathrm{H}$ NMR (500 MHz) spectra of (a) monomer I, (b) P-I measured in $\mathrm{CDCl}_{3}$ at $25^{\circ} \mathrm{C}$. The signal at $\sim 4$ ppm for P-I is assigned to the secondary amine group, as is also typically observed after the analogous small molecule organic reactions take place. 


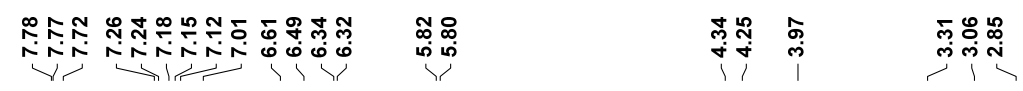

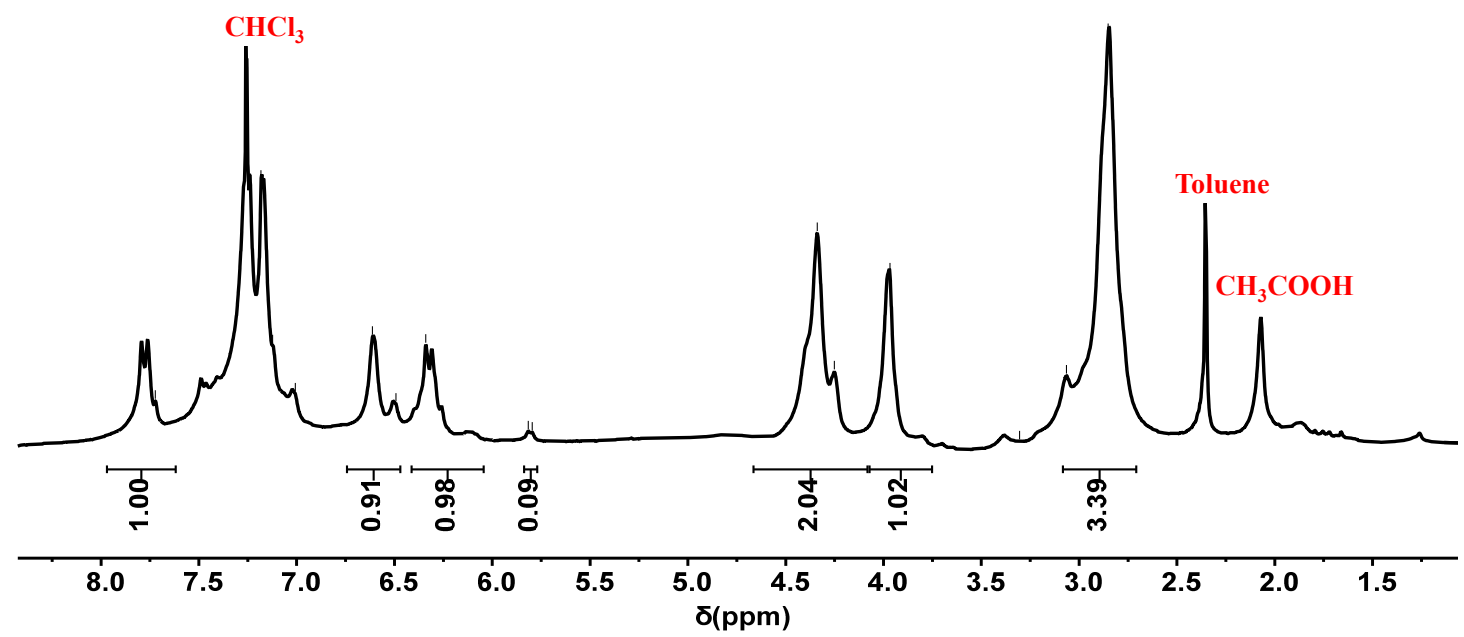

Figure S29. The ${ }^{1} \mathrm{H}$ NMR (500 MHz) spectra of monomer I with polymerization for $12 \mathrm{~h}$ in toluene.

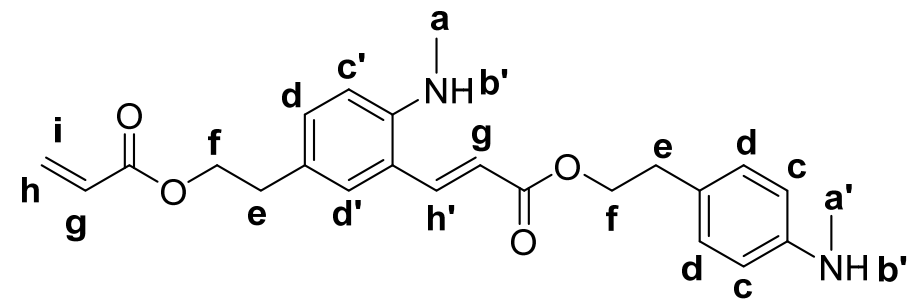

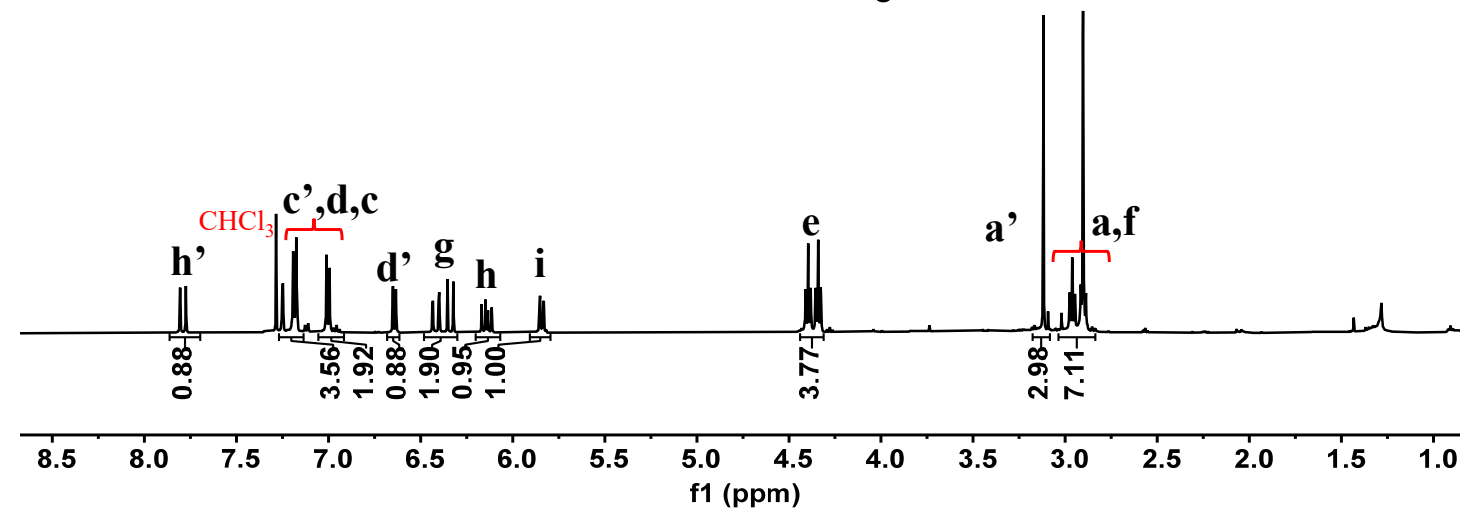

Figure S30. ${ }^{1} \mathrm{H}$ NMR $(500 \mathrm{MHz})$ spectrum of dimer measured in $\mathrm{CDCl}_{3}$ at $25{ }^{\circ} \mathrm{C}$. 


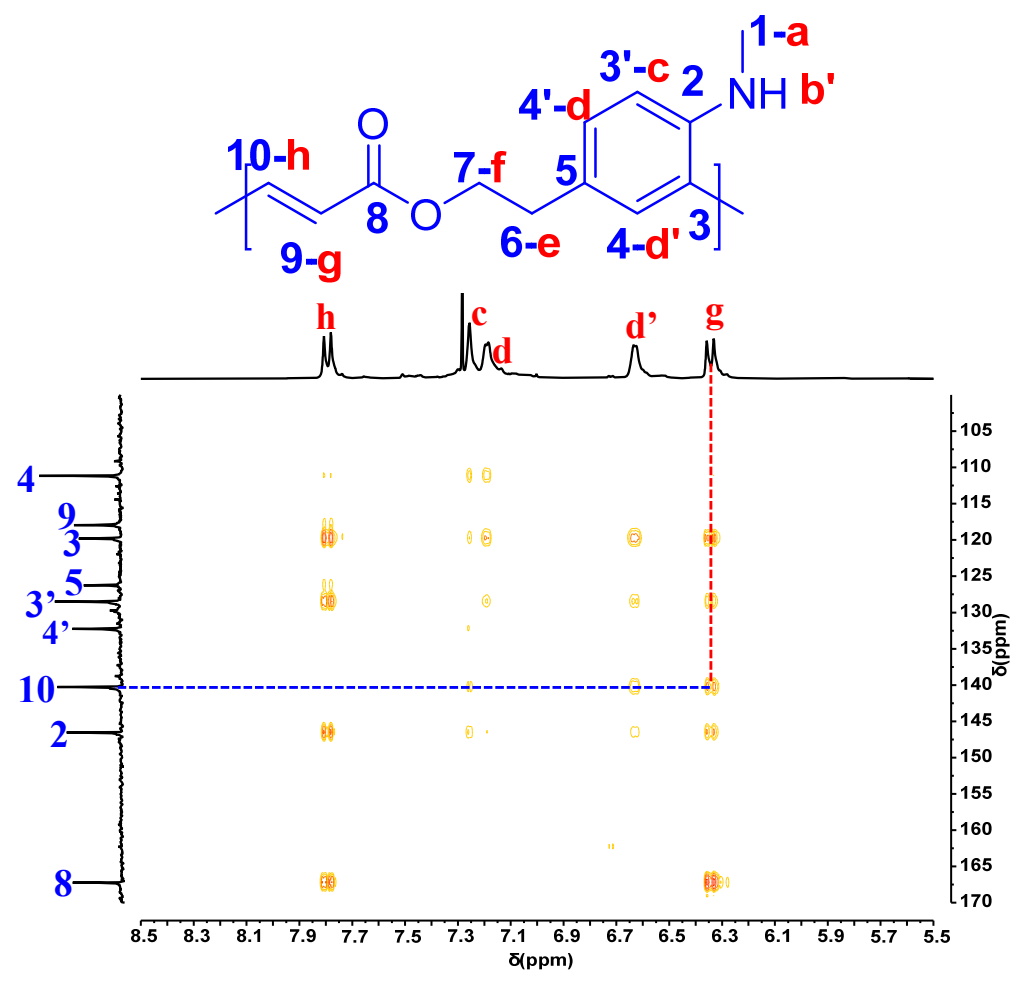

Figure S31. The HMBC (600M) spectra of P-I in $\mathrm{CDCl}_{3}$ at $25^{\circ} \mathrm{C}$.

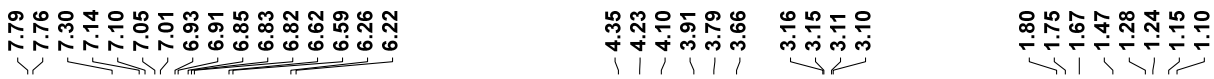

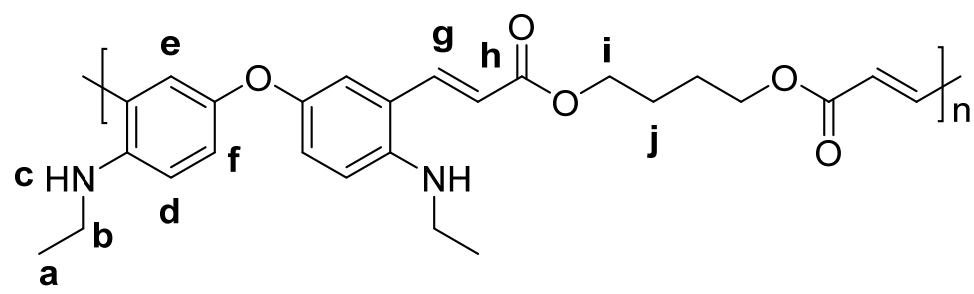
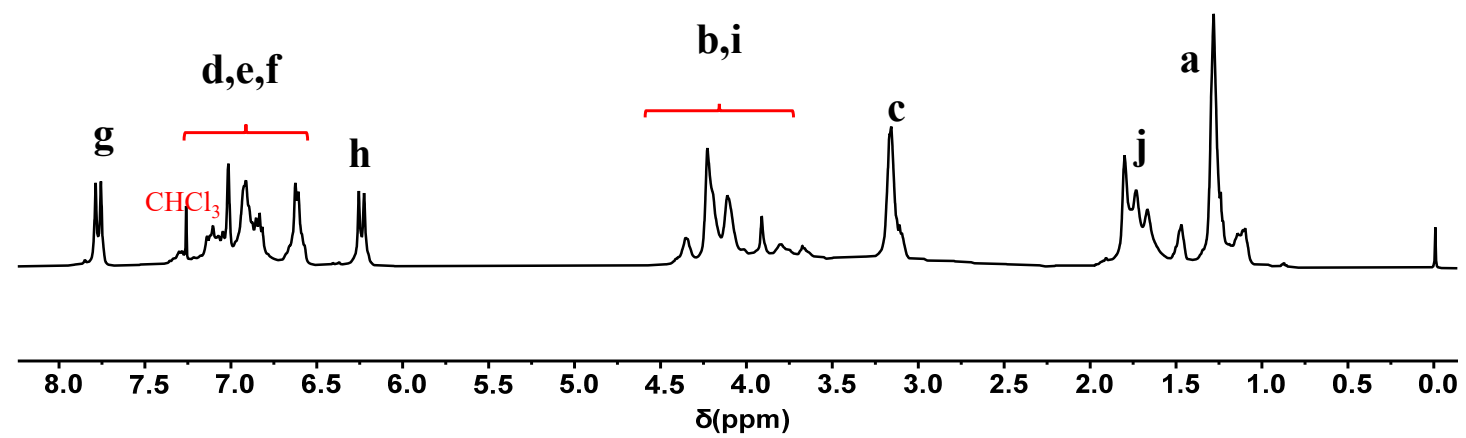

Figure S32. ${ }^{1} \mathrm{H}$ NMR $(500 \mathrm{MHz})$ spectrum of P-II,III measured in $\mathrm{CDCl}_{3}$ at $25^{\circ} \mathrm{C}$. 


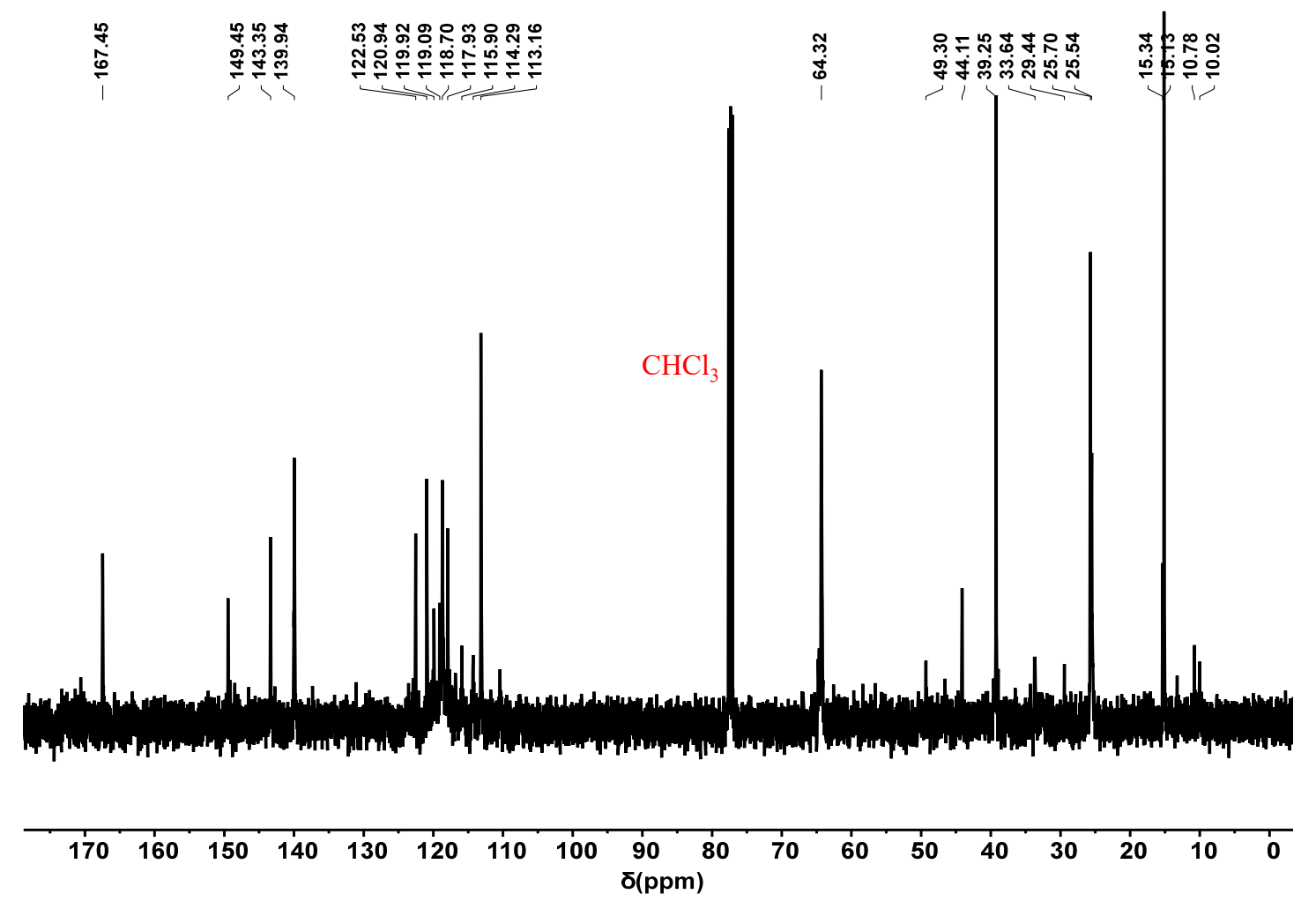

Figure S33. ${ }^{13} \mathrm{C}$ NMR $(125 \mathrm{MHz})$ spectrum of $\mathbf{P}$-II,III measured in $\mathrm{CDCl}_{3}$ at $25{ }^{\circ} \mathrm{C}$.

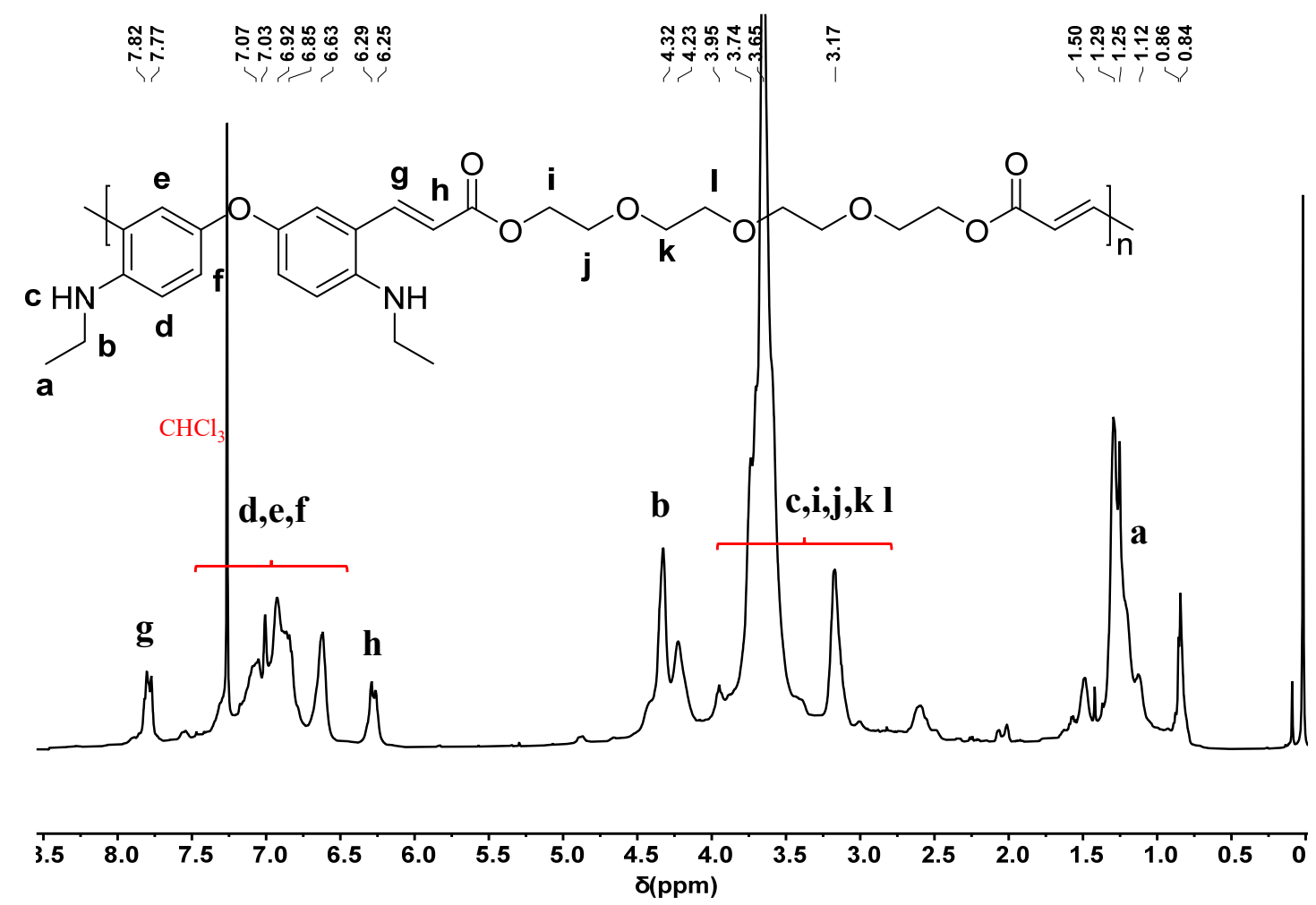

Figure S34. ${ }^{1} \mathrm{H}$ NMR $(500 \mathrm{MHz})$ spectrum of P-II,IV measured in $\mathrm{CDCl}_{3}$ at $25^{\circ} \mathrm{C}$. 

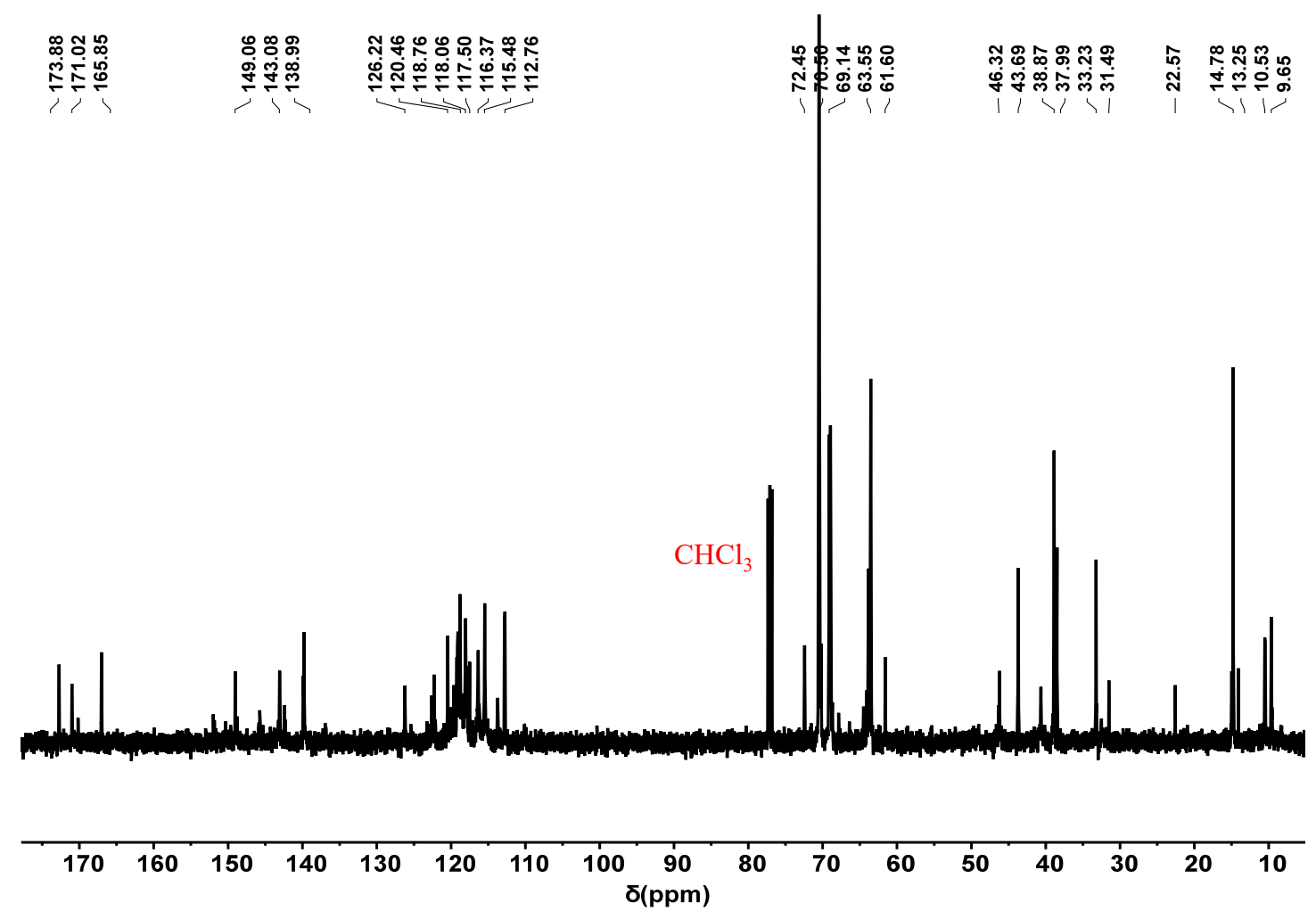

Figure S35. ${ }^{13} \mathrm{C}$ NMR $(125 \mathrm{MHz})$ spectrum of $\mathbf{P}-\mathbf{I I}, \mathbf{I V}$ measured in $\mathrm{CDCl}_{3}$ at $25{ }^{\circ} \mathrm{C}$.
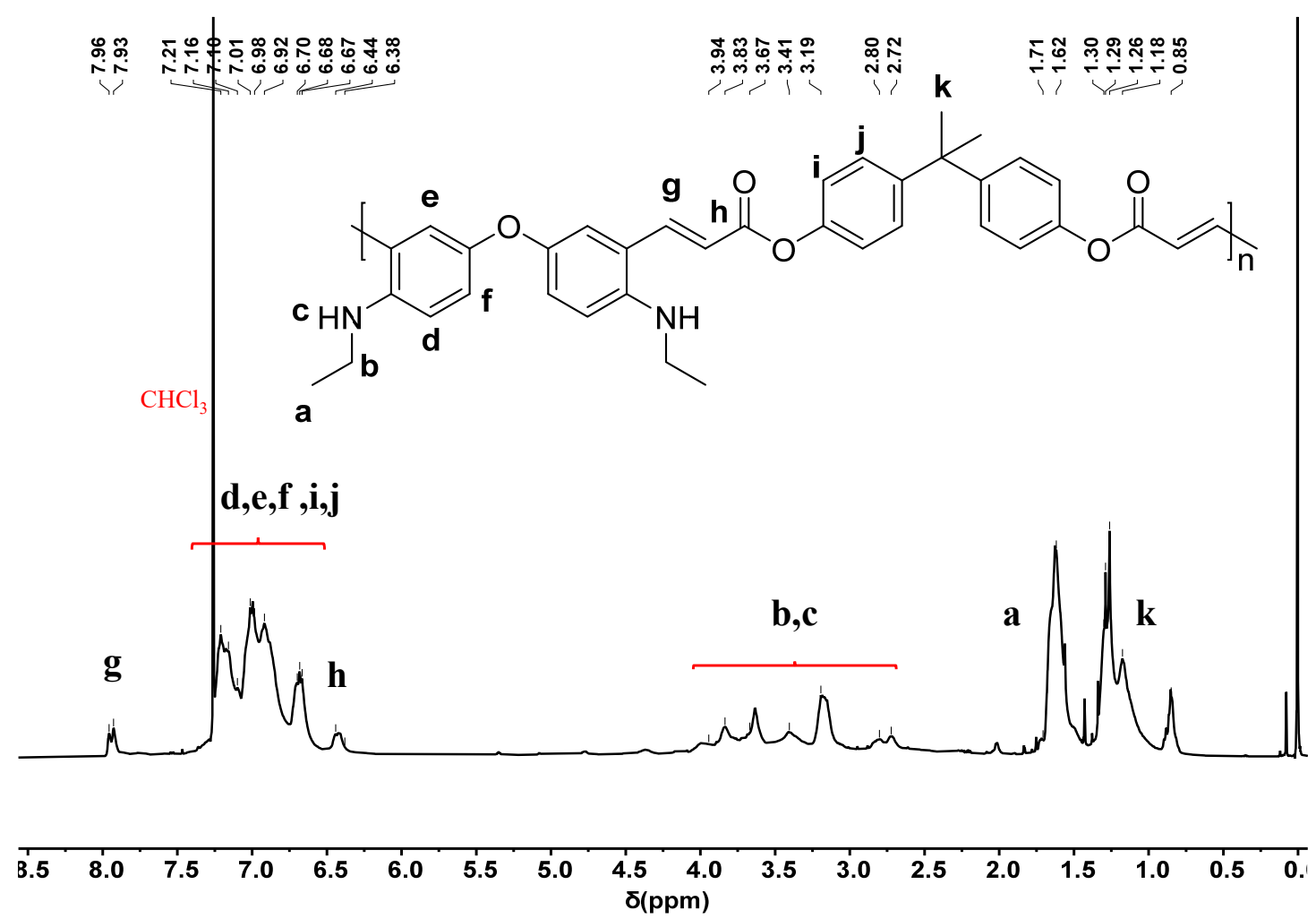

Figure S36. ${ }^{1} \mathrm{H}$ NMR (500 MHz) spectrum of $\mathbf{P}-\mathbf{I I}, \mathbf{V}$ measured in $\mathrm{CDCl}_{3}$ at $25{ }^{\circ} \mathrm{C}$. 

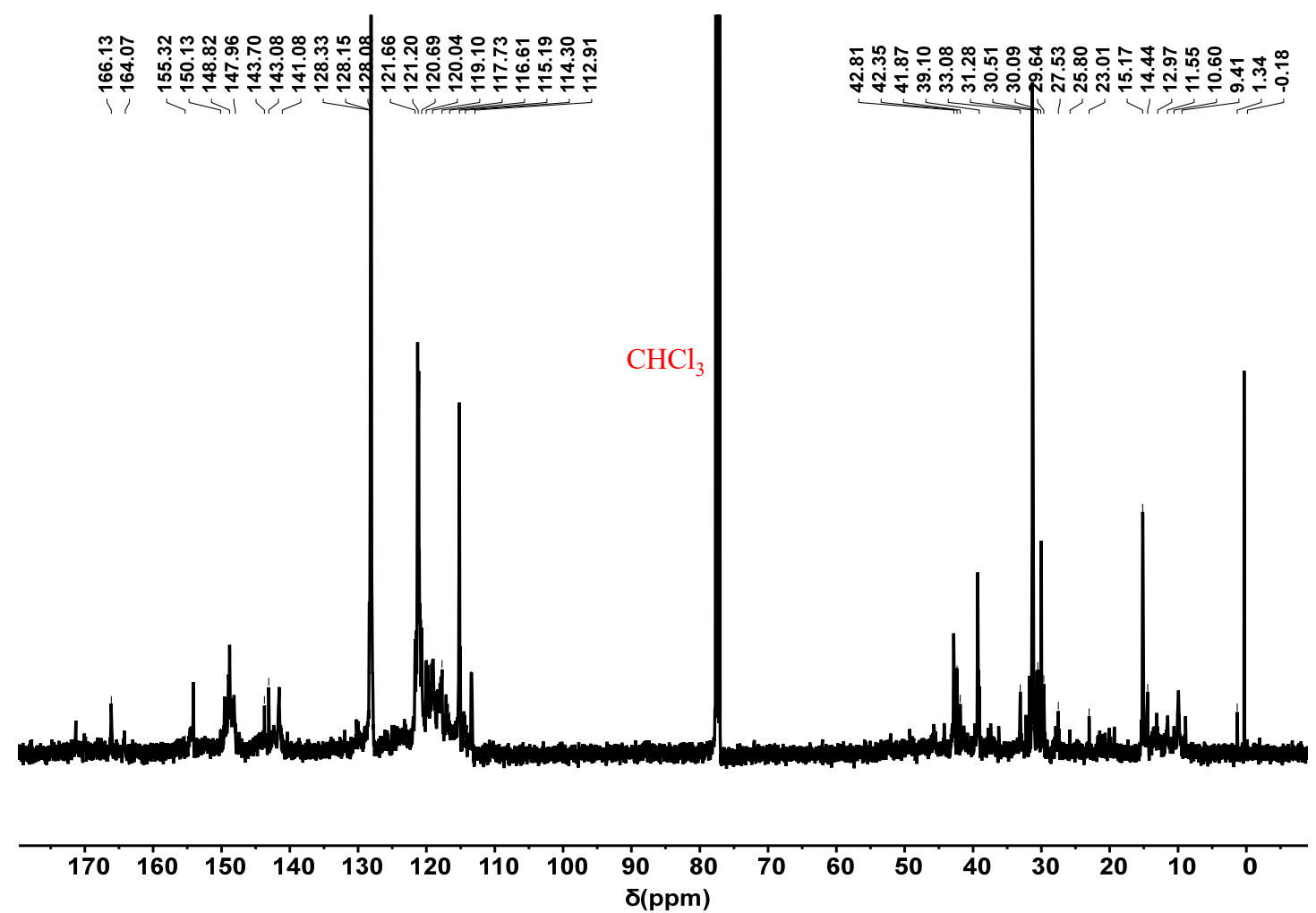

Figure S37. ${ }^{13} \mathrm{C}$ NMR $(125 \mathrm{MHz})$ spectrum of $\mathbf{P}-\mathbf{I I}, \mathbf{V}$ measured in $\mathrm{CDCl}_{3}$ at $25{ }^{\circ} \mathrm{C}$.

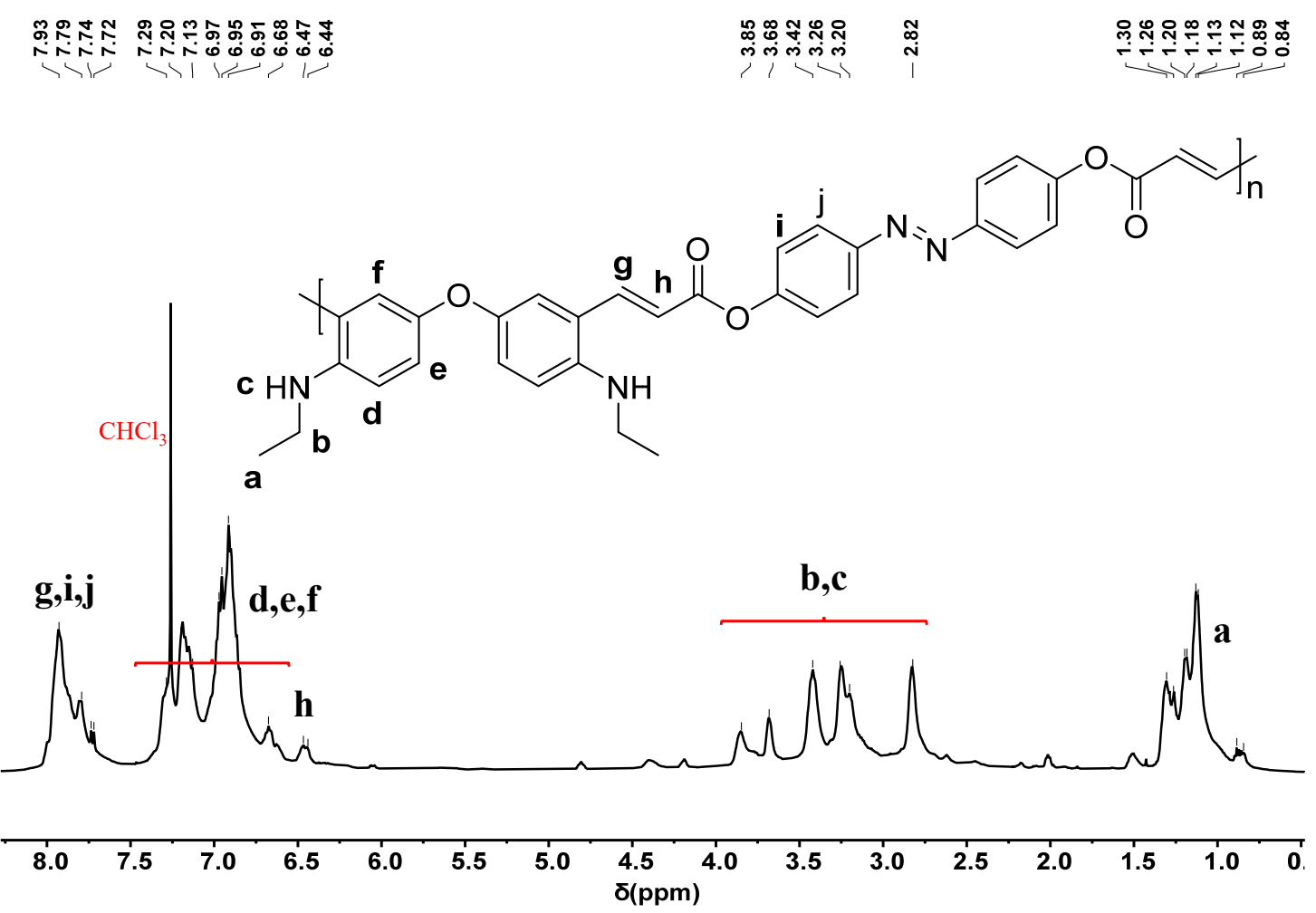

Figure S38. ${ }^{1} \mathrm{H}$ NMR $(500 \mathrm{MHz})$ spectrum of P-II,VI measured in $\mathrm{CDCl}_{3}$ at $25{ }^{\circ} \mathrm{C}$. 

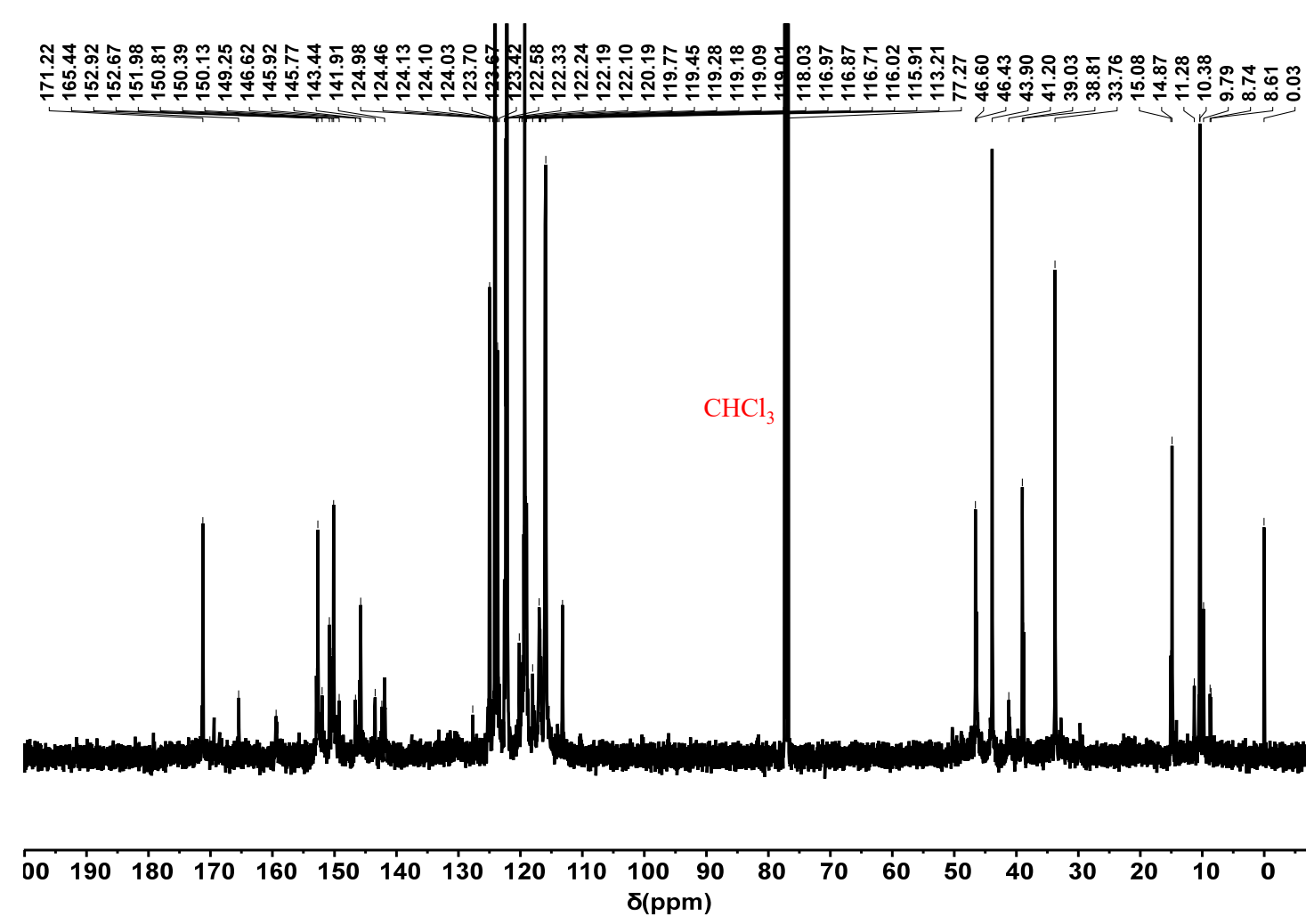

Figure S39. ${ }^{13} \mathrm{C}$ NMR (500 MHz) spectrum of P-II,VI measured in $\mathrm{CDCl}_{3}$ at $25{ }^{\circ} \mathrm{C}$.
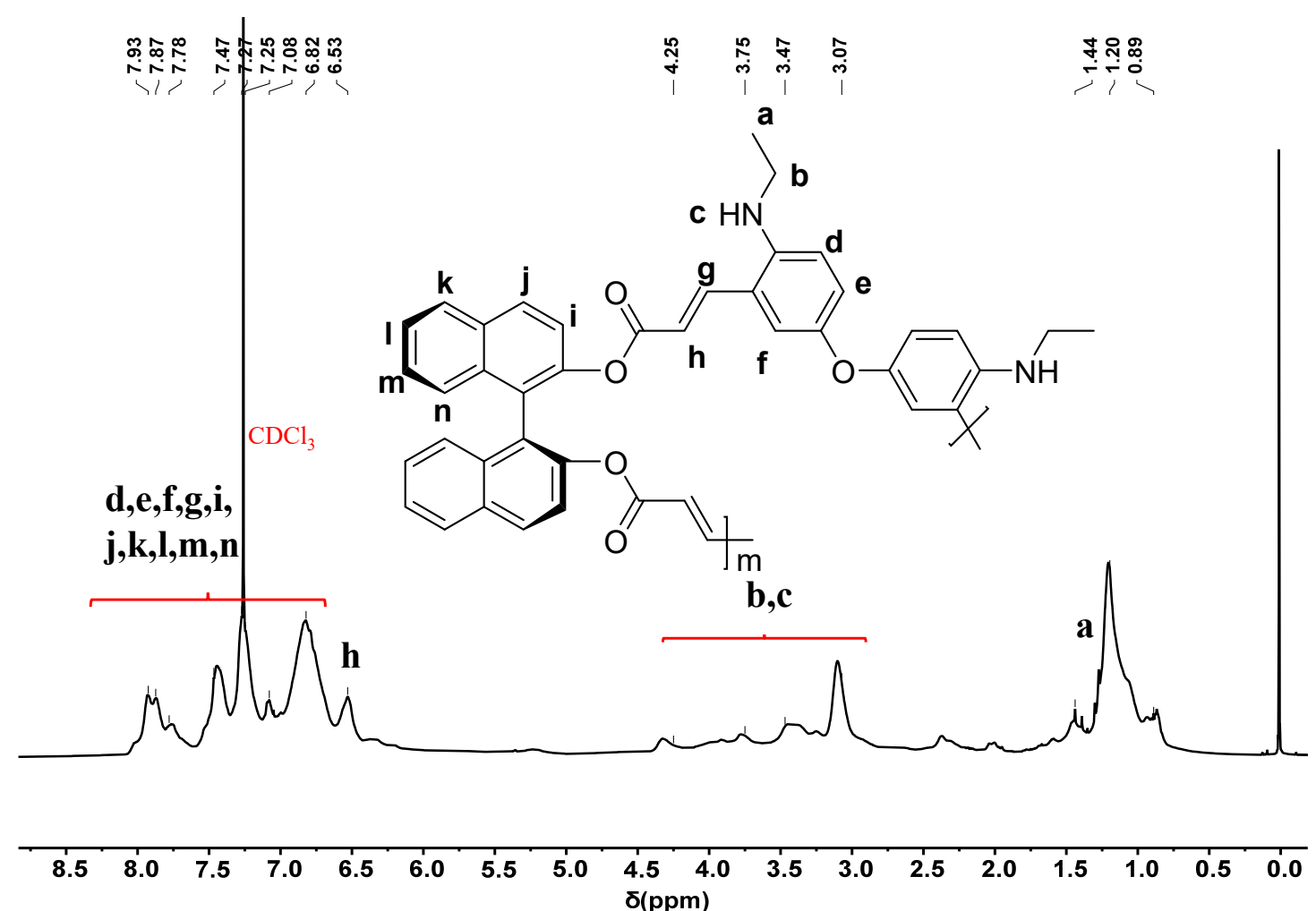

Figure S40. ${ }^{1} \mathrm{H}$ NMR $(500 \mathrm{MHz})$ spectrum of $\boldsymbol{S}$-P-II,VII measured in $\mathrm{CDCl}_{3}$ at $25{ }^{\circ} \mathrm{C}$. 


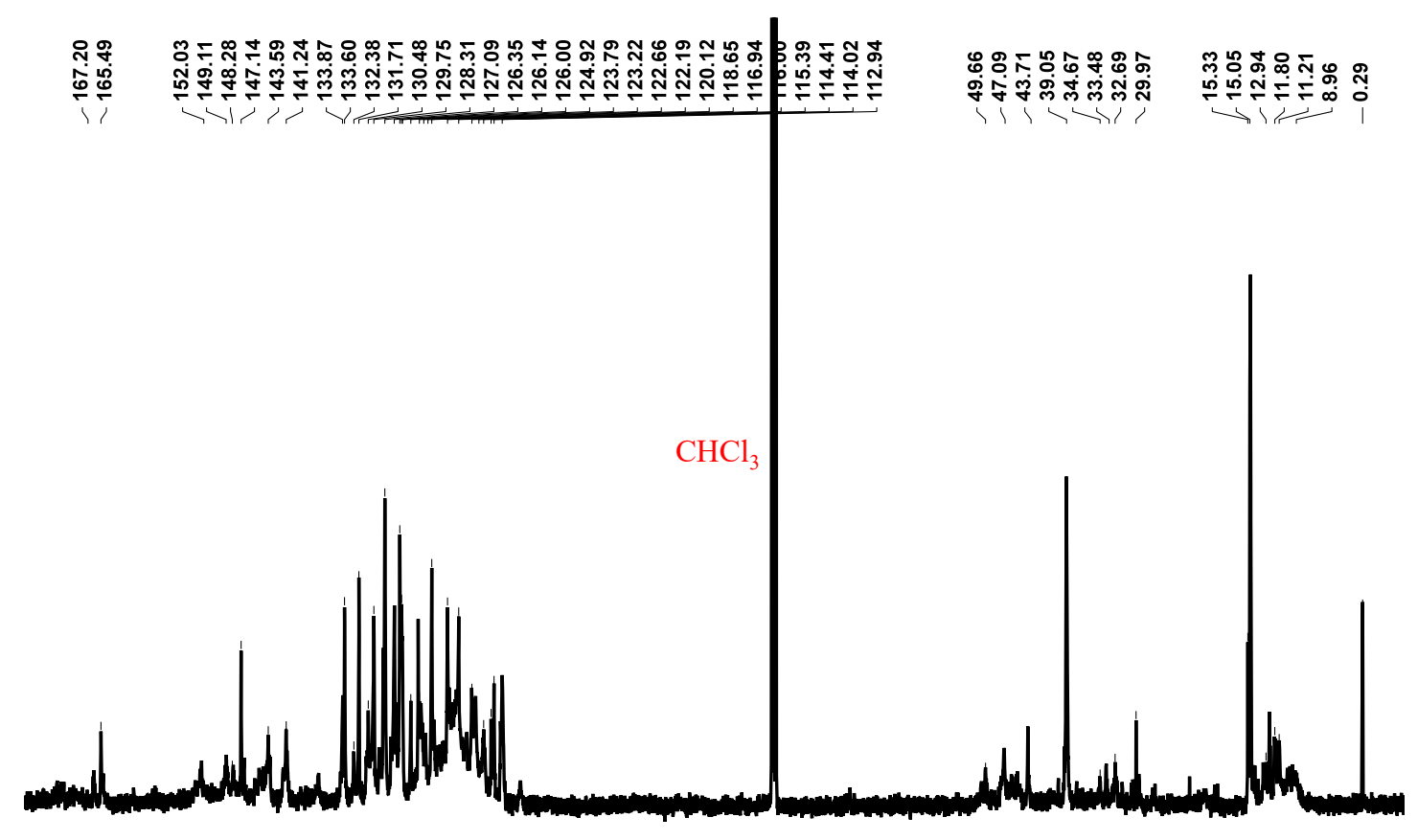

\begin{tabular}{llllllllllllllllll}
\hline 170 & 160 & 150 & 140 & 130 & 120 & 110 & 100 & $\begin{array}{c}90 \\
\delta(\mathrm{ppm})\end{array}$ & 70 & 60 & 50 & 40 & 30 & 20 & 10 & 0
\end{tabular}

Figure S41. ${ }^{13} \mathrm{C}$ NMR $(500 \mathrm{MHz})$ spectrum of $\boldsymbol{S}$-P-II, VII measured in $\mathrm{CDCl}_{3}$ at $25{ }^{\circ} \mathrm{C}$.

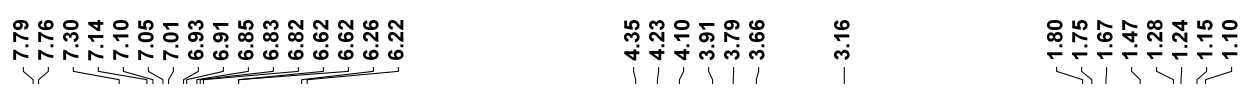<smiles>CCNc1ccc(Oc2ccc(NCC)c(C(C)(C)C)c2)cc1/C=C/C(=O)OC(C)CCCOC(=O)/C=C/C(C)(C)C</smiles>
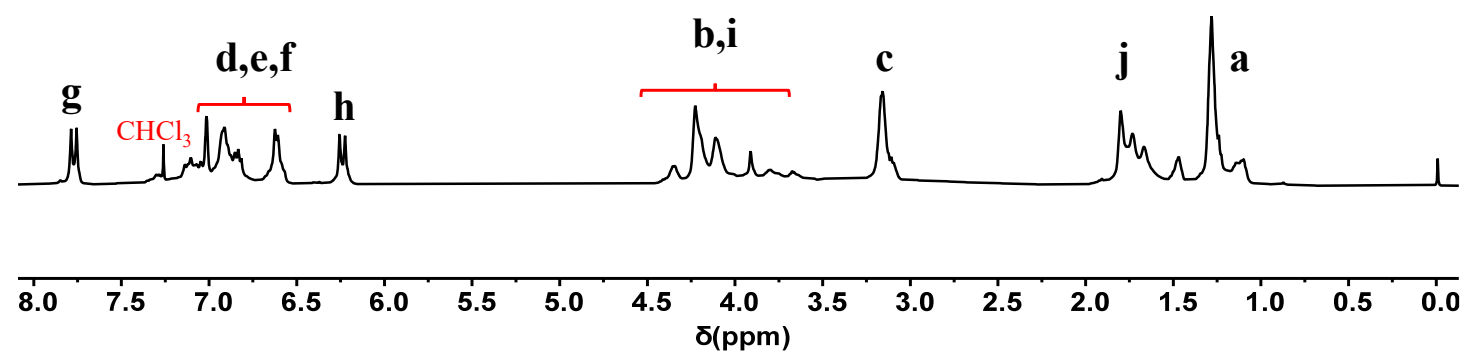

Figure S42. ${ }^{1} \mathrm{H}$ NMR $(500 \mathrm{MHz})$ spectrum of P-II,III measured in $\mathrm{CDCl}_{3}$ at $25{ }^{\circ} \mathrm{C}$ upon irradiation with $365 \mathrm{~nm}$ UV light for $30 \mathrm{~min}$. 

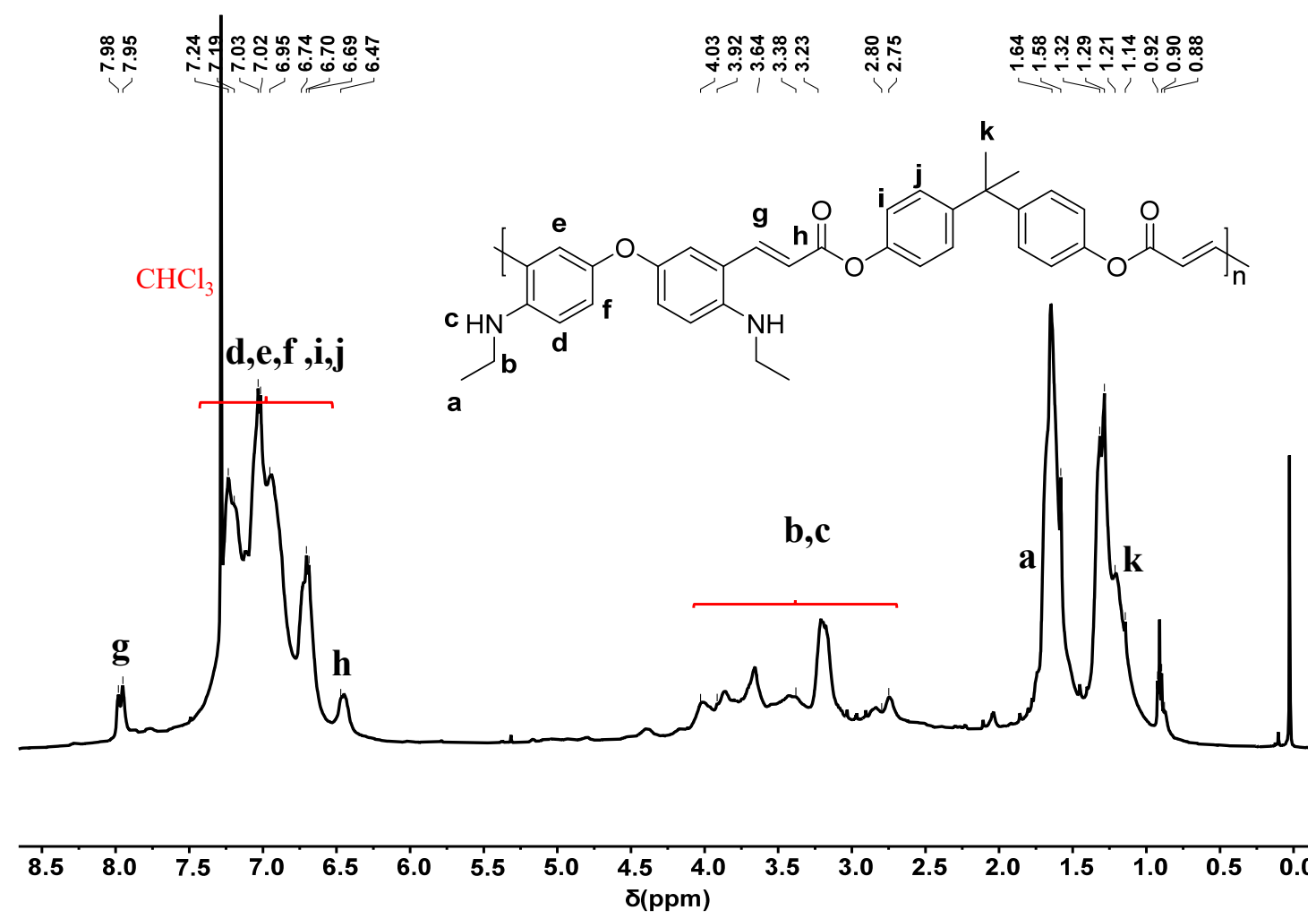

Figure S43. ${ }^{1} \mathrm{H}$ NMR $(500 \mathrm{MHz})$ spectrum of P-II,VI measured in $\mathrm{CDCl}_{3}$ at $25{ }^{\circ} \mathrm{C}$ upon irradiation with $365 \mathrm{~nm}$ UV light for $30 \mathrm{~min}$.
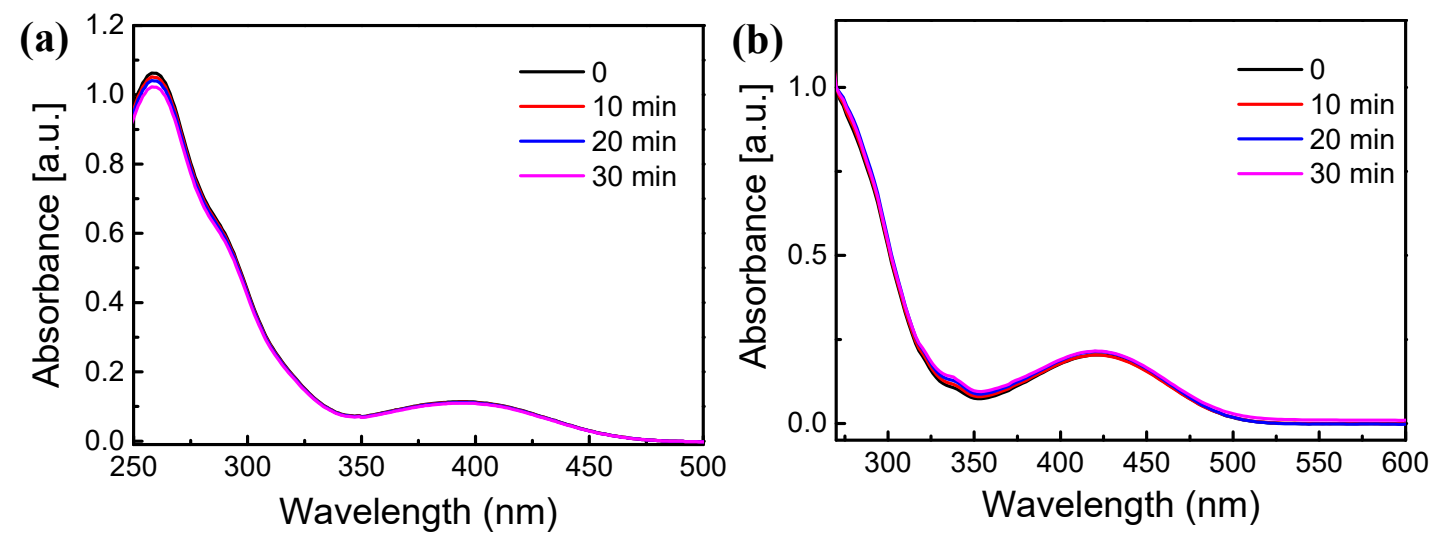

Figure S44. Time-dependent UV-vis spectral changes for a DMF solution of (a) P-II,III, and (b) P-II,V (c $=0.2 \mathrm{~g} / \mathrm{L}$ ) at $25^{\circ} \mathrm{C}$ upon irradiation with $365 \mathrm{~nm} \mathrm{UV} \mathrm{light.}$ 
(a)

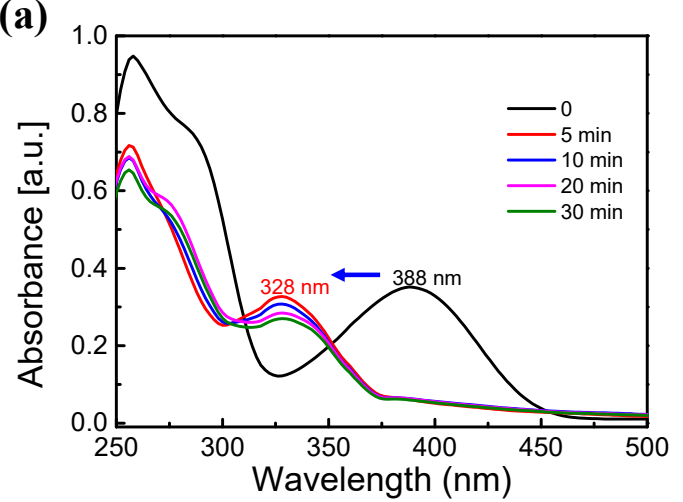

(b)

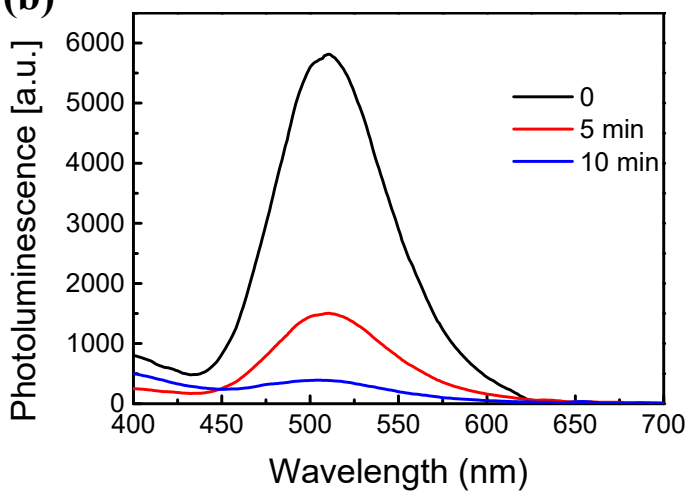

Figure S45. Time-dependent UV-vis absorption (a) and emission (b) spectral measured in $\mathrm{CHCl}_{3}$ solution of dimer $(\mathrm{c}=0.1 \mathrm{~g} / \mathrm{L}$; excitation wavelength: $260 \mathrm{~nm})$ at $25^{\circ} \mathrm{C}$ upon irradiation between $280 \mathrm{~nm}$ and $320 \mathrm{~nm}$.

(a)

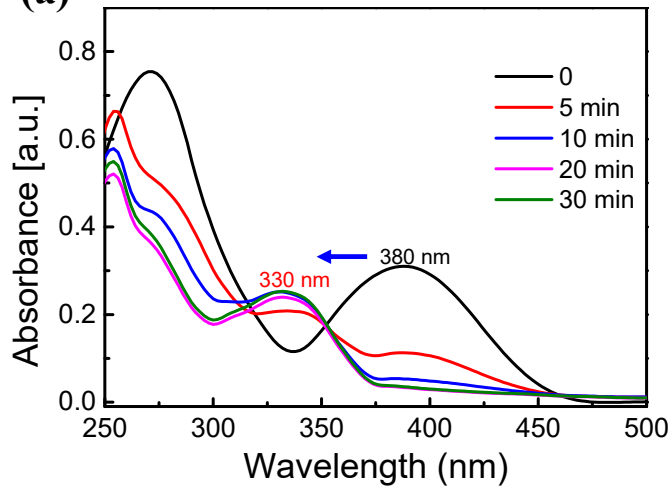

(b)

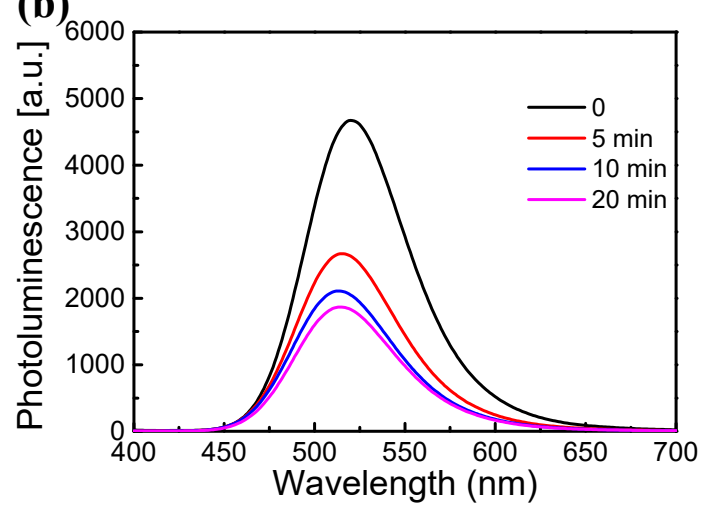

Figure S46. Time-dependent UV-vis absorption (a) and emission (b) spectral measured in $\mathrm{CHCl}_{3}$ solution of P-I $(c=0.1 \mathrm{~g} / \mathrm{L}$; excitation wavelength: $260 \mathrm{~nm})$ at $25{ }^{\circ} \mathrm{C}$ upon irradiation between $280 \mathrm{~nm}$ and $320 \mathrm{~nm}$.

(a)

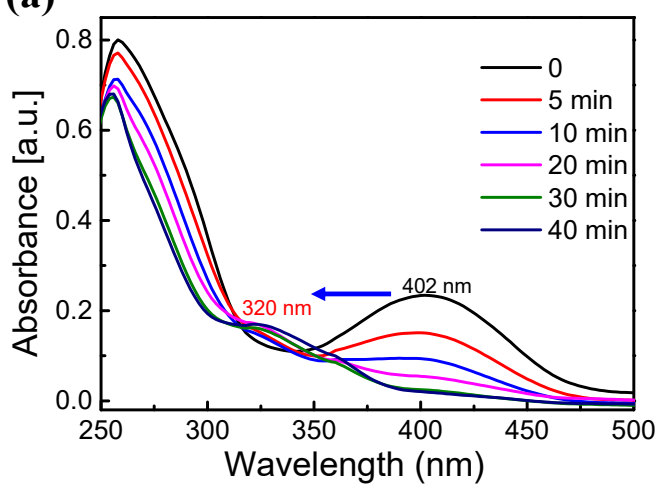

(b)

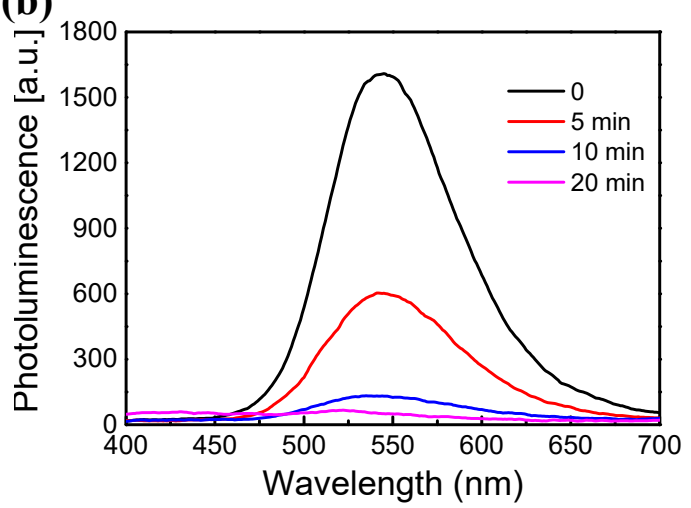

Figure S47. Time-dependent UV-vis absorption (a) and emission (b) spectral measured in $\mathrm{CHCl}_{3}$ solution of P-II,III ( $c=0.1 \mathrm{~g} / \mathrm{L}$; excitation wavelength: $260 \mathrm{~nm}$ ) at $25{ }^{\circ} \mathrm{C}$ upon irradiation between $280 \mathrm{~nm}$ and $320 \mathrm{~nm}$. 


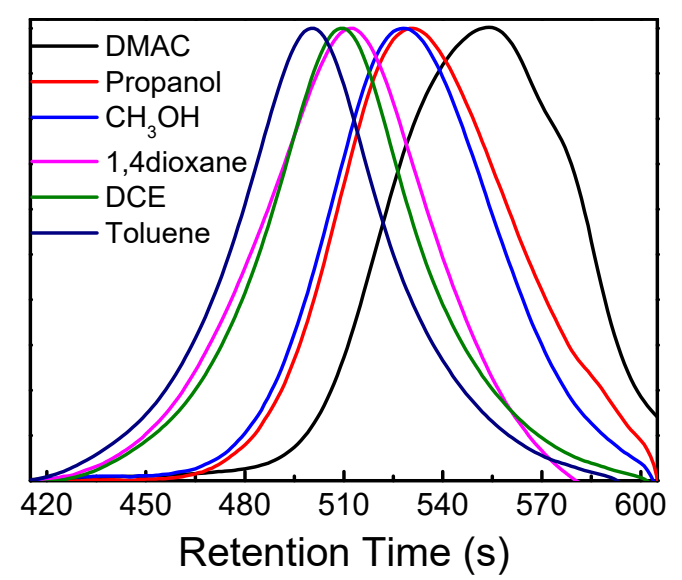

Figure S48. SEC chromatograms of the polymerization of P-I prepared by using different solvents (SEC conditions: eluent $=\mathrm{DMF}$; temperature $=35^{\circ} \mathrm{C}$.

Table S1. Polymerization of P-I by using Rh catalyst with different ratios ${ }^{a}$.

\begin{tabular}{ccccccc}
\hline Entry & $\mathrm{Rh}(\mathrm{mol} \%)$ & $\begin{array}{c}\text { Time } \\
(\mathrm{h})\end{array}$ & $\Xi^{b}$ & $M_{\mathrm{w}} / M_{\mathrm{n}}$ & $\begin{array}{c}\text { Conv. } \\
(\%)^{c}\end{array}$ & $\begin{array}{c}\text { Yield } \\
(\%)^{d}\end{array}$ \\
1 & 0.1 & 24 & 5.6 & 1.31 & 67.1 & 56.5 \\
2 & 0.5 & 12 & 8.3 & 1.66 & 69.3 & 59.1 \\
3 & 1 & 12 & 10.1 & 1.31 & 86.4 & 72.0 \\
4 & 2 & 12 & 12.4 & 1.23 & 92.7 & 86.2 \\
5 & 4 & 12 & 6.5 & 1.25 & 74.4 & 62.0 \\
$6^{\text {e }}$ & 2 & 12 & 9.9 & 1.34 & 81.0 & 63.0
\end{tabular}

${ }^{a}$ Polymerization conditions: Toluene was used as solvent and HOAc was 1.2 eq. ${ }^{b}$ Determind by DMF size-exclusion chromatography (SEC) calibrated by using polystyrene (PS) standards. ${ }^{c}$ Conversion after precipitated from diethyl ether. ${ }^{d}$ Isolated yield after purification by $\mathrm{Et}_{2} \mathrm{O}$ from THF. ${ }^{e}$ The $\left[\mathrm{RhCp}^{*} \mathrm{Cl}_{2}\right]_{2} / 4 \mathrm{AgSbF}_{6}$ is used catalyst.

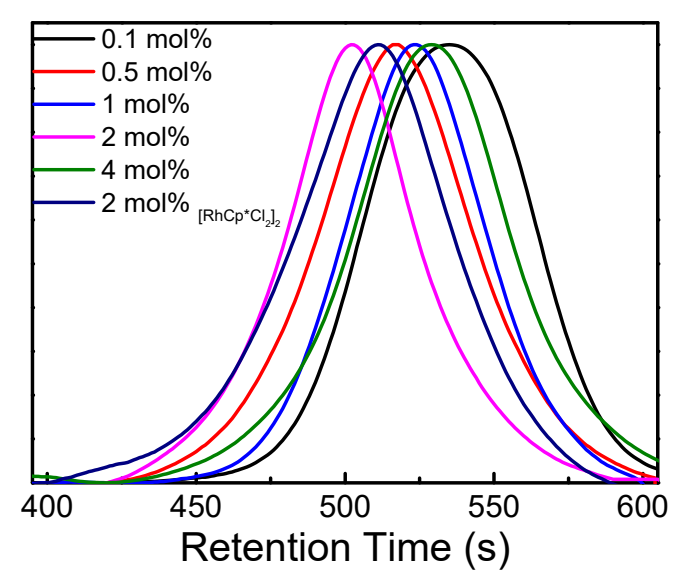

Figure S49. SEC chromatograms of the polymerization of P-I prepared by using Rh catalyst with different ratio $\left(\mathrm{SEC}\right.$ conditions : eluent $=\mathrm{DMF}$; temperature $\left.=35^{\circ} \mathrm{C}\right)$. 
Table S2. Polymerization of P-I by using additive of HOAc with different ratios ${ }^{\mathrm{a}}$.

\begin{tabular}{ccccccc}
\hline Entry & $\begin{array}{c}\mathrm{HOAc} \\
(\mathrm{eq})\end{array}$ & $\mathrm{Rh}(\mathrm{mol} \%)$ & $M_{\mathrm{n}}{ }^{b}$ & $\bigoplus^{\mathrm{b}}$ & $\begin{array}{c}\text { Conv. } \\
(\%)^{\mathrm{c}}\end{array}$ & $\begin{array}{c}\text { Yield } \\
(\%)^{\mathrm{d}}\end{array}$ \\
\hline 1 & 0 & 2 & $/$ & $/$ & $/$ & $/$ \\
2 & 0.2 & 2 & 3.2 & 1.38 & 56.1 & 31.0 \\
3 & 0.8 & 2 & 11.1 & 1.66 & 89.1 & 59.1 \\
4 & 1.2 & 2 & 12.4 & 1.23 & 92.7 & 76.2 \\
5 & 1.5 & 2 & 12.2 & 1.76 & 90.0 & 61.9 \\
5 & 2 & 2 & 12.3 & 1.67 & 93.6 & 71.4 \\
6 & 4 & 2 & 9.0 & 1.48 & 91.8 & 74.3
\end{tabular}

${ }^{a}$ Polymerization conditions: Toluene was used as solvent and HOAc was 1.2eq. ${ }^{b}$ Determind by DMF size-exclusion chromatography (SEC) calibrated by using polystyrene (PS) standards. ${ }^{c}$ Conversion after Precipitated from $\mathrm{Et}_{2} \mathrm{O} .{ }^{d}$ Isolated yield after purification by $\mathrm{Et}_{2} \mathrm{O}$ from THF.

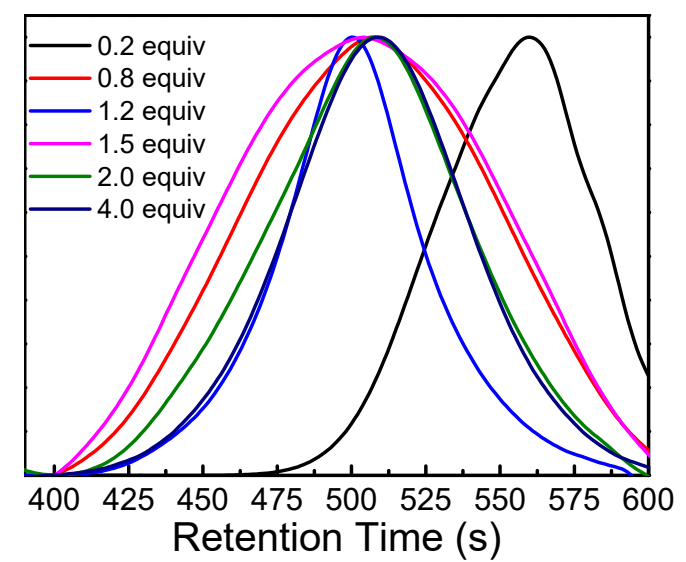

Figure S50. SEC chromatograms of the polymerization of P-I prepared by using additive of HOAc with different ratios (SEC conditions: eluent $=\mathrm{DMF}$; temperature $=35^{\circ} \mathrm{C}$ ).

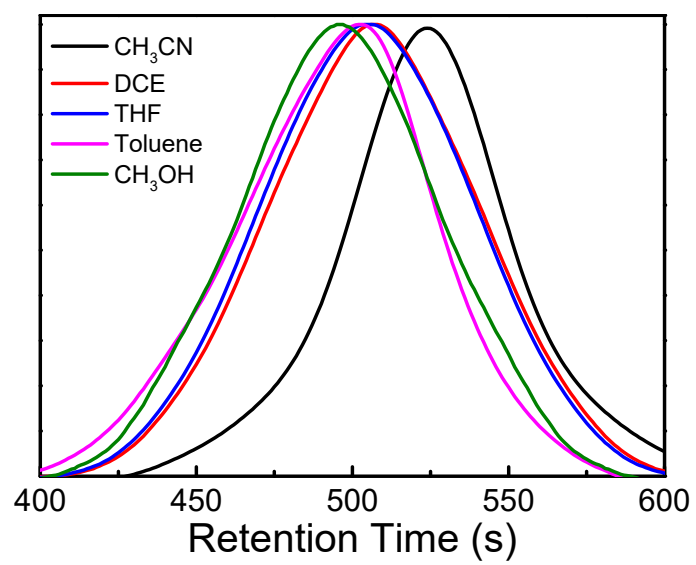

Figure S51. SEC chromatograms of the polymerization of P-II,III prepared by using different solvents $\left(\mathrm{SEC}\right.$ conditions: eluent $=\mathrm{DMF}$; temperature $=35^{\circ} \mathrm{C}$ ). 


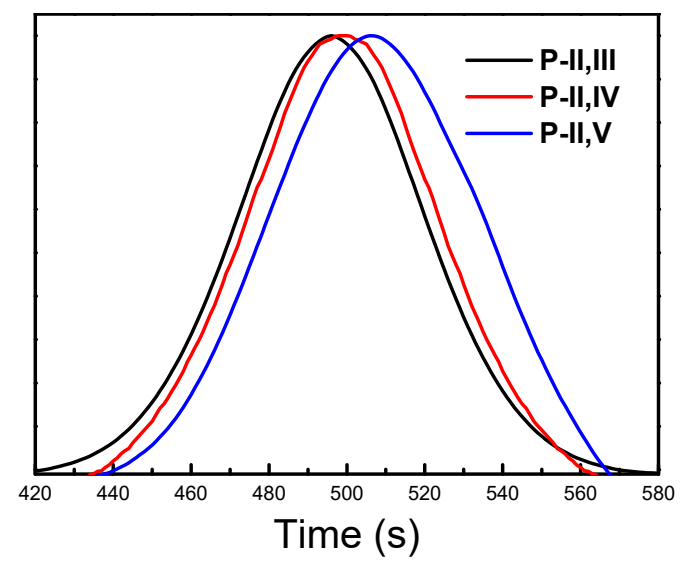

Figure S52. SEC chromatograms of the polymer of P-II,III, P-II,IV, P-II,V prepared by using $\mathrm{CH}_{3} \mathrm{OH}$ as solvent $\left(\mathrm{SEC}\right.$ conditions: eluent $=\mathrm{DMF}$; temperature $\left.=35^{\circ} \mathrm{C}\right)$.

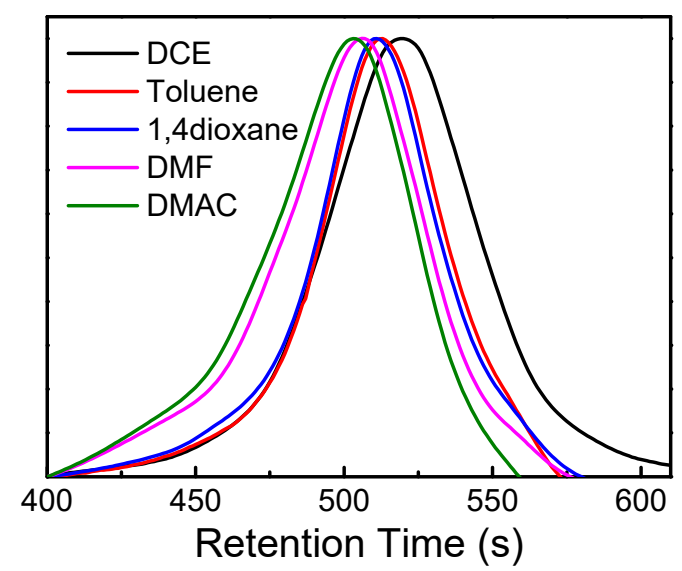

Figure S53. SEC chromatograms of the polymerization of P-II,VI prepared by using different solvents $\left(\right.$ SEC conditions: eluent $=\mathrm{DMF}$; temperature $\left.=35^{\circ} \mathrm{C}\right)$.

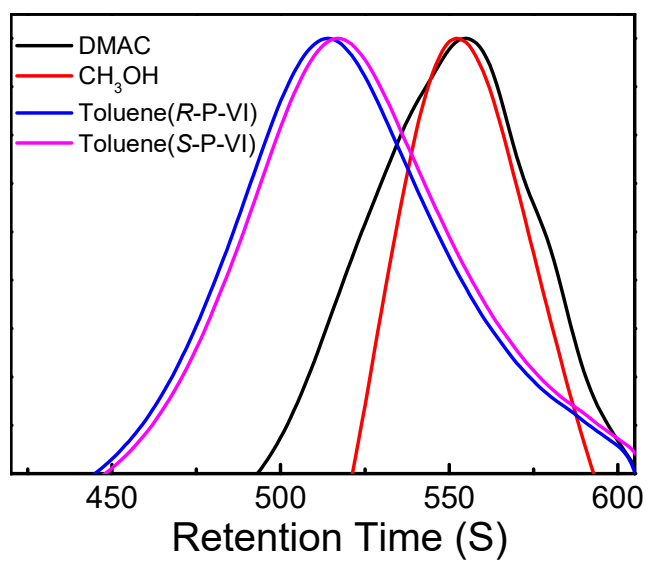

Figure S54. SEC chromatograms of the polymerization of P-II,VII prepared by using different solvents $\left(\right.$ SEC conditions: eluent $=\mathrm{DMF}$; temperature $\left.=35^{\circ} \mathrm{C}\right)$. 


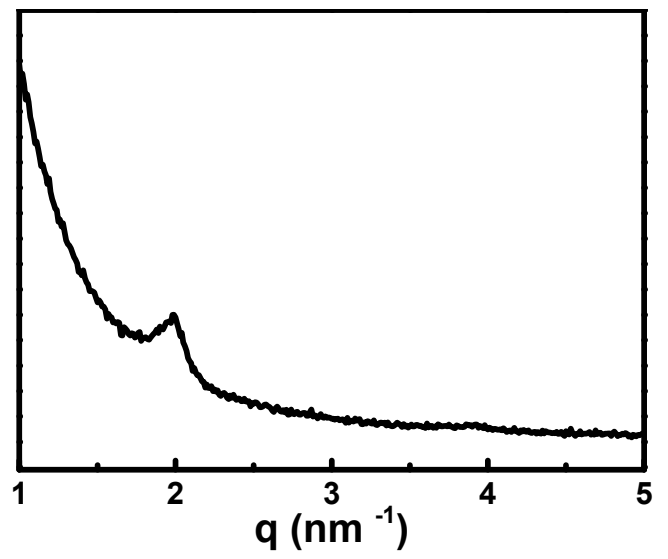

Figure S55. SAXS pattern of P-II,VI upon cooling from $125^{\circ} \mathrm{C}$ (scan time $20 \mathrm{~min}$ ).

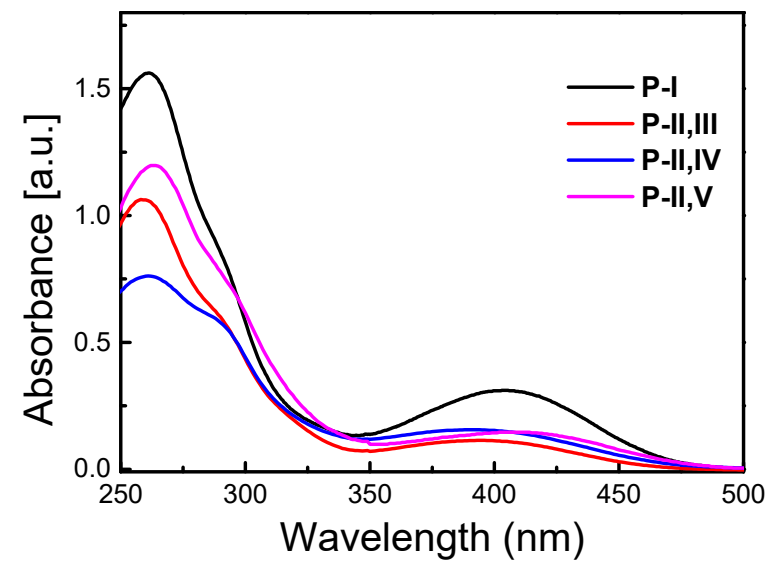

Figure S56. UV-vis absorption spectra of P-I, P-II,III, P-II,IV and P-II,V measured in DMF at $25^{\circ} \mathrm{C}(\mathrm{c}=0.2 \mathrm{~g} / \mathrm{L})$.

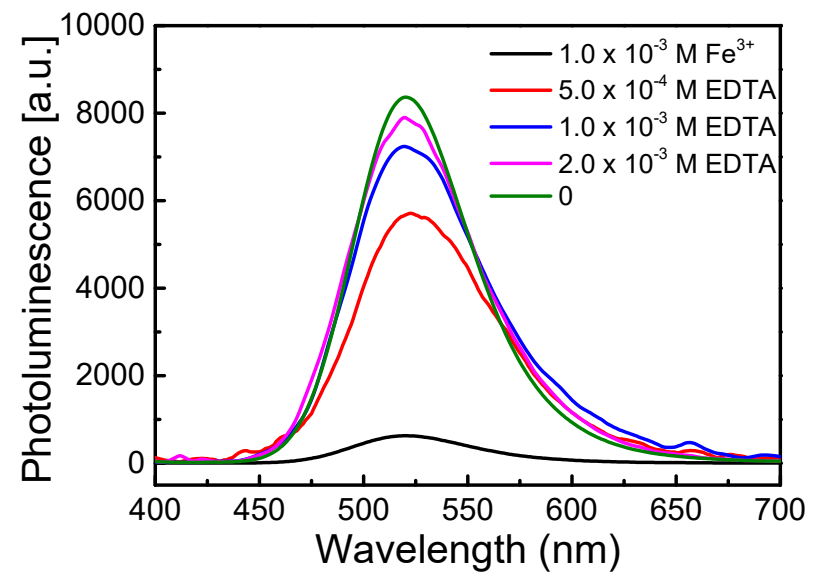

Figure S57. Fluorescence recovery response of P-I to the addition of different concentrations of EDTA. 

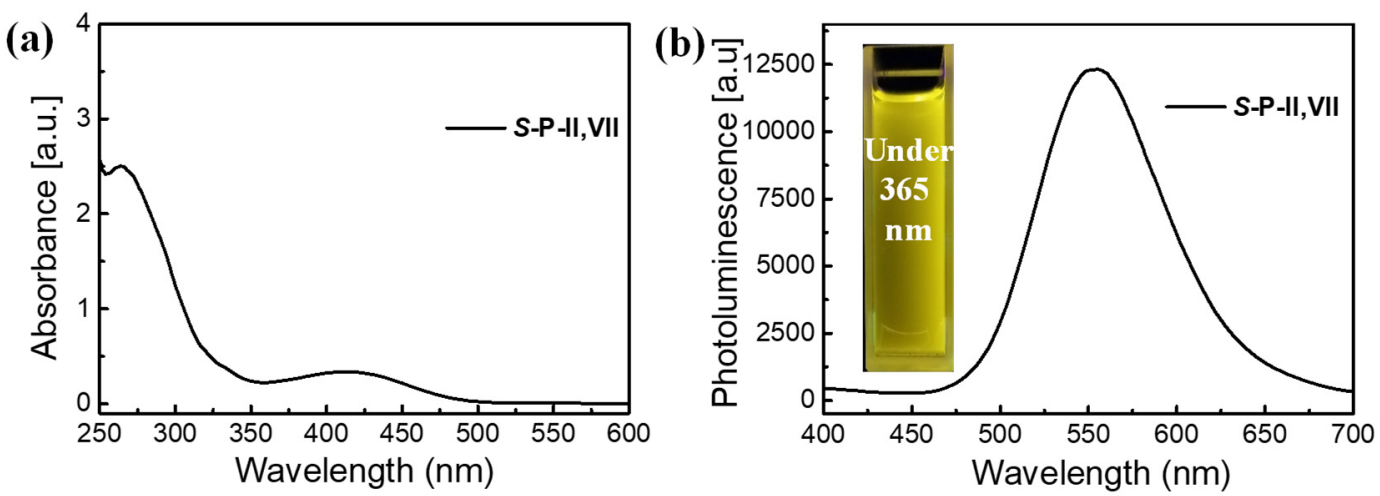

Figure S58. UV-vis absorption spectra of $\boldsymbol{S}$-P-II,VII and PL spectra of spectra of $\boldsymbol{S}$-P-II,VII measured in $\mathrm{CHCl}_{3}$ at $25^{\circ} \mathrm{C}(\mathrm{c}=0.20 \mathrm{~g} / \mathrm{L})$.
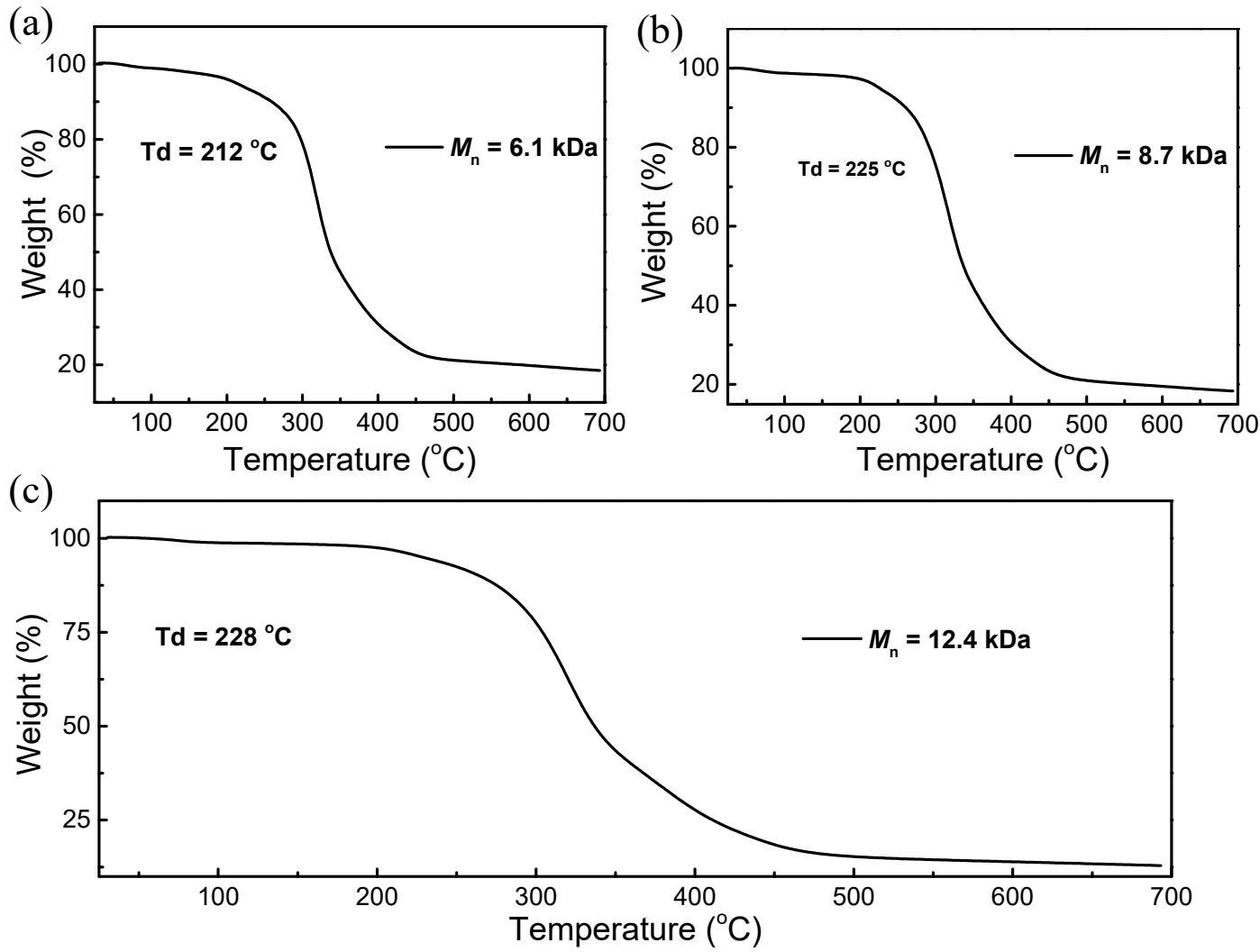

Figure S59. TGA trace of P-I with different molecular weight. 

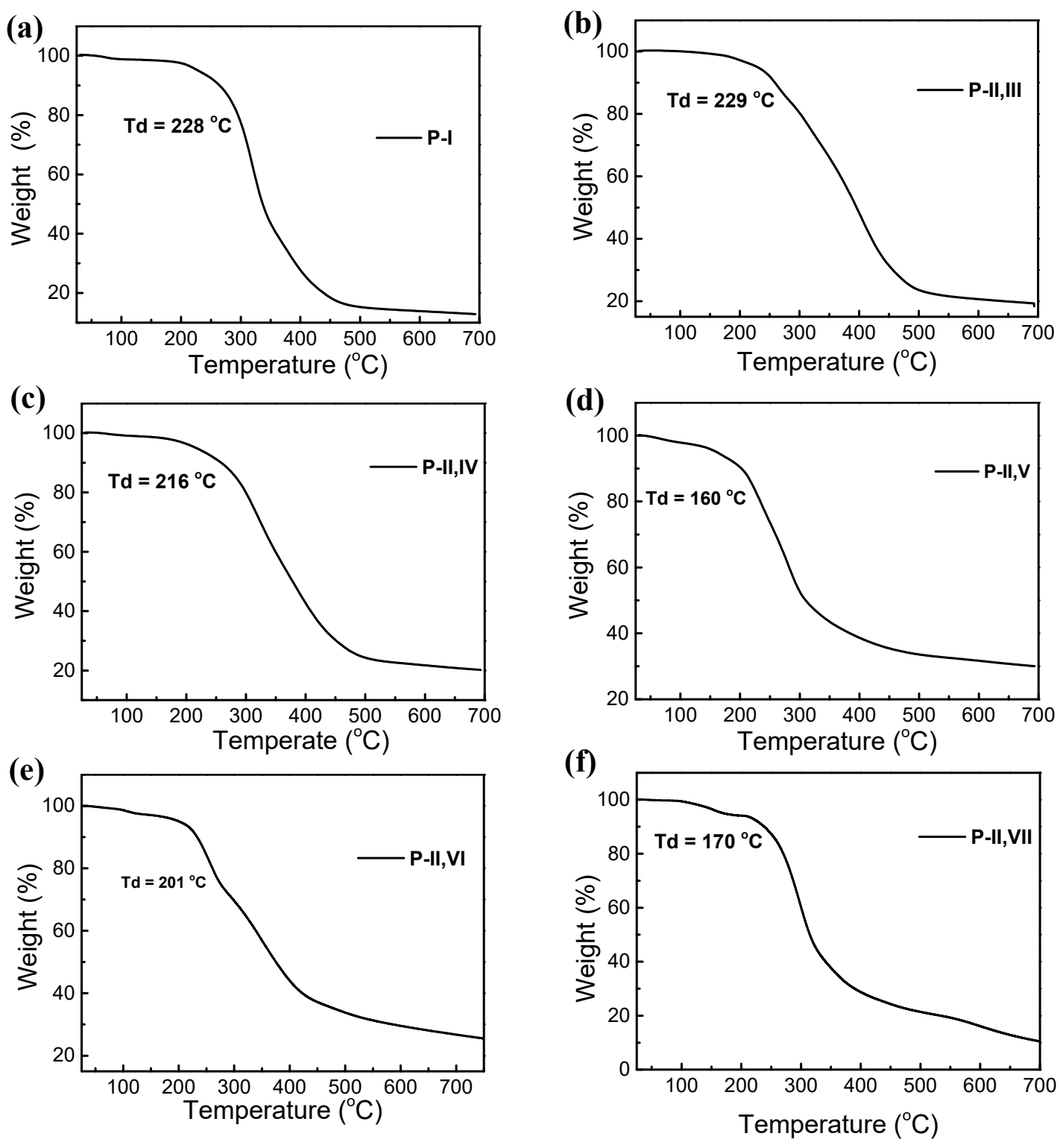

Figure S60. TGA trace of P-I, P-II,III, P-II,IV, P-II,V, P-II,VI, and P-II,VII. 